\title{
Old Physics, New Physics and Colliders *
}

\author{
Ian Hinchliffe \\ Laurence Berkeley Labonatory \\ University of California \\ Berkeley, California 94720
}

Lectures given at the NATO Advanced Study Institute Techniques and Concepts of High Energy Physics IV, July 1986, to be published by Plenum Press, T. Ferbel, ed.

\section{DISCLAIMER}

This report was prepared as an account of wotk sponsored by an agency of the United States Government. Neither the United States Government nor any agency thereor, nor any of their employees, makes any warranty, express or implied, or ascumes any legal liability or responsibility for the accuracy, completeness, of usefulness of any information, apparatus, product, or process discloced, or represents that its use would not infringe privately owned rights. Reference herein to any specific commercial product, process, or service by trade nume, trademark, manufacturer, of otherwise does not necessarily constitute or imply its endorsement, rocommendation, or favoring by the United States Government or any agency theroof. The views and opinions of authors expressed berein do not necessarily state or reflect those of the United States Government or any sgency thereof.

"This work was supported by the Director, Office of Energy Research, Office of High Energy and Nuclear Physics, Division of High Energy Physics of the U.S. Department of Energy under Contract DE-Afen $765 F 00098$. 
OLD PHYSICS, NEW PHYSICS AND COLLIDERS

\author{
I. Hinchliffe \\ Lawrence Berkeley Laboratory \\ University of California \\ Berkeley, Californis 94720
}

\title{
Introduction
}

In these lectures, I shall discuss some topics in the standard model of strong and electroweak interactions and shall then point out some problems in this model and indicate how these problems are attacked in some theoretical models. I shall begin with a discussion of radiative corrections in the Glashow-Weinberg-Salam model, ${ }^{1}$ stressing how these corrections way be measured at LEP and the SLC. I shall then discuss some features of $Q C D$ which are relevant to hadron colliders. This discussion will complement the lectures of Luigi DiLella, ${ }^{2}$ who has shown impressive evidence from the CERN Spp̈S collider for the correctness of QCD. In my discussion of the unsolved problems of the standard model I shall not discuss supersymmetric theories since their theoretical aspects and phenomenological consequences are discussed by other lecturers at this school. ${ }^{3}$ I shall however, discuss some aspects of models in which quarks and leptons are composite particles.

\section{Testing the Weinberg-Salam model.}

The Lagrangian describing the weak and electromagnetic interactions of the quarks and leptons is given by ${ }^{1}$

$$
\begin{aligned}
\mathcal{L}= & -\frac{1}{4} F_{\nu \mu}^{a} F_{a}^{\mu \nu}-\frac{1}{4} G_{\nu \omega} G^{\mu \nu} \\
& +i \bar{\psi}_{L i} \gamma^{\mu} D_{\mu} \psi_{L i} \\
& +\mu^{2}\left(\Phi^{+} \Phi\right)-\lambda\left(\Phi^{+} \Phi\right)^{2} \\
& +\lambda_{\varepsilon h} \bar{\ell}_{L, k} \Phi e_{R, h}+\lambda_{u h l} \bar{q}_{L, k} \Phi^{+} u_{R, l}+\lambda_{d \mu l} \bar{q}_{L, k} \Phi d_{R, l}
\end{aligned}
$$

where

$$
F_{\mu}^{a}=\partial_{\mu} W_{\nu}^{a}-\partial_{\nu} W_{\mu}^{a}+g_{2} \epsilon^{a b c} W_{\mu}^{b} W_{\nu}^{c}
$$

and

$$
G_{\mu \nu}=\partial_{\mu} B_{\nu}-\partial_{\nu} B_{\mu}
$$


are the field strength tensors for the three gauge bosons of $S U(2)_{L}\left(W Y^{a}\right)$ and $U(1)\left(B_{\mu}\right)$, which have coupling constants $g_{2}$ and $g_{1}$. The indices on the fermion fields are generation indices which take values in the rarige $(1,2,3)$.

The left-handed fermions appear in $S U(2)_{L}$ doublets

$$
\psi_{i L}: \quad \ell_{L}=\frac{1}{2}\left(1-\gamma_{5}\right)\left(\begin{array}{l}
\nu \\
e
\end{array}\right), \quad g_{L}=\frac{1}{2}\left(1-\gamma_{5}\right)\left(\begin{array}{l}
u \\
d
\end{array}\right)
$$

which have $U(1)$ charges -1 , and $1 / 3$. The right-handed fermions appear as $S U(2)_{L}$ singlets

$$
\psi_{i R}: \quad e_{R}=\frac{1}{2}\left(1+\gamma_{5}\right) e, \quad u_{R}=\frac{1}{2}\left(1+\gamma_{5}\right) u, \quad d_{R}=\frac{1}{2}\left(1+\gamma_{5}\right) d
$$

with $U(1)$ charges $-2,4 / 3$ and $-2 / 3$, respectively. This pattern is, of course, replcated for the second and third generations which contain the $\mu$ and $\tau$ leptons and the strange, charm, top and bottom quarks. The Higgs doublet $\Phi$ has $U(1)$ charge -1 . The covariant derivatives $D_{\mu}$ are given by

$$
D_{\mu}=\left(\partial_{\mu}-i g_{2} T^{\infty} W_{\mu}^{a}-i g_{1} \frac{y}{2} B_{\mu}\right) \text {. }
$$

Here $y$ is the $U(1)$ charge of the representation on which $D_{\mu}$ acts. For an $S U(2)$ dosblet $T^{a}=\tau^{\circ} / 2$, where $\tau^{\alpha}$ is a Pauli matrix, while for an $S U(2)$ singlet, $T=0$.

This Lagrangian contains seventeen parameters. There are two gauge coupling constants $g_{2}$ and $g_{1}$ describing the interactions of the $S U(2)$ and $U(1)$ gauge theories. Two parameters $\mu$ and $\lambda$ determine the Higgs mass and the interactions of the Higgs field with itself. The remaining parameters are the quark and lepton Yuliawa couplings $\lambda_{i}$. Let us examine the spectrum of physical states in the model.

For $\mu^{2}>0$ the grcund state of the theory is given when the Higgs field $\phi$ has a non-zero vacuum expectation value (VEV):

$$
\langle\Phi\rangle=\left(\begin{array}{c}
0 \\
\frac{v}{\sqrt{2}}
\end{array}\right)
$$

with $v=\left(\mu^{2} / \lambda\right)^{1 / 2}$. This non-zero VEV results in a mass for three of the four gauge bosons. The charged gauge bosons of $S U(2)_{L}$ have mass

$$
M_{W}^{2}=g_{2}^{2} \frac{v^{2}}{4} \text {. }
$$

There is a massless neutral gauge boson, the photon,

$$
A_{\mu}=\sin \theta_{W} W_{\mu}^{3}-\sin \theta_{W} B_{\mu}
$$

and a massive boson

$$
Z_{\mu}=\cos \theta_{W} W_{\mu}^{3}+\cos \theta_{W} B_{\omega}
$$

with mass $M_{Z}^{2}=v^{2}\left(g_{1}^{2}+g_{2}^{2}\right) / 4$. Here the weak mixing angle $\theta_{W}$ is given by

$$
\tan \theta_{w}=\frac{g_{l}}{g_{2}}
$$


Table 1: Couplings of physical particles in the Weinberg-Salam model (unitary gauge). $f$ is a fermion of charge $Q f$ and weak isospin $T_{3}(=+1$ for $u$, $c, t$ quarks and neutrinos, -1 otherwise).

$$
\begin{array}{ll}
W f \bar{f} & \frac{g_{2}}{2 \sqrt{2}} W_{\mu} \bar{f} \gamma^{u}\left(1-\gamma_{5}\right) f \\
Z \bar{f} f & \frac{e}{2 \sin \theta_{W} \cos \theta_{W}} Z^{u} \bar{f} \gamma^{u}\left(v_{f}-a_{f} \gamma_{5}\right) f \\
& V_{f}=\bar{T}_{3}-4 Q \sin ^{2} \theta_{W} \\
& A_{f}=T_{3} \\
H W W & g_{2} M_{W} H W_{\mu}^{+} W_{\mu}^{-} \\
H Z Z & \frac{g_{2}}{2 \cos \theta_{W}} M_{x} Z_{\mu} Z_{\mu} \\
H f \bar{f} & \frac{g_{2} m_{f}}{2 M_{W}} H \overline{f f}
\end{array}
$$

The electric charge of the electron is given by $e=g_{2} \sin \theta_{W}$. The non-zero value of $v$ results in lepton masses

$$
m_{e}=\lambda_{e} \frac{v}{\sqrt{2}} .
$$

The quark masses are more complicated since weak interactions allow transitions between different generations, i.e., $s \rightarrow u+W^{-}$. The Yukawa interactions of the up quarks can be chosen to be diagonal, i.e.,

$$
\lambda_{\mu, j}=\lambda_{L i} \delta_{i, j} .
$$

The masses of the charge $2 / 3$ quarks are then given by

$$
m_{u_{i}}=\lambda_{L_{i}} \frac{v}{\sqrt{2}}
$$

The down quark mass matrix contains seven parameters which are the masses of the $d, s$ and $b$ quarks and the four angles of the Kobayashi-Maskawa mixing matrix. The final parameter is the Higgs mass $m_{H}=\sqrt{2 \lambda} m_{W} / g_{2}$. The theory has a large number of parameters but is able to describe a wealth of experimental data. The most important parameters are $v, g_{1}$ and $g_{2}$ which control the strength of weak and electromagnetic interactions. Most experimental tests of the model do not depend upon quark or lepton masses (or alternatively, on the quark and lepton Yukawa couplings) so that experimental success is more remarkable.

The $W$ and $Z$ bosons couple to quarks, leptons and the remaining physical Higgs boson $H$ with interactions shown in Table 1.

I have so far discussed the model at the tree level, i.e., to lowest order in the coupling constants $g_{1}$ and $g_{2}$. Before discussing tests of the theory, it is worth noting the approximate size of the radiative corrections which can be expected. These corrections will depend upon the fine coupling constant $\alpha=e^{2} /(4 \pi)$. In addition, tests will be made over a large range of momenta. Mornentum transfers can be very small (for example, in Thompson scattering) or very high (for example, the production of a $Z$ or $W$ boson). The gauge interactions produce effects which depend logarithmically 
on these scales. Hence an order of magnitude estimate of radiative corrections will give $\alpha / \pi \log \left(M_{W}^{2} / m_{e}^{2}\right)$. This is of order $5 \%$. Some experiments are already sensitive to corrections of this size; experiments at the $Z^{0}$ resonance performed at $\mathrm{LEP}^{4}$ or the $\mathrm{SLC}^{5}$ will be more sensitive so it is important to discuss radiative corrections in some detail.

I will begin with the radiative corrections in Quantum Electrodynamics. Consider the scattering of two charged particles of mass $M$, at momentum transfer $Q$. To lowest order in $\alpha$, this scattering is described by the exchange of a single photon (Fig. la). If the theory contains a particle of mass $m_{e}$, the effect of this particle can appear at next order in perturbation theory via. the graph of Fig. 1b. The relevant Feynman diagrasn is the one-loop correction to the photon self energy shown in Fig. 2. This graph is given by

$$
\Pi_{\mu \nu}\left(Q^{2}\right)=-e^{2} \int \frac{d^{4} k}{(2 \pi)^{4}} \frac{\operatorname{Tr}\left[\gamma^{\mu}\left(k+m_{e}\right) \gamma^{\nu}\left(k-Q+m_{e}\right)\right]}{\left(k^{2}-m_{e}^{2}\right)\left((k-Q)^{2}-m_{e}^{2}\right)} .
$$

This integral is divergent; we can regulate it by performing the loop integral in $n$ dimensions, i.e. by making the replacement ${ }^{6}$

$$
\frac{d^{4} k}{(2 \pi)^{4}} \rightarrow \frac{d^{n} k}{(2 \pi)^{n}}
$$

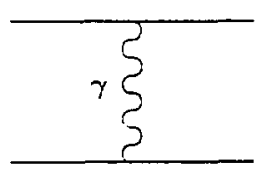

(a)

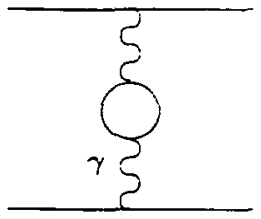

(b)

Figure 1: (a) Feynman diagram showing a contribution to the scattering of two charged particles in QED; (b) A higher order contribution.

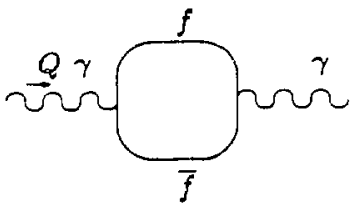

Figure 2: A contribution to the photon self energy at one loop due to a charged fermion of mass $m_{e}$. 
We then have

$$
\begin{aligned}
\Pi_{\mu v}\left(Q^{2}\right) & =\left(Q_{\mu} Q_{\nu}-Q^{2} g_{\mu \nu}\right) \Pi\left(Q^{2}\right) \\
& =\left(Q_{\mu} Q_{\nu}-Q^{2} g_{\mu \nu}\right) \frac{2 i \alpha}{\pi}\left[\frac{2}{4-n}+\log (4 \pi)-\gamma_{E}\right. \\
& \left.-\int_{0}^{1} z(1-z) \log \left(\frac{Q^{2} z(1-z)-m_{e}^{2}}{\mu^{2}}\right) d z+O(n-4)\right] .
\end{aligned}
$$

Here $\gamma_{E}$ is the Euler-Mascheroni constant $\left(\gamma_{E}=0.577\right)$ and $\alpha=e^{2} / 4 \pi$. I have expanded the result around $n=4$ and not written the terms which vanish as $n \rightarrow 4$. The scale $\mu$ has been introduced since, in $n$ dimensions, the interaction of an electron with a photon has the following form

$$
e \mu^{(n-s) / 2} \bar{\psi} \gamma^{\mu} \psi A_{\mu}
$$

The coupling constant $e$ (or $\alpha$ ) then remains dimensioniess in $n$ dimensions. $\mu$ is an arbitrary constant - physics cannot depend upon it.

The scattering of the heavy particles then has an amplitude of the following form

$$
\frac{e^{2}}{Q^{2}}\left[1+i \Pi\left(Q^{2}\right)\right]
$$

In order to make contact with physics, the divergence at $n=4$ must be removed, i.e., the theory must be renormalized. Two renormalization schemes are often used:

(a) Minimal subtraction. ${ }^{7}$ Here the term $\frac{2}{n-4}$ and the attendant constants $\gamma_{E}$ and $\log 4 \pi$ are thrown out. This amounts to defining a renormalized charge

$$
e_{R}^{2}=e^{2}+\frac{e^{2}}{4 \pi^{2}}\left[\frac{1}{(4-n)}+\frac{1}{2} \log 4 \pi-\frac{\gamma_{E}}{2}\right] .
$$

This scheme is very easy to use but it is unphysical; the resulting renormalized coupling constant is not directly related to any physical quantity. This definition is the one normally used in $Q C D$.

(b) Define the renormalized charge so that as $Q^{2} \rightarrow 0$, the scattering amplitude is $e_{R}^{2} / Q^{2}{ }^{8}$ Hence, $e_{R}^{2}=e^{2}[1+i \Pi(0)]$. This definition has the advantage that it can be related directly to a physical quantity, the scattering rate at small momentum transfer. It is the definition used in Quantum Electrodynamics; it corresponds to the value of $\alpha=1 / 137$ measured from Thompson scattering." This definition cannot be used in $Q C D$ since perturbation theory is not reliable as $Q^{2} \rightarrow 0$.

In the limit of large $Q^{2}$, the scattering amplitude has the following form

$$
\frac{\alpha_{R}}{Q^{2}}\left[1+\frac{\alpha_{R}}{3 \pi} \log \left(Q^{2} / m_{c}^{2}\right)\right] \text {. }
$$

I have retained terms of order $\log Q^{2} / \mathrm{m}_{e}^{2}$ only and have used the definition (b) of $\alpha$. We can introduce a running coupling constant $\alpha\left(Q^{2}\right)$ by

$$
\alpha\left(Q^{2}\right)=\alpha\left[1+\frac{\alpha}{3 \pi} \log \left(Q^{2} / m_{\varepsilon}^{2}\right)\right] \text {. }
$$

-The most accurate measurements of $\alpha$ come from the Josephson Junction. ${ }^{9}$ Siure the momentum transiers in this case are extremely small, the value obtained corresponds to the definition discussed here. 
The scattering amplitude is then proportional to $\alpha\left(Q^{2}\right) / Q^{2}$.

In the standard model there are contributions to $\alpha\left(Q^{2}\right)$ from all charged particles. The only one whose mass is not known is the top quark. Each charged particle begins to contribute when $Q^{2}>m_{i}^{2}$. The evolution of $\alpha\left(Q^{2}\right)$ is shown in Fig. 3. At $Q^{2}=M_{W}^{2}$ 10

$$
\alpha_{e m}\left(M_{W}^{2}\right) \sim 1 / 128 .
$$

Having defined the electromagnetic coupling $\alpha_{e m}$, it now remains to specify the other parameters of the Weinberg-Salam model. Apart from the fermion masses and the Kobayashi-Maskawa angles there are three parameters. In the Lagrangian these are $\mu, \lambda$ and one of $g_{1}$ and $g_{2}$. (The other is fixed by $\alpha$ ). At lowest order these parameters can be taken to be $M_{W}, M_{H}$ and $g_{2}$. A renormalized mass is easy to define; it is simply the position of the pole in the particle's propagator which corresponds to its physical mass. $g_{2}$ remains to be defined.

At present the $\mathrm{W}$ mass is not well measured. Consequently, we shall not take it to be one of the fundamental parameters. The Higgs mass must be taken as one parameter. The two remaining ones will be directly related to two accurately measured physical quantities. The muon lifetime is extremely well measured. This can be used to extract the Fermi constant $G_{F} .^{11}$ We need one other quantity. I will take this to be the mass of the $Z$ boson which will be well measured at the SLC or LEP in the near future. The fundamental parameters are therefore: $\alpha_{e m}$, obtained from the Josephson Junction; $G_{F}$, obtained from the muon lifetime; $M_{Z}$ and the Higgs mass $M_{H}$ which does not play a crucial role in the subsequent discussion. This procedure eliminates the need to define the coupling constant $g_{2}$.

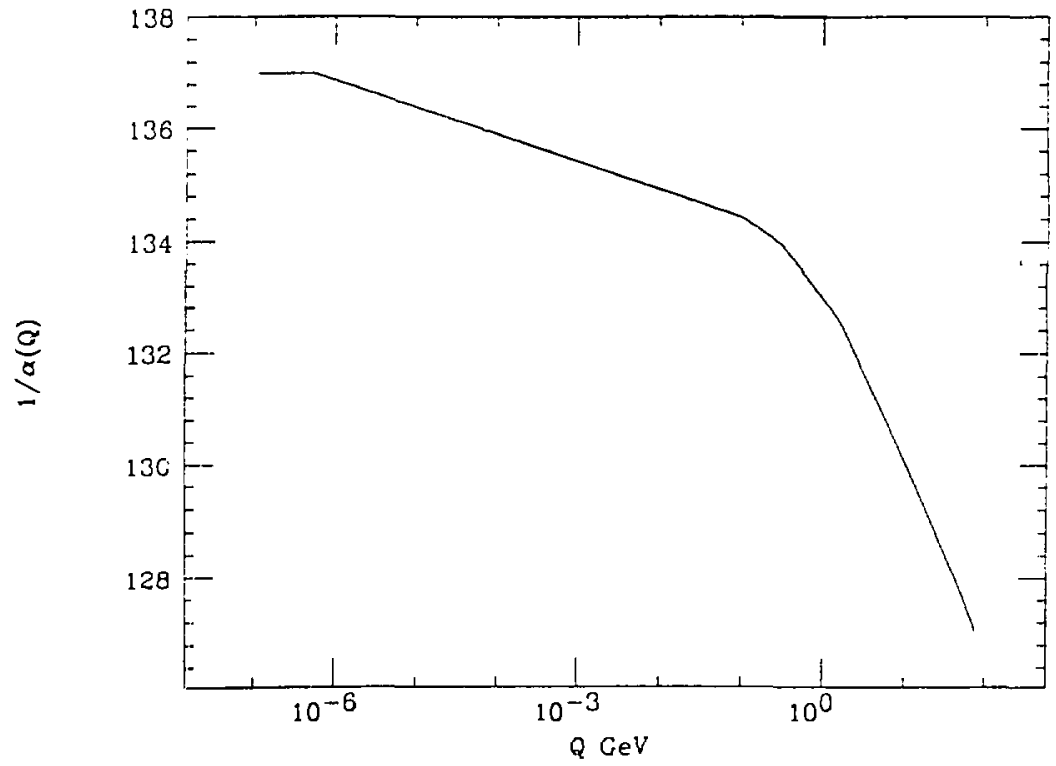

Figure 3: The behavior of $a_{\mathrm{em}}(Q)$ with $Q$, which shows the increase of $\alpha_{\mathrm{em}}(Q)$ due to the known quarks and leptons. 
In lowest order the Fermi constant $G_{F}$ is related to the muon lifetime $\left(\tau_{\mu}\right)$ by

$$
\frac{1}{\tau_{\mu}}=\frac{G_{F}^{2} m_{\mu}^{3}}{192 \pi^{3}}\left[1-\frac{8 m_{e}^{2}}{m_{\mu}^{2}}\right] \text {. }
$$

It is traditional to include higher order $Q E D$ corrections from the graphs of the type shown in Fig. 4. The right-hand side of Eq. (1.15) is modified by a factor ${ }^{11}$

$$
1+\frac{\alpha}{2 \pi}\left[\frac{25}{4}-\pi^{2}\right]\left[1+\frac{2 \alpha}{3 \pi} \log \left(m_{e} / m_{\mu}\right)\right] .
$$

Since $\alpha$ is known, the muon lifetime can be used to extract $G_{F} .^{\dagger}$

Once the $Z$ mass is determined the $W$ mass is predicted ${ }^{12}$ to be

$$
M_{W}=\frac{M_{Z}}{\sqrt{2}}\left[1+\left[1-\frac{4 \pi \alpha}{\sqrt{2} M_{W}^{2} G_{F}(1-\Delta r)}\right]^{1 / 2}\right]^{1 / 2} .
$$

$\Delta r$ includes the effect of radiative corrections, for a top quark mass of $35 \mathrm{GeV}$ and a Higgs mass of $100 \mathrm{GeV}$

$$
\Delta r=0.0696 \pm 0.0020 \text {. }
$$

There is some uncertainty in $\Delta r$. Apart from the unknown top and Higgs masses, the contribution of light quarks in the loops of Fig. 2 is uncertain. This contribution must be gotten from measurements of the cross-section for the process $e^{+} e^{-} \rightarrow$ hadrons since $Q C D$ corrections are not small and cannot be calculated when $Q^{2} \leq 1 \mathrm{GeV}^{2}$.

What has happened to the weak mixing angle $\theta_{W}$ ? In the approach that I have used it is not a fundamental parameter. It can be defined by $\cos \theta_{W} \equiv M_{W} / M_{Z}$. The coupling constant $g_{2}$ can now be defined by $g_{2} \equiv e / \tan \theta_{w}$. In lowest order when $\Delta r$ is zero, $g_{2}$ is related to $G_{F}$ in the usual way

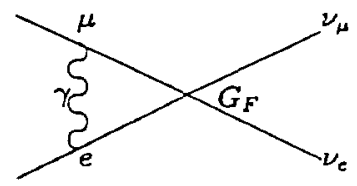

Figure 4: Feynman diagram showing a QED correction to the muon lifetime.

istrictly speaking, a factor of $1+\frac{3 m^{2}}{3 M_{w}^{2}}$ should also be ircluded. This arises since the Fermi interaction is due to the exchange of a $W$ boson which has a propagator of the form

$$
\frac{\left(-g^{\mu \nu}+\frac{q^{2} q^{4}}{M M_{w}^{2}}\right)}{\left(q^{2}-M_{W}^{2}\right)}
$$

If this is expanded as a power series in $q^{2} / M_{W}^{2}$, the leading term is $1 / M_{w}^{2}$. The next order term gives rise to a term proportional to $m_{\mu}^{2} / M_{W}^{4}$. In practice this corsection is irrelevant since its effect is less than the error on $T_{\mu}$. 
Table 2: Values of $M_{Z}$ (or $\sin ^{2} \theta_{w}$ ) extracted from various experiments.

\begin{tabular}{|c|c|c|c|}
\hline Process & $M_{Z}$ & $\sin ^{2} \bar{\theta}_{W}$ & Ref. \\
\hline$e^{+} e^{-} \rightarrow \mu^{+} \mu^{-}$ & $84 \pm 4.8$ & $0.17 \pm 0.02$ & 16 \\
\hline $\left.\begin{array}{l}\nu p \rightarrow \nu p \\
\nu p \rightarrow \bar{p} \rightarrow \nu\end{array}\right\}$ & $91.8 \pm 2.8$ & $0.23 \pm 0.02$ & 17 \\
\hline ed asymmetry & $93.3 \pm 2.1$ & $0.220 \pm 0.014$ & 18 \\
\hline$\nu_{\mu} e \rightarrow \nu_{\mu} e$ & $91.8 \pm 2.8$ & $0.23 \pm 0.02$ & 19 \\
\hline Parity violation in atoms & $98.5 \pm 7.9$ & $0.19 \doteqdot 0.04$ & 20 \\
\hline $\left.\begin{array}{l}\bar{\nu} N \rightarrow \mu X, \bar{\nu} X \\
\nu N \rightarrow \mu X, \nu X\end{array}\right\}$ & $92.4 \pm 0.6$ & $0.226 \pm 0.004$ & 21 \\
\hline
\end{tabular}

$$
G_{F}=\frac{g_{2}^{2}}{4 \sqrt{2} M_{W}^{2}}
$$

There is an alternative renormalization scheme to the one I have described. $e$ and $g_{2}$ can be defined by minimal subtraction ${ }^{13}$ (call these $\bar{e}$ and $\overline{g_{2}}$ ). The weak mixing angle is now defined by

$$
\tan \overline{\theta_{W}}=\bar{e} / \bar{g}_{2} \text {. }
$$

$\bar{\theta}_{W}$ and $\theta_{W}$ are related by $\sin \bar{\theta}_{W}=\sin \theta_{W}+0.006 .{ }^{13}$ In view of the possible confusion I shall not use $\sin \theta_{W}$ in the subsequent discussion.

The present measurements of $M_{Z}$ from UA1 and UA2 has a large error. ${ }^{14}$ Table 2 shows the value of $M_{Z}$ extracted from the anzysis of a large set of low energy experiments. For example, the ratio of cross-sections

$$
\frac{\sigma\left(\nu_{\mu} e \rightarrow \nu_{\mu} e\right)}{\sigma\left(\nu_{\mu} e \rightarrow \nu_{e} \mu\right)}=\frac{7 M_{Z}^{4}-20 M_{Z}^{2} M_{W W}^{2}+16 . M_{W}^{4}}{13 M_{Z}^{4}-28 M_{Z}^{2} M_{W}^{2}+16 M_{W}^{4}}
$$

can be used to extract $M_{Z}$ using the formula of Eq. (1.17) for $M_{W}$. This expression for the ratio of cross-sections is given in lowest order. The errors are too large for radiative corrections to be relevant.

The two types of experiments which have the smallest quoted errors are deep inelastic neutrino scattering ${ }^{21}$ and the asymmetry in polarized electron deuterium scattering. ${ }^{18}$ The ratio of the cross-sections for neutral and charged current deep inelastic neutrino scattering from a nucleon : is given by

$$
\frac{\sigma\left(\nu_{\mu} N \rightarrow \nu_{\mu} X\right)}{\sigma\left(\nu_{\mu} N \rightarrow \mu X\right)}=\frac{\frac{1}{2}-x+\frac{20 x^{2}}{27}+\epsilon\left(\frac{1}{B}-\frac{x}{3}+\frac{3 C_{x}^{2}}{27}\right)}{1+\epsilon / 3} .
$$

In lowest order $x=1-M_{W}^{2} / M_{Z}^{2}$. $\epsilon$ is the ratio of the fraction of nucleon's momentum carried by antiquarks to that carried by quarks. Radiative corrections cause a small shift in $x .^{22}$ This shift depends upon the kinematics of the experiment and is of order 0.005 . The value of $x=0.226 \pm 0.004$ which is quoted has an error which is smaller than this higher order correction.

i $N$ here refers to a target which consists of an equal mixture of protons and neutrons. 


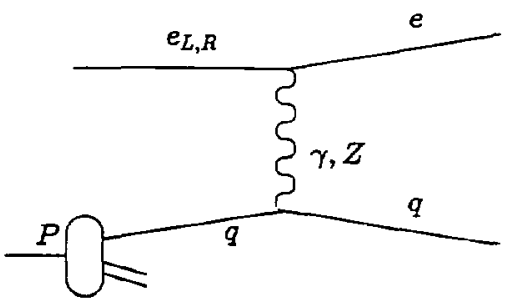

Figure 5: Diagram zelevant for the scattering of polarized electrons from a nuclear target.

There are a number of uncertainties in the value of $x$ extracted from deep inelastic scattering. Firstly, there are QCD corrections to the structure functions. A more important source of uncertainty is that due to the charm quark. There is a contribution to the charged current cross-section from the reaction $\nu+s \rightarrow \mu^{-}+c$. This rate is affected by a threshold factor which depends on the charm quark mass. The neutral current cross-section is affected to a lesser degree since the process $\nu+c \rightarrow \nu+c$ is inhibited due to the small number of charm quarks in the nucleon. If the charm quark mass is allowed to vary from 1.2 to $1.8 \mathrm{GeV}$, there is an uncertainty of order \pm 0.005 in $x .^{21}$ Since the charm quark mass is not determined within this range, I am forced to conclude that this measurement is not sensitive to radiative corrections.

In the case of the scattering of a polarized electron from a deuteron, ${ }^{18}$ one observes the interference between $Z$ and photon exchange (see Fig. 5). The following asymmetry is predicted

$$
\frac{\sigma_{L}-\sigma_{R}}{\sigma_{L}+\sigma_{R}}=Q^{2}\left[a_{1}+a_{2}\left(\frac{1-(1-y)^{2}}{1+(1-y)^{2}}\right)\right],
$$

where $\sigma_{L}\left(\sigma_{R}\right)$ is the cross-section for a left (right) handed electron and

$$
\begin{aligned}
& a_{1}=\frac{-G_{F}}{2 \sqrt{2} \pi \alpha} \frac{9}{10}\left[1-\frac{20 x}{S}\right], \\
& a_{2}=\frac{-G_{F}}{2 \sqrt{2} \pi \alpha} \frac{9}{10}[1-4 x] .
\end{aligned}
$$

The kinematical variable $y$ is the fractional energy loss of the electron: $y=\frac{E_{1}-E_{2}}{E_{1}}$ where $E_{1}\left(E_{2}\right)$ is the incoming (outgoing) electron energy. In this case the higher order corrections compuled for the kinematics of the SLAC ed scattering experiment are $\delta x=0.005 .^{13}$ Again this is comparable to the experimental error. It appears, therefore, that only the next generation of experiments will be able to see higher order corrections.

Table 3 shows the ralues of $M_{W}$ and $M_{Z}$ found by the UA1 and UA2 collaborations. ${ }^{14}$ The predieted value of $M_{Z}$ and $M_{W}$ inferred from the results in Table 2 are shown for comparison. The agreement is remarkable. 
Table 3: Values of $M_{W}$ and $M_{Z}$ measured by the UA1 and UA2 collaborations $^{14}$.

\begin{tabular}{|c|c|c|c|}
\hline . & UA1 & UA2 & $\begin{array}{c}\text { Values }^{15} \text { obtained } \\
\text { using Table } 2\end{array}$ \\
\hline$M_{w}(G e V)$ & $83.5_{-1.0}^{+1.1} \pm 2.7$ & $81.2 \pm 1.1 \div 1.3$ & $\begin{array}{c}81.4 \doteq 0.6 \\
(79.8 \text { without } \\
\text { radiative corr. })\end{array}$ \\
\hline$M_{z}(\mathrm{GeV})$ & $93.0 \pm 1.4 \pm 3$ & $92.5 \pm 1.3 \pm 1.5$ & $\begin{array}{c}92.5 \pm 0.5 \\
(90.2 \text { without } \\
\text { radiative cerr.) }\end{array}$ \\
\hline
\end{tabular}

As I have stressed, most of the radiative corrections are due to known quantities in the standard model such as the coupling of the electron to the gauge bosons. In principle, the accurate measurement of such radiative corrections can give information on the two main uaknown parameters, namely the top quark and Higgs masses. In order to illustrate this, consider the effect of these particles upon the relationship between the $W$ and $Z$ masses (see Eq. (1.17)).

There are contributions to the $W$ and $Z$ self energies from the $t$ and $b$ quarks which are shown in Fig. 6. The evaiuation of these graphs at zero external momentum gives $^{23}$

$$
\begin{aligned}
& \Pi_{W}^{\mu}=-\frac{3 i g^{\mu} g_{2}^{2}}{32 \pi^{2}}\left[\pi_{t}^{2} \log m_{t}^{2} / \mu^{2}+m_{b}^{2} \log m_{b}^{2} / \mu^{2}\right. \\
& \left.+\frac{m_{t}^{2} m_{b}^{2}}{m_{t}^{2}-m_{b}^{2}} \log m_{t}^{2} i m m_{b}^{2}-\frac{1}{2}\left(m_{t}^{2}+m_{b}^{2}\right)\right] \text {, } \\
& \Pi_{Z}^{\mu \nu}=\frac{3 i g^{\mu \nu} g_{z}^{2}}{32 \pi^{2}} \frac{M_{Z_{20}}^{2}}{M_{W_{L 0}}^{2}}\left(m_{t}^{2} \log m_{t}^{2} / \mu^{2}+m_{b}^{2} \log m_{b}^{2} / \mu^{2}\right) \text {. }
\end{aligned}
$$

I have used dimensional regularization and dropped the terms pronortional to $\frac{1}{n-4}$, $\gamma_{E}$ and $\log 4 \pi$. The quanitities $M_{W_{L O}}$ and $M_{Z_{L O}}$ are the lowest order values for the $W$ and $Z$ masses. These contributions cause shifts in the $W$ and $Z$ masses

$$
M_{W, Z}^{z}=M_{W, z, L 0}^{z}\left[1+\frac{\Pi_{W, Z}^{\omega \nu}}{g^{\mu \nu} M_{W, Z, L 0}^{z}}\right] .
$$

These contributions then modify the relationship between the $W$ and $Z$ masses

$$
M_{W}^{2}=M_{W_{L 0}}^{2}\left[1+\frac{3 G_{F}}{8 \sqrt{2} \pi^{2}}\left[\frac{2 m_{t}^{2} m_{b}^{2}}{m_{t}^{2}-m_{b}^{2}} \log \left(\frac{m_{t}^{2}}{m_{b}^{2}}\right)+m_{t}^{2}+m_{b}^{2}\right]\right]
$$

Notice that the $\mu$ dependence (and the terms proportional to $1 /(n-4)$ had I written them) have canceled, i.e., this correction is finite and independent of the renormalization scheme. Notice that the effect of these corrections is to increase $M_{W}$ as the $t$ quark mass rises. Current constraints from low energy experiments and from the measured value the $W$ mass imply $m_{t} \leq 320 \mathrm{GeV}$.

There are also shifts in the $W$ and $Z$ masses which arise from radiative corrections involving the Higgs. ${ }^{24}$ The Feynman diagrams are shown in Fig. 7. The graphs of Fig. $7 \mathrm{c}$ do not contribute to the $\Delta r$ of Eq. (1.17), since these graphs correspond to 


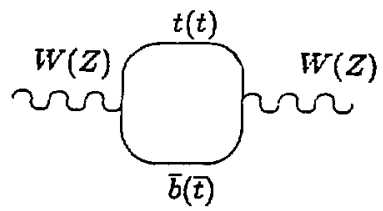

Figure 6: Feynman diagram showing contricutions to the $W$ and $Z$ self energies from the $t$ and $b$ quarks.

a renormalization of the Higgs VEV, whose value does not affect $M_{W} / M_{Z}$. In the limit of large Higgs mass we have

$$
M_{W}^{2}=M_{W L O}^{2}\left(1-\frac{3 G_{F}}{8 \sqrt{2}} M_{Z}^{2}\left(1-\frac{M_{W}^{2}}{M_{Z}^{2}}\right) \log \left(m_{H}^{2} / M_{W}^{2}\right)\right)
$$

The dependence upon $M_{H}$ is rather weak; $M_{W}$ falls slowly as $m_{H}$ is increased. Figure 8 shows the relationship between the $W$ and $Z$ masses for different values of top quark and Higgs masses.

The result that as $m_{t}$ or $m_{H}$ increases the radiative corrections increase, may seem to be contrary to intuition. Consider a theory with a particle of mass $M$. If this theory is probed with energies much less than $M$, then there is a general result known as the decoupling theorem which states that the effect of the heavy particie is proportional to $1 / M^{p}$, where $p$ is some positive number. As $M \rightarrow \infty$, the particle decouples from low energy physics. ${ }^{28}$ Thus, for example, the effects of the $\tau$ lepton on $(g-2)$ of the muon are very small. This theorem is proved under the assumption that the coupling of the particle does not vary with $M$. This is the case for a heavy lepton in $Q E D$ whose coupling to the photon, $\alpha_{t m}$, is independent of $M$.

In the case of the electroweak theory, the couplings cannot be held fixed as $M$ is increased. Recall that the top quark mass is related to the $W$ mass via

$$
m_{t}=\sqrt{2} \frac{\lambda_{t}}{g_{2}} M_{w}
$$

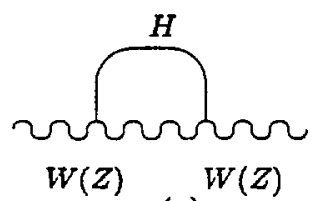

(a)

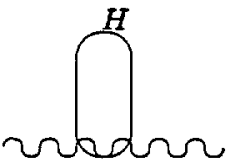

(b)

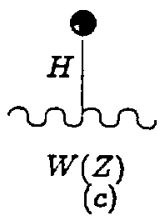

Figure 7: Feynman diagrams showing contributions to the $W$ and $Z$ self energies from the Hizgs boson. 
where $\lambda_{\varepsilon}$ is the Yukawa coupling of the $t$ quark to the Higgs boson. If $M_{W}$ is held fixed and $m_{t}$ is increased, then $\lambda_{t}$ must increase; the top quark interacts more strongly with the Higgs. Clearly, for a sufficiently large $m_{t}, \lambda_{t}$ will be so large that perturbation theory ceases to be reliable. I shall return to this point later. Recall also that the Higgs mass is related to the $W$ mass and the Higgs self interaction $(\lambda)$ by

$$
m_{H}^{2}=2 \lambda M_{W}^{2} / g_{2}^{2}
$$

Again, if $M_{W}$ is held fixed then a large $m_{H}$ implies a large $\lambda$.

The graphs of Figs. 6 and 7 appear to depend only upon the gauge couplings $g_{1}$ and $g_{2}$ and not upon $\lambda_{t}$ and $\lambda$. This is illusory as the following argument will demonstrate. Before the $S U(2) \times U(1)$ symmetry is broken, the theory contains four massless gauge bosons and four scalars (the components of the complex Higgs doublet $\Phi)$. After symmetry breaking, three of the gauge bosons acquire mass. In order to do so they must each gain an additional degree of freedom, their longitudinal polarization states. Three of the scalars supply those degrees of freedom. Hence, "the physical $W$ and $Z$ bosons have some Higgs in them". Couplings of physical W's and $Z$ 's are therefore sensitive to the Higgs Yulawa coupling. ${ }^{5}$ Hence fermions and Higgs bosons of large mass in the standard model do not decouple and can affect the relationship between the $W$ and $Z$ masses.

$A$ t lowest order, there is a relationship (cf. Eqs. (1.3) and (1.4)) between $g_{1}, g_{2}$, $M_{W}$ and $M_{Z}$, viz.,

$$
\frac{M_{W}^{2}}{M_{2}^{2}}=\frac{g_{2}^{2}}{g_{1}^{2}+g_{2}^{2}} .
$$

The form of this relationship is due to the breaking of $S U(2) \times U(1)$ via a Higgs doublet. In models with more complicated Higgs sectors (for example Higgs triplets) this relationship is lost. We can introduce an additional parameter $\rho$ to take account of this possibility

$$
\rho \equiv \frac{\sqrt{2} M_{W}}{M_{Z}\left(1+\left(1-\frac{4 \pi \sigma}{\sqrt{2 G, M_{Z}^{2}(1-\Delta r)}}\right)^{1 / 2}\right)^{1 / 2}}
$$

so that $\rho \equiv 1$ in the standard model. (Note that $\Delta r=0$ in lowest order; in higher orders it should take the value predicted in the minimal model.) If the low enerzy data listed in Table 2 is analyzed with the parameter $\rho$, one gets ${ }^{\text {st }}$

$$
\rho=1.006 \pm 0.008 \text {. }
$$

Since $\rho$ is consistent with 1 it is reasonable to ask if this implies that the Higgs sector of the $S U(2)_{L} \times U(1)$ model is severely constrained.

The part of the $S U(2)_{L} \times U(1)$ Lagrangian describing the self interaction of the Higgs fields, namely

$$
\mu^{2}\left(\Phi^{+} \Phi\right)+\lambda\left(\Phi^{+} \Phi\right)^{4}
$$

has a larger symmetry than $S U(2)_{L} \times U(1)$. One can consider the complex Higgs doublet as having four real components. The interactions of Eq. (1.34) are invariant

\footnotetext{
IThis argument can be seen clearly by writing the theory in 't Hooft-Feynman gauge.
} 
with respect to rotations among these components, i.e., there is an $O(4)=S U(2) \times$ $S U(2)$ symmetry. When the $S U(2)_{L}$ symmetry is broken as one component of the Higgs doublet gets a non-zero VEV, the $O(4)$ symmetry is broken to $O(3)=S U(2)$. It is this $S U(2)$, known as custodial $S U(2),{ }^{28}$ which insures that $\rho=1$. It does this because the resulting mass for the three components $W_{\mu \prime}^{1}, W_{\mu}^{2}$ and $W_{\mu}^{3}$ of the gauge boson multiplet of $S U(2)_{L}$ has the form

$$
M_{W}^{2}\left(W_{\mu}^{1} W_{\mu}^{1}+W_{\mu}^{2} W_{\mu}^{2}+W_{\mu}^{3} W_{\mu}^{3}\right) .
$$

The mixing of $W_{\mu}^{3}$ with $B_{\mu}$ then produces the $Z_{\mu}$ and photon. Any variant of the standard $S U(2) \times U(1)$ model which has a custodiai $S U(2)$ symmetry (e.g., a model with an arbitrary number of Higgs doublets) will predict $\rho=1$. As an example of a model without such a symmetry, suppose that we try to break the $S U(2)_{L} \times U(1)$ symmetry with a mixture of douiviets ar: $d$ triplets. The self interaction of the triplet has an $O(3)$ symmetry which breaks to $O(2)$ when one component acquires a VEV. The residual symmetry of the Higgs sector is then no larger than $O(2)$. There is no custodial $S U(2)$ and $\rho$ is not equal to one. In the case of a model with only triplets $\rho=3 / 2$.

How well can we expect to be able to measure the radiative corrections in the near future? At LEP and the SLC the $Z$ mass can be measured directly. The error on $M_{Z}$ is controlled by the accuracy with which the beam energy can be measured. An error $\delta M_{Z}=50 \mathrm{MeV}$ would seem to be reasonable.,5 The $W$ mass can be measured at LEP from the reaction $e^{+} e^{--} \rightarrow W^{+} W^{-}$. The shape of the cross-section and energy distribution of leptons from the decays $W \rightarrow$ e $\nu$ can be used. Studies ${ }^{27}$ indicate that an error $\delta M_{W} \simeq 100 \mathrm{MeV}$ should be achievable.

Since it will be several years before the $W$ mass can be measured at LEP, it is reasonable to ask how weil one can measure the $W$ mass at hadron colliders. The $W$ mass must be inferred from the transverse momentum distribution of the leptons from the decay $W \rightarrow$ en. As can be seen from Table 3, the current errors on the $W$ and $Z$ mass are large. Part of the error on the $W$ mass is a systematic error arising from calibration. This error can largely be eliminated once the $Z$ mass has been measured in $e^{+} e^{-}$annihilation since leptons from $Z$ decay have known transverse momentum and can be used as a calibration. The remaining error is a statistical one which can be reduced as more W's and $Z$ 's are produced. The proposal ${ }^{28}$ for the $D 0$ detector at the Tevatron claims that an error $\delta M_{W} \approx 100 \mathrm{MeV}$ can be obtained. A value of $\delta M_{W} \approx 300 \mathrm{MeV}$ appears to be achievable in the near future. Such an error implies a sensitivity to $t$ quark masses greater than $90 \mathrm{GeV}$ (see Fig. 8).

Other tests of the electroweak theory can arise from measurements of asymmetries at LEP of the SLC. ${ }^{29}$ I shall concentrate my discussion on those asymmetries measured at the $Z^{\circ}$ resonance where the event rates are large and a good statistical sample can be obtained. The total cross-section for $e^{+} e^{-} \rightarrow Z \rightarrow$ all is approximately $40 n b$.

Measurements of asymmetries can have much smaller :rrors than measurements of rates themselves. This is because certain systematic errors, for exariple in the luminosity measurement, will cancel out. The first asymmetry that I will discuss is the forward-backward asymmetry for the process $e^{+} e^{-} \rightarrow Z \rightarrow f \bar{f}$, where $f$ is a fermion 


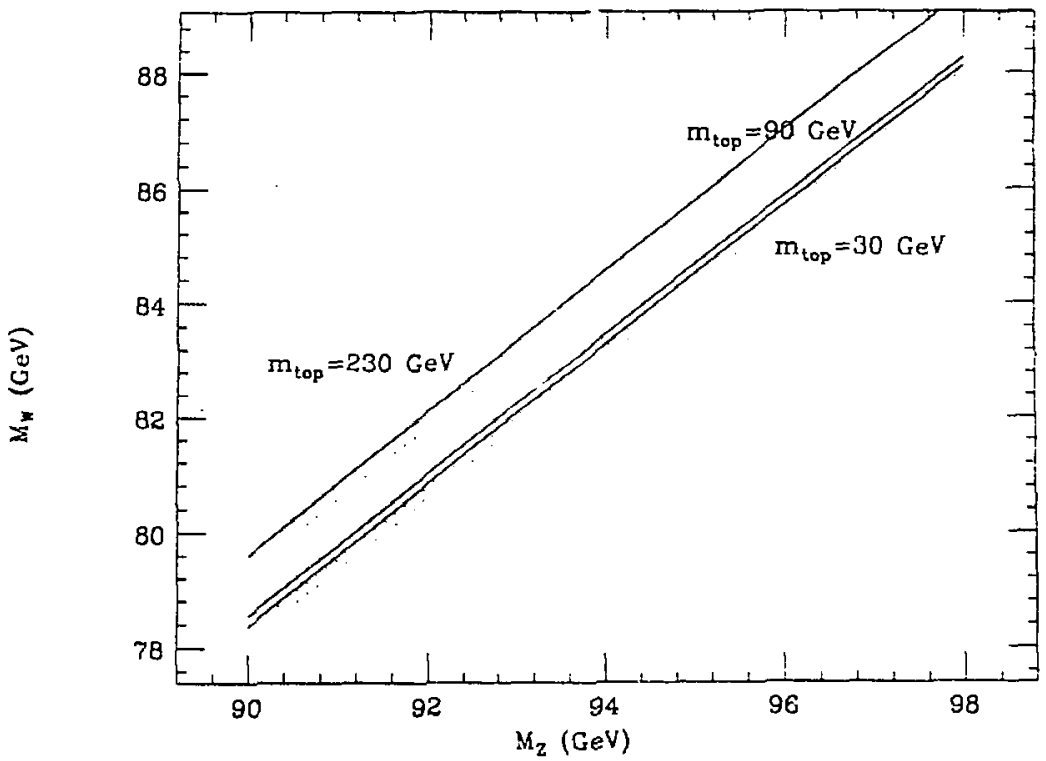

Figure 8: The dependence of $M_{W}$ upon $M_{Z}$ for several choices of $m_{t}$ The solid lines are for $m_{H}=10 \mathrm{GeV}$ and the dotted for $m_{H}=$ $1000 \mathrm{GeV}$.

$$
A_{F B}=\frac{\int(\sigma(f, \theta)-\sigma(\bar{f}, \theta)) d(\cos \theta)}{\int(\sigma(f, \theta)+\sigma(\bar{f}, \theta)) d(\cos \theta)} .
$$

Here $\sigma(f, \theta) \quad(\sigma(\bar{f}, \theta))$ is the cross-section for the production of $f(\bar{f})$ at angle $\theta$ to the $e^{-}$beam. In lowest order this asymmetry is given by

$$
A_{F B}=3 \frac{v_{e} v_{f} a_{e} a_{f}}{\left(v_{e}^{2}+a_{e}^{2}\right)\left(v_{f}^{2}+a_{f}^{2}\right)} .
$$

The quantities $v_{i}$ and $a_{i}$ are given in Table 1. In order to measure this asymmetry it is necessary to distinguish the $f$ from the $\bar{f}$. This is not possible if $f$ is an up, down or strange quark. It may be possible for $c$ and $b$ quarks where the semileptonic decay produces a leptori whose charge is correlated with that of the quark. Clearly, the cleanest final state occurs if $f$ is a muon. I will specialize my discussion to this case. Figure $9^{2 B}$ shows $A_{F B}$ as a function of the $Z$ mass. Three curves are shown: the lowest order prediction and the value radiatively corrected for $m_{t}=30 \mathrm{GeV}$ with $m_{H}=10 \mathrm{GeV}$ and $m_{H}=1 \mathrm{TeV}$.

How well can $A_{F B}$ be measured? At an $e^{+} e^{-}$luminosity of $10^{31} \mathrm{~cm}^{-2} \mathrm{sec}^{-1}$, there are approximately $1000 \mu^{+} \mu^{-}$events per day. If we neglect systematic errors, a LEP experiment with an exposure of 200 days can achieve $\delta A_{F B} \approx 0.002$. An experiment at SLC, with its expecied lower luminosity, is likely to have an error which is at least three times larger. 


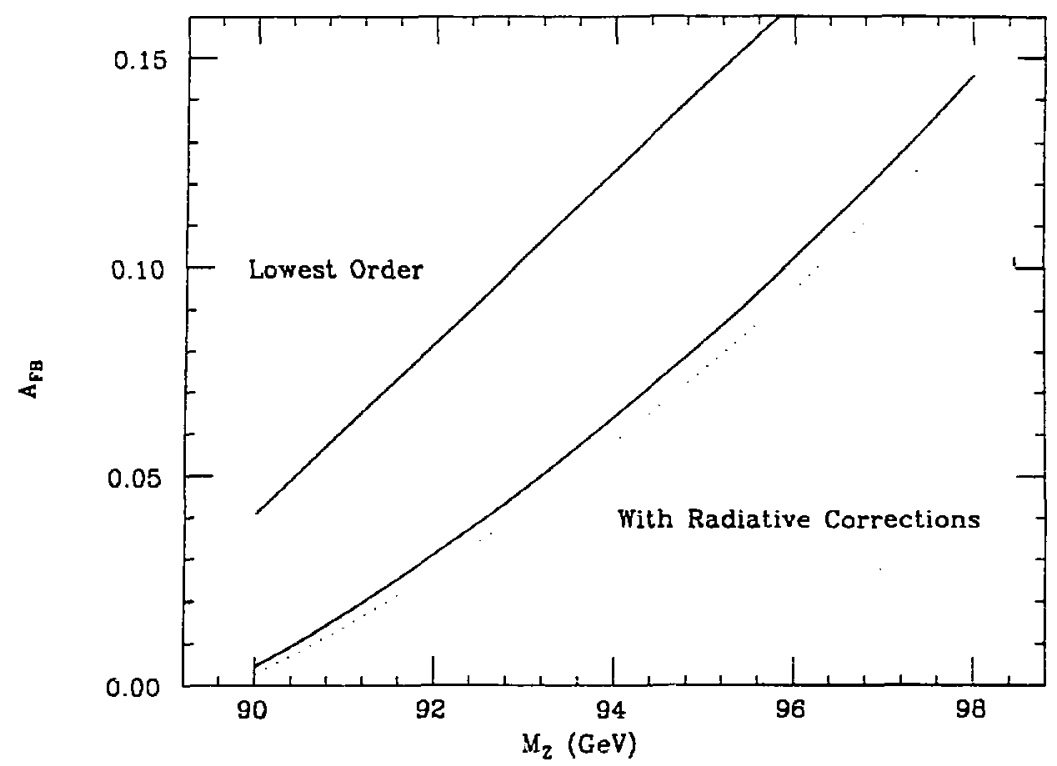

Figure 9: The dependence of $A_{F B}$ for the process $\mathrm{e}^{+} \mathrm{e}^{-} \rightarrow Z \rightarrow \mu^{+} \mu^{-}$for unpolarized $e^{+} e^{-}$beams as a function of $M_{z}$. The solid lines are for $m_{H}=10 \mathrm{GeV}$ and the dotted for $m_{H}=1000 \mathrm{GeV}$.

It is clear that, with an accurary of this order, an experiment can detect the difference between a calculation in lowest order and one including radiative corrections. Most of these radiative corrections arise from known physics, such as the coupling of the electron to the $W, Z$ and photon. Figure 10 shows the contribution to $A_{F B}$ from an additional quark doublet. As in the case of corrections to the $W$ mass, the correction becomes large in the region of large quark masses. The curves are shown as a function of the mass of the charge $2 / 3$ quark for a fixed value of the ratio of the quark masses in the doublet. I have indicated regions on the figure which can ve excluded by other measurements. If the charge $1 / 3$ quark has mass less than $M_{Z} / 2$ it will be observed directly in $Z$ decay so that the region above the dot-dashed line is probed. If the $W$ mass is within $300 \mathrm{MeV}$ of its predicted value, the region above the dotted line will be excluded; an error of $100 \mathrm{MeV}$ rules out the region above the dashed line. I have indicated a $\pm 2 \sigma$ error bar for the LEP scenario discussed above. I an forced to conclude that $A_{F B}$ is not a sufficiently sensitive quantity to be used as a probe of new physics.

Figure 11 shows the contribution to $A_{F B}$ from a doublet of squarks stch as will occur in a supersymmetric version of the standard model. Notice that if the up and down squarks are degenerate the contribution to $A_{F B}$ is zero at large squark masses. This is an example of decoupling since the squarks can have equal, non-zero, masses even if the $S U(2) \times U(1)$ symmetry is unbroken and a large degenerate mass does not imply a large Yukawa coupling. If the ratio of the squark masses is large, then there is no decoupling since the splitting violates $S U(2)$ symmetrv and must arise from the vacuum expectation value of Higgs fields. 
Table 4: The error estimated on the left-right asymmetry as a function of the number of produced $Z^{0}$ 's $(N)$ and the accuracy of the measurement of the electron polarization $(\Delta P / P)^{30}$.

\begin{tabular}{cccc}
\hline$\Delta P / P$ & $N=10^{4}$ & $N=10^{5}$ & $N=10^{6}$ \\
\hline $5 \%$ & 0.025 & 0.013 & 0.010 \\
\hline $3 \%$ & 0.023 & 0.009 & 0.006 \\
\hline $1 \%$ & 0.022 & 0.007 & 0.003 \\
\hline
\end{tabular}

If the polarization of the outgoing fermion $f$ can be measured, then a polarization asymmetry $A_{\text {pox }}$ can be determined

$$
A_{\text {pol }}=\frac{\sigma(h=1)-\sigma(h=-1)}{\sigma(h=1)+\sigma(h=-1)}
$$

where $\sigma(h)$ is the cross-section for the production of $f$ with helicity $h$. In lowest order $A_{\text {pod }}$ is given by

$$
A_{p o l}=\frac{2 v_{f} a_{f}}{\left(v_{f}^{2}+a_{f}^{2}\right)} .
$$

The only particle whose polarization can be measured is the tau lepton. In the decay $\tau \rightarrow \pi \nu$, the momentum spectrum of the $\pi$ is sensitive to the tau helicity

$$
\frac{d W}{d X_{\pi}}=1-h\left(2 X_{\pi}-1\right)
$$

where $X_{\pi}=2 E_{\pi} / \sqrt{3}$. The branching ratio $\tau \rightarrow \pi \nu$ is only $10 \%$ or so. The error on $A_{\text {pod }}$ from such a measurement is unlikely to be small enough for one to be sensitive to new physics.

If the electron or positron beam can be polarized then one can measure

$$
A_{L R}=\frac{\sigma(L)-\sigma(R)}{\sigma(L)+\sigma(R)}
$$

Here $\sigma(L)(\sigma(R))$ is the cross-section for producing a $Z$ from a left (right) polarized electron and an unpolarized positron. $A_{L R}$ is given by

$$
A_{L R}=\frac{2 v_{e} a_{e}}{\left(v_{e}^{2}+a_{e}^{2}\right)} \text {. }
$$

Since there are no plans for polarization at LEP, I will discuss the SLC where a polarized electron source is under construction. ${ }^{30}$ Since the total 2 production rate is used in the measurement of $A_{L R}$, the statistical errors are smaller. There is a systematic error due to the measurement error on the polarization of the electron beam $(\Delta p / p)$. Table 4, extracted from the proposal to measure $A_{L R}$, shows the error on $A_{L R}$ as a function of $\Delta p / p$ and of the number of produced $Z^{0}$ 's. For orientation, at a luminosity of $10^{30} \mathrm{~cm}^{-2} \mathrm{sec}^{-1}$ it takes approximately one year of running to produce $10^{0} Z^{0}$ s. The value of $A_{L R}$ is shown in Fig. 12 as a function of $M_{Z}$ in three different scenarios, all of which are consistent with current data. In order to establish a $3 \sigma$ effect which discriminates between $m_{t}=30 \mathrm{GeV}$ and $m_{\mathrm{t}}=180 \mathrm{GeV}$, it will be 


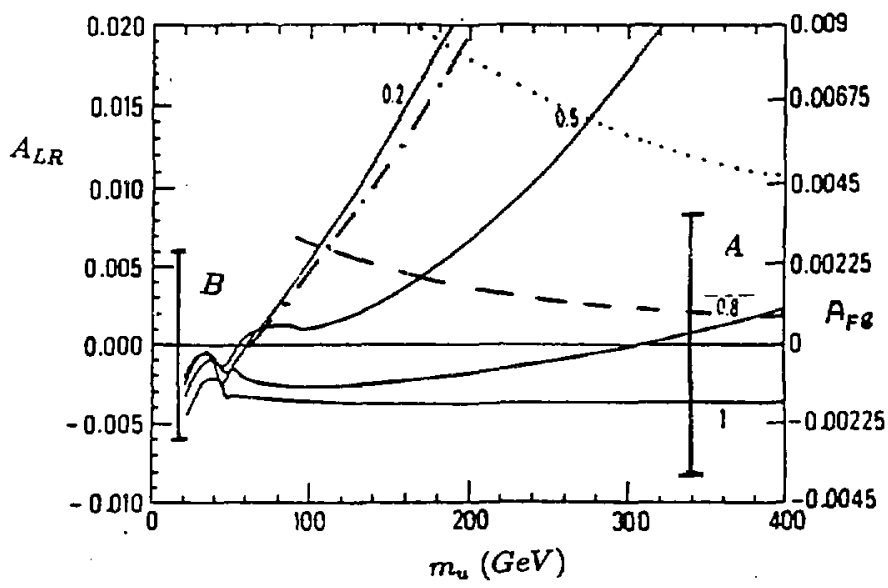

Figure 10: The contribution to $A_{F B}$ and to $A_{E R}$ from the presence of an additional quark doublet as a function of $m_{u}$, the mass of the charge $2 / 3$ member of the doublet. The curves are labelled by the ratio $m_{u} / m_{d}$, where $m_{d}$ is the mass of the charge $1 / 3$ member. The region above the dot-dashed line can be probed directly since $m_{d}$ is low enough for the $Z$ to decay to $d \bar{d}$. If $M_{W}$ is within $300(100) \mathrm{MeV}$ of it predicted value, the region above the dotted (dashed) line is excluded. The error bar $A$ applies to $A_{F B}$ and $B$ to $A_{L R}$ (see text).

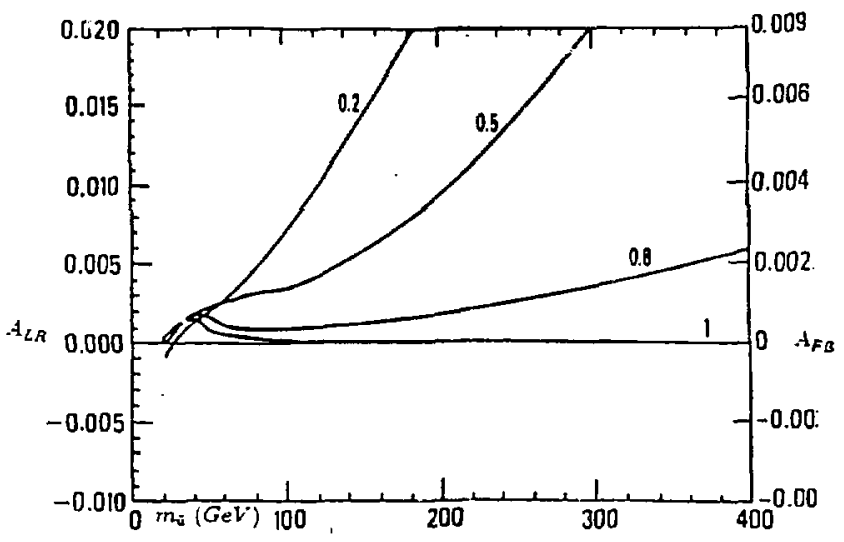

Figure 11: The contribution to $A_{F B}$ from a squark doublei as a function of the up squark mass for fixed ratios of the up to down squark masses. 


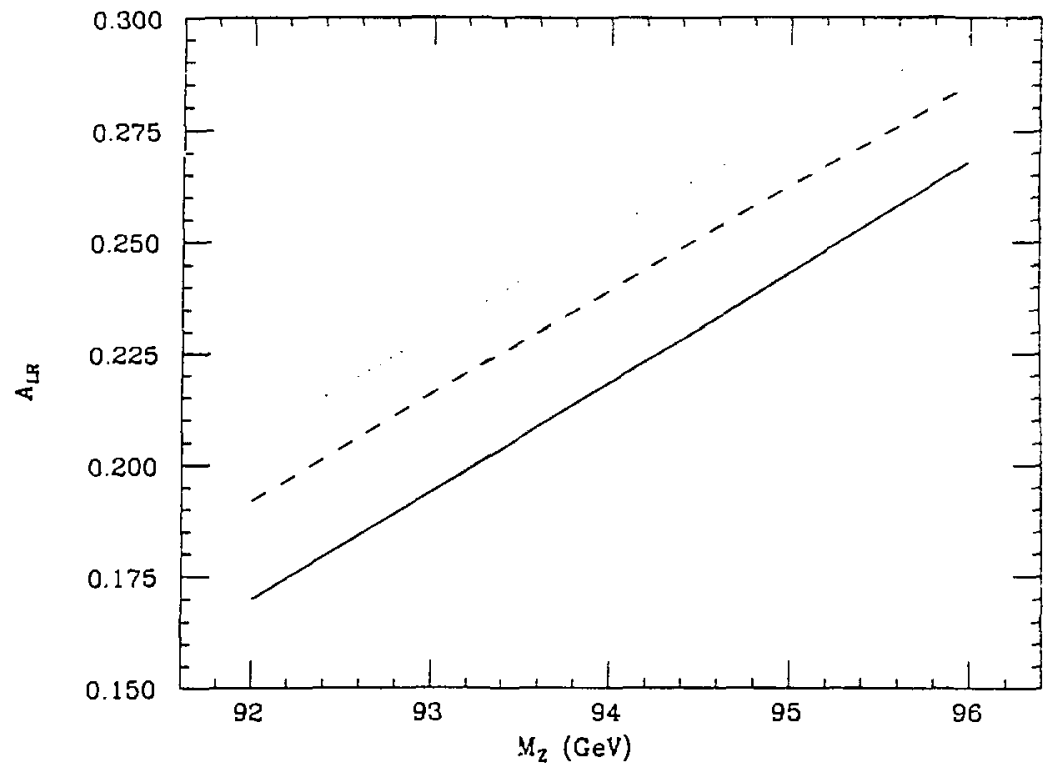

Figure 12: The quantity $A_{L R}$ as a function of $M_{Z}$. The solid line has $m_{\mathrm{t}}=30 \mathrm{GeV}$ and $m_{H}=100 \mathrm{GeV}$. The dashed line has $m_{t}=180 \mathrm{GeV}$ and $m_{H}=100 \mathrm{GeV}$. The dotted line has $m_{t}=30 \mathrm{GeV}$ and a modified Higgs sector such that $p=1.01$.

necessary to measure the polarization to better than $2 \%$ and have more than $10^{5}$ produced $Z^{0}$ 's. Figure 10 shows $\delta A_{L R}$ due to a new quark doublet. I have indicated a $\pm 2 \sigma$ error bar corresponding to $\Delta p / p=1 \%$ and to $10^{6}$ produced $Z^{0}$ s. It may be somewhat easier to establish an effect than in the case of the forward-backward asymmetry.

Before leaving this subject, I would like to comment briefly spon the effect of a more radical modification of the standard model. ${ }^{31}$ The recent upsurge in string theory has provided a motivation for considering models where the gauge group is extended. I shall discuss one particular example where the low energy group is $S U(3) \times S U(2)_{2} \times U(1)_{y} \times U(1)_{y}$. In this model the charged current structure is unaffected but there are changes in the neutral current due to the presence of an additional neutral gauge boson associated with the group $U(1)_{y^{\prime}}$. I shall assume that the coupling constant $g^{\prime}$ of this group is equal to $g_{1}$, a choice supported by these string motivations. If the electric charge operator $Q$ has the same value as in the standard model, viz., $Q=T_{3}+y / 2$, then the photon will be the same linear combination of $B$ and $W_{3}$ as in the standard model. The two massive neutral gauge bosons will be linear combinations of the standard model $Z$ and $B^{\prime}$, the gauge boson of the $U(1)_{y^{\prime}}$ group.

The mass matrix of the neutral bosons will depend upon the structure of the Higgs sector and will have the following form 


\section{$-19-$}

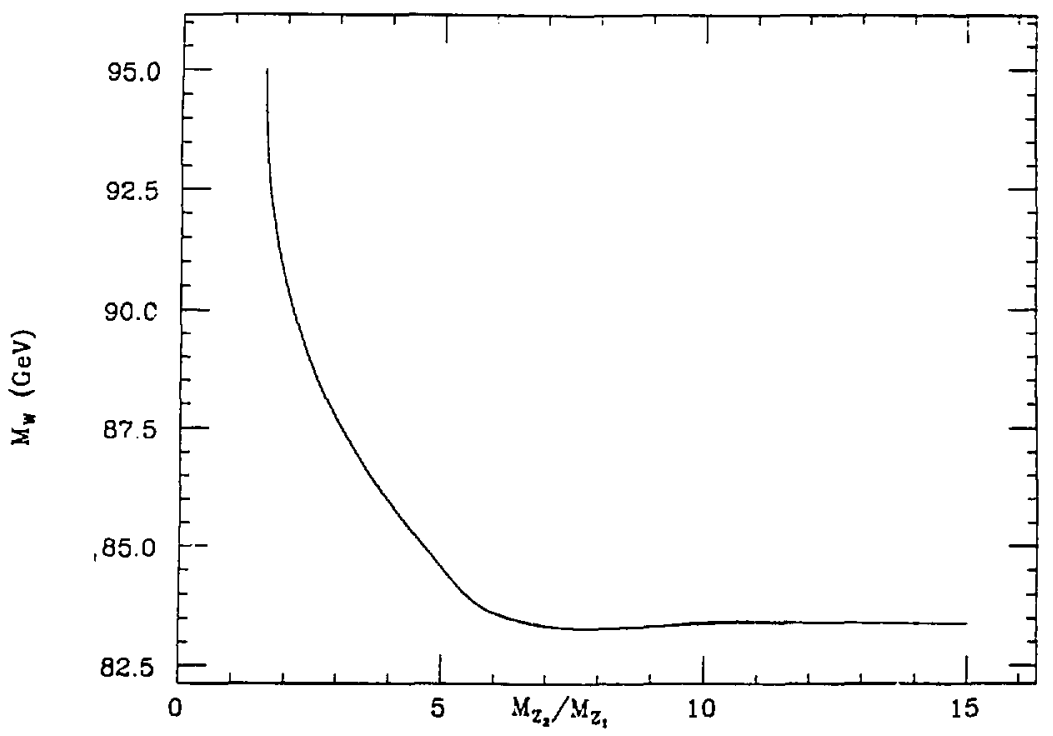

Figure 13: The dependence of $M_{W}$ upon $M_{Z_{3}} / M_{Z_{1}}$ in the model based on $S U(2) \times U(1)_{y} \times U(1)_{:}$for $M_{Z_{1}}=94 \mathrm{GeV}$ and $\rho_{y^{\prime}}=1 / 3$.

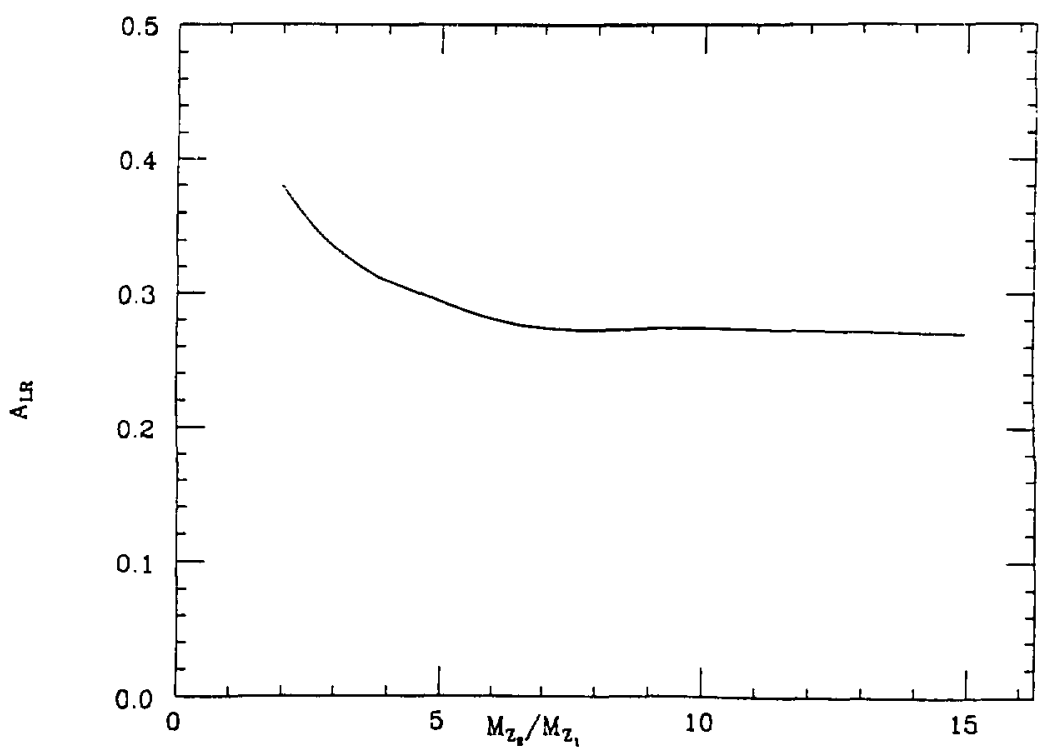

Figure 14: The left-right asymmetry $A_{L R}$ as a function of $M_{Z_{2}}$ for $\rho_{y^{\prime}}=$ $1 / 3$ in the $S U(2) \times U(1)_{y} \times U(1)_{y}$ model. The asymmetry is evaluated at $\sqrt{\mathrm{s}}=M_{Z_{1}}$ which is assumed to be $94 \mathrm{GeV}$. 


$$
|M|^{2}=\left|\begin{array}{ll}
\left(g_{2}^{2}+g_{1}^{2}\right) A & \left(g_{2}^{2}+g_{1}^{2}\right)^{1 / 2} g_{1} B \\
\left(g_{1}^{2}+g_{2}^{2}\right)^{1 / 2} g_{1} B & g C
\end{array}\right|
$$

where

$$
\begin{aligned}
& A=\sum_{i}\left\langle\phi_{i} T_{3}^{2} \phi_{i}\right\rangle, \\
& B=\sum_{i}\left\langle\phi_{i} \frac{T_{3}}{2} y_{i}^{\prime} \phi_{i}\right\rangle, \\
& C=\sum_{i}\left\langle\phi_{i} \frac{y_{i}^{2}}{4} \phi_{i}\right\rangle .
\end{aligned}
$$

Here the sum $i$ runs over Higgs representations $\phi_{i}$ with $U(1)_{V^{\prime}}$ charge $y_{i}^{\prime}-T^{3}$ is the neutral generator of $S U(2)_{L}$. In the case of the standard model $y^{i}=0$ and $A=v^{2} / 4$. The eigenvalues are $M_{Z_{1}}$ and $M_{Z_{2}}$. If we assume that there are only doublets under $S U(2)_{L}$ and singlets under $S U(2)_{L} \times U(1)_{\nu}$, then in the limit $y^{\prime}=0$ we will recover the standard model with $\rho=1$ and a non-minimal Higgs sector.

The model now has five parameters. The three of the standard model, $\alpha, G_{F}$ and $M_{Z_{1}}$ together with $M_{Z_{2}}$ and a parameter describing the Higgs structure analogous to $\rho, \rho^{\prime}=B / A$. The $W$ mass is predicted in terms of these parameters; it is shown in Fig. 13. A measurement of $M_{w}$ to an accuracy of $300 \mathrm{MeV}$ is sensitive to the mass of the second massive neutral gauge boson provided that it is lighter than $300 \mathrm{GeV}$.

The left-right asymmetry measured on the $Z_{1}$ resonance is shown in Figure 14. Again it would appear that the best experiments are sensitive to $M_{z_{3}} \leqslant 300 \mathrm{GeV}$. This value is clase to that which can be observed directly at the Tevatron collider from the production of the $Z_{2}$ boson followed by its decay into $e^{+} e^{-}$or $\mu^{+} \mu^{-}$.

What can we conclude about the potential of measurements of radiative corrections? As wa have seen it will be very difficult for an experiment to be sensitive to new physics; the best hopes seem to lie with a precise determization of the $W$ and $Z$ masses and with the left-right asymmetry. It is very important to emphasize that if an effect is seen in measurements of radiative corrections it may be very difficult to discern its origin. Only the direct observation of new particles can resolve ambiguities.

\section{Where is the Higgs?}

There is very little experimental information about the Higgs sector of the $S U(2) \times$ $U(1)$ model other than that it must have a custodial $S U(2)$ symmetry so that $\rho$ is equal to one at tree level. Do we know anything from theoretical studies?

Since $m_{H}^{2}=\frac{2 \lambda}{\sigma_{a}^{2}} M_{W}^{2}$, it would appear that $m_{H}$ could be made arbitrarily small bv reducing $\lambda$. This is not the case since for very small $\lambda$ one must consider the effect of gauge interactions which induce Higgs self interactions at higher order. The Higgs self interactions are described by the effective potential ${ }^{\top}$

$$
V_{e f f}(\Phi)=-\mu^{2} \Phi^{+} \Phi+\lambda\left(\Phi^{+} \Phi\right)^{2} .
$$

TAlthough the effective potential is not gauge invariant, its minimum is. Consequenily the subsequent discussion which relr,tes only to the minimum is physically meaningful. 


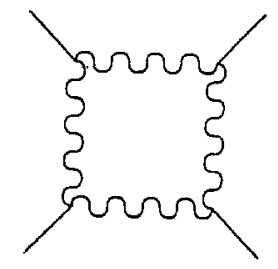

Figure 15: Feynman diagram showing a contribution to the effective potential for the Higgs field due to interactions of the Higgs with $W$ bosons.

Radiative corrections from Feynman diagrams of the type indicated in Fig. 15 modify this potential. At one loop $V_{\text {eff }}(\Phi)$ becomes ${ }^{32}$

$$
V(\Phi)=-\mu^{2}\left(\Phi^{+} \Phi\right)+\lambda\left(\Phi^{+} \Phi\right)^{2}+c\left(\Phi^{+} \Phi\right)^{2} \log \left(\left(\Phi^{+} \Phi\right) / M^{2}\right)
$$

where

$$
c=\frac{1}{16 \pi^{2} v^{4}}\left(3\left(2 M_{W}^{2}+M_{Z}^{2}\right)+m_{H}^{4}-4 \sum_{j} m_{f}^{4}\right)
$$

and $M$ is a renormalization scale. The Higgs mass $m_{H}$ is given by

$$
m_{H}^{2}=\left.\frac{\partial^{2} V}{\partial \Phi^{2}}\right|_{\phi=\{(\phi)}
$$

In general $V_{\text {eff }}$ will have more than one minimum. If we require that the minimum with $\langle\Phi\rangle \neq 0$ is lower than that with $\langle\Phi\rangle=0$ (the phase in which the $W$ boson remains massless), so that this phase will be the true ground state, then a bound on $\lambda$, and hence $m_{H}$, can be obtained since all the other quantities in Eq. (2.2) are known. We have ${ }^{33}$

$$
m_{B} \geq 7 \mathrm{GeV} \text {. }
$$

A more detailed study which requires that the universe not be trapped a: $\langle\Phi\rangle=0$ for too long ${ }^{34}$ gives $m_{H} \geq 10 \mathrm{GeV}$. This bound is extremely model dependent. A similar bound will exist in models with different Higgs sectors. ${ }^{35}$ In models with an arbitrary number of Higgs doublets there must be at least one physical Higgs boson with a mass greater than this bound.

As $\lambda$ is increased $m_{H}$ increases. Eventually $\lambda$ will become too large for the perturbative formula tor the Higgs mass to be valid. We can estimate this value naively by requiring that $\lambda^{2} / 4 \pi$ be less than one. This implies $m_{H} \$ 600 \mathrm{GeV}$. In order to be more precise it is necessary to consider the effects of the constraints imposed by partial wave unitarity. ${ }^{38}$

Consider the $S$ matrix for a two-particle scattering process $a+b \rightarrow c+d$. Unitarity requires that

$$
S^{+} S=1 .
$$

Writing $S=1+i T$, we have

$$
-I m T=T^{+} T
$$


The scattering matrix $T$ is given by

$$
T=(2 \pi)^{4} \delta^{4}\left(p_{a}+p_{b}-p_{c}-p_{d}\right) \frac{1}{(2 \pi)^{8}} \frac{1}{s}\left|M_{a b-c d}\right|^{2} .
$$

Here $p_{i}$ is the momentum of particle $i$ and $M_{i}$ the invariant matrix element obtained, for example, by calculating a set of Feynman diagrarrs. $M$ may be decomposed as follows:

$$
M(s, \cos \theta)=16 \pi \sum_{J=0}^{\infty}(2 J+1) A_{J}(s) P_{J}(\cos \theta) .
$$

$\theta$ is the center-of-mass scattering angle between particles $a$ and $c, P_{J}(\cos \theta)$ is a Legendre polynomial and $A_{J}(s)$ is some function. Equation 2.5 implies that

$$
\operatorname{Im} A_{0} \geq\left|A_{0}\right|^{2} .
$$

We can expand $A_{0}$ as a perturbation series in sorne coupling constant $g$

$$
A_{0}(s)=a_{1}(s) g^{2}+a_{2}(s) g^{4}+\ldots
$$

If the perturbation expression is reliable then

$$
g^{2}<a_{1} / a_{2} .
$$

The Born term $a_{1} g^{2}$ is real, hence Eq. 2.8 implies that

$$
-\operatorname{Im}\left(a_{2} g^{4}\right)>\left(a_{1} g^{2}\right) \text {. }
$$

But $|A|>|\operatorname{Im} A|$ for any $A$, so that the requirement that perturbation theory be reliable implies that

$$
\left|a_{1} g^{2}\right|<1 .
$$

Now this result can be applied to the process $H+H \rightarrow H+H$. If we assume that $m_{H}>M_{W}$, the relevant Feyiman diagrams are shown in Fig. 16 and give

$$
A_{0}(H H \rightarrow H H)=-\frac{G F m_{H}^{3}}{8 \pi \sqrt{2}}\left[3+\frac{9 m_{H}^{2}}{s-m_{H}^{2}}-\frac{2 m_{H}^{2}}{s-m_{H}^{2}} \log \left(s / m_{H}^{2}-3\right)\right] .
$$

Requiring $\left|A_{0}\right|<1$ (see Eq. (2.12)) in the limit $s \rightarrow \infty$ implies that

$$
m_{H}<1.7 \mathrm{TeV}
$$
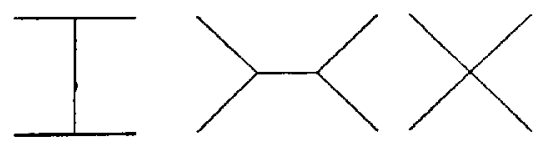

Figure 16: Feynman diagram for the process $H H \rightarrow H H$ which dominate in the limit $\pi_{H}>$ $m_{w}$. 
A stronger bound is obtained by considering the coupled channel problem: $H B \rightarrow$ $Z Z, H H \rightarrow W W, W W \rightarrow Z Z, H Z \rightarrow H Z, H W \rightarrow H W$. In this case one has ${ }^{30}$

$$
m_{H}<\sqrt{\frac{8 \pi \sqrt{2}}{3 G_{F}}}=.98 \mathrm{TeV} .
$$

This bound indicates that there must be a scalar particle of mass less than $1 \mathrm{TeV}$ or the Weinberg-Salam model will contain a strong, non-perturbative coupling. The presence of such a coupling implies that there must be non-perturbative structure in the $W W$ or $Z Z$ channel for $W W$ or $Z Z$ invariant masses of order $1 \mathrm{TeV}$. (Recall that the longitudinal components of the $W$ and $Z$ come from the Higgs fields.) General arguments which apply to strongly coupled systems can be used to predict these effects.

The basic argument that I have just outlined contains the essential features which justify the choices of energy and luminosity for the SSC. In order to probe the nature of the interactions responsible for the breakdown of the $S U(2) \times U(1)$ symmetry it is necessary to probe the $W W$ and $Z Z$ system with invariant masses of order $1 \mathrm{TeV}$.

A similar argument can be used to constrain the masses of heavy quarks or leptons which are proportional to Yukawa couplings. ${ }^{23,37}$ Consider the scattering of a quark or lepton $F_{i}$ of mass $m_{i}: F_{i} \bar{F}_{i} \rightarrow F_{j} \bar{F}_{j}$. There are contributions to the scattering amplitude from exchanges of $Z$ and Higgs bosons in the $s$ and $t$ channels. In the limit $s \rightarrow \infty$ with $m_{i}>>m_{H}, M_{Z}, M$ has the following form

$$
M=\sqrt{2} G_{F} m_{i} m_{j} \delta_{\lambda \lambda^{\prime}} \delta_{\lambda^{\prime} \lambda^{\prime}}\left[1-\lambda \lambda^{\prime}-2 \delta_{i j}\right] .
$$

Here $\lambda\left(\lambda^{\prime}\right)$ labels the helicity of the fermion $i(j)$ and $\bar{\lambda}$ labels the helicity of the anti-ferminns. The constraint of partial wave unitarity implies that

$$
m_{i}^{2}+m_{j}^{2}<\frac{\varepsilon \sqrt{2} \pi}{G_{F}} .
$$

If $m_{1}=m_{2}$ this implies that $m_{1} \$ 530 \mathrm{GeV}$. In the case of a heavy lepton of mass $m_{L}$ in a doublet with a massless neutrino: $m_{L} \leqslant 1.2 \mathrm{TeV}$. Quarks of masses larger than this cannot be discussed within the context of perturbation theory."

Let us now turn to the possible experimental signatures for Higgs bosons. The Higgs can decay to ferrsion anti-fermion $W W$ and $Z Z$ final states with the following partial widths:

$$
\begin{aligned}
\Gamma(H \rightarrow f \bar{f}) & =\frac{G_{F} m_{f}^{2} m_{H}}{4 \pi \sqrt{2}}(3)\left(1-4 m_{f}^{2} / m_{H}^{2}\right)^{3 / 2}, \\
\Gamma\left(H \rightarrow W^{+} W^{-}\right) & =\frac{G_{F} m_{H}^{3}}{32 \pi \sqrt{2}}\left(4-4 \epsilon+3 \epsilon^{2}\right)(1-\epsilon)^{1 / 2} \\
\Gamma(H \rightarrow Z Z) & =\frac{G_{F} m_{H}^{3}}{64 \pi \sqrt{2}}\left(4-4 \epsilon^{\prime}+3 \epsilon^{2}\right)\left(1-\epsilon^{\prime}\right)^{1 / 2} .
\end{aligned}
$$

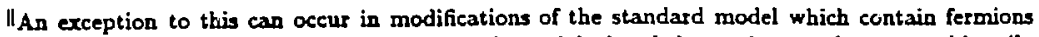
whose mass is not controlled by $(\Phi)$. For example, a right-handed neutrino can have an arbitrarily large Majorana mass. 


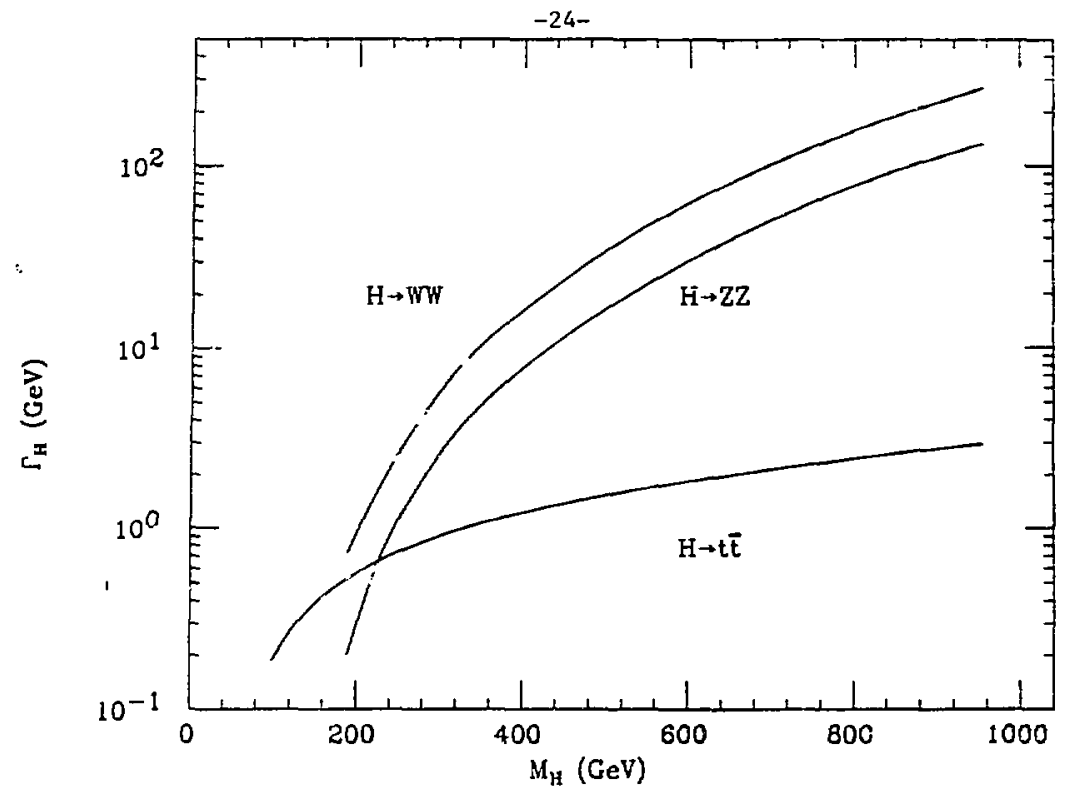

Figure 17: The partial widths $H \rightarrow t \vec{t}$ (solid lines), $W^{+} W^{-}$(dashed line) and $Z Z$ (dotied line) as a function of $\mathrm{m}_{\mathrm{H}}$.

with $\epsilon^{\prime}=4 M_{z}^{2} / m_{H}^{2}$ and $\epsilon=4 M_{W}^{2} / m_{H}^{2}$. The factor of 3 is included in the first expression only if $f$ is a quark. The implications of these formulae are easy to see. If $m_{H}<2 M_{W}$, the Higgs will decay dominantly into the heaviest fermion channel which is open. Once $\boldsymbol{m}_{H}$ is greater than $2 M_{W}$, the decay into two gauge bosons will dominate. This effect is shown in Fig. 17. Notice that the width grows rapidly as $m_{H}$ is increased. Eventually $\Gamma / m_{H} \sim 0(1)$ : this is another manifestation of the breakdown of perturbation theory at large values of $m_{H}$.

The Higgs can be produced in $e^{+} e^{-}$annihilation from the decay of a $Z$ through the graph shown in Fig. 18, with a rate shown in Fig. 19. The rate is given by ${ }^{38}$

$$
\begin{aligned}
\frac{1}{\Gamma\left(Z \rightarrow \mu^{+} \mu^{-}\right)} \frac{d \Gamma\left(Z \rightarrow H+\mu^{+} \mu^{-}\right)}{d x}=\frac{\alpha}{4 \sin ^{2} \theta_{W} \cos ^{2} \theta_{W}} . \\
\times \frac{\left(1-x+\frac{z^{2}}{12}+\frac{2 m_{x}^{2}}{3 M_{z}^{2}}\right)\left(x^{2}-\frac{4 m_{\mu}^{2}}{M_{Z}^{2}}\right)^{1 / 2}}{\left(x-\frac{m_{\alpha}^{2}}{M \mu_{2}^{2}}\right)^{2}}
\end{aligned}
$$

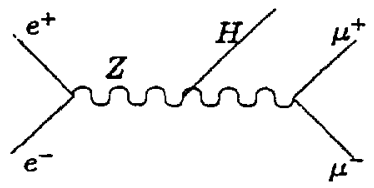

Figure 18: Feynman diagram for the process $e^{+} e^{-} \rightarrow Z \rightarrow H+$ $\boldsymbol{\mu}^{+} \boldsymbol{\mu}^{-}$. 


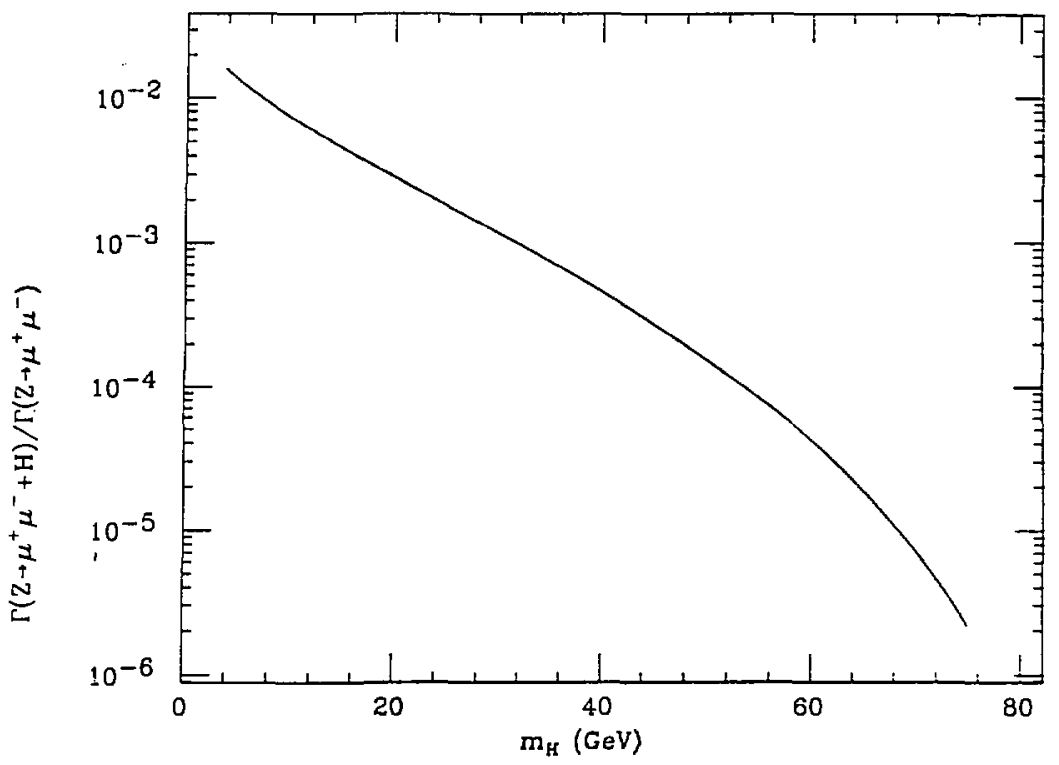

Figure 19: The ratio of widths $\Gamma\left(Z \rightarrow H_{\mu^{+} \mu^{-}}\right) / \Gamma\left(Z \rightarrow \mu^{+} \mu^{-}\right)$as a function of the Higgs mass.

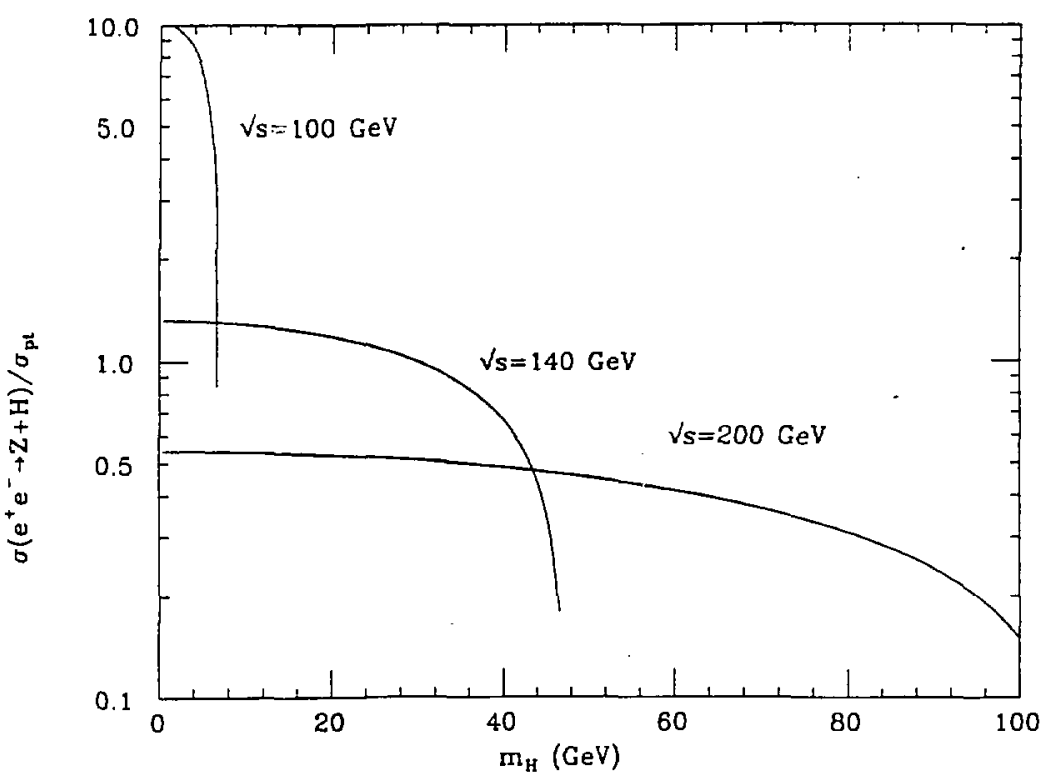

Figure 20: The cross-wction for the process $e^{+} e^{-} \rightarrow Z+H$ as a function of $m_{H}$ for various values of $\sqrt{s}$. 
Here $x=2 E_{H} / M_{Z}$ where $E_{H}$ is the energy of the Higgs boson. The rate is rather small, but the signature is very clean. It is not necessary to reconstruct the Higgs from its decay products; one searches for a peak in the mass recoiling against the lepton pair. Backgrounds arise from the production of a heavy quark pair if both of the quarks decay semileptonically. If we require that the leptons be isolated this background is not important. If one looks in the $e^{+} e^{-}$decay channel, then there is a background from the two photon process $e^{+} e^{-} \rightarrow e^{+} e^{-}+$hadrons which produces a serious problem for Higgs masses below about $8 \mathrm{GeV}$. A Higgs of mass less than $40 \mathrm{GeV}$ should be discovered at LEP/SLC using this process. If the Higgs mass exceeds $0.6 \mathrm{MZ}_{Z}$, then this rate is exceeded by that from $Z \rightarrow H+\gamma^{39}$ Again the signal is very clean, but the small rate makes it unlikely that this process will be observed.

The Higgs boson can also be produced at higher energies in $e^{+} e^{-}$annihilation via the process $e^{+} e^{-} \rightarrow Z+H, 40$ with the rate shown in Fig. 20. The production cross-section at center-of-mass energy $\sqrt{s}$ is given by

$$
\frac{d \sigma\left(e^{+} e^{-} \rightarrow Z+H\right)}{d(\cos \theta)}=\frac{\pi \alpha^{2}\left(1+8 \sin ^{4} \theta_{W}-4 \sin ^{2} \theta_{W}\right)}{16 \sin ^{4} \theta_{W} \cos ^{4} \theta_{W}\left(s-M_{Z}^{2}\right)^{2}} \frac{2 \kappa}{\sqrt{s}}\left(M_{Z}^{2}+\frac{\kappa^{2} \sin ^{2} \theta}{2}\right) .
$$

Here $\theta$ is the angle between the Higgs and the beam and $\kappa$ is the Higgs momentum. Again it is not necessary to reconstruct the final state arising from the Higgs decay. The cross section is not large, particularly if the $Z$ can only be detected via its decay to $\mu^{+} \mu^{-}$or $e^{+} e^{-}$. Nevertheless LEP should be able to probe Higgs masses up to $0.9\left(\sqrt{s}-M_{z}\right)$ using this mechanism.

Another potentially important process is the decay of the toponium bound state $(\theta)$ into $H+\gamma,{ }^{41}$ the rate for which is shown in Fig. 21. Since coupling of a Higgs to a quark is proportional to the quark mass, the rate will be largest if the top quark mass $\left(m_{\mathrm{t}}\right)$ is large. The rate is given by

$$
\frac{\Gamma\left(\theta \rightarrow H_{\gamma}\right)}{\Gamma\left(\theta \rightarrow \mu^{+}\right)}=\frac{G_{F} m_{L}^{2}}{\sqrt{2} \pi \alpha}\left(1-\frac{m_{H}^{2}}{m_{\theta}^{2}}\right)^{1 / 2} .
$$

This process has a rather large $Q C D$ correction. ${ }^{42}$ If this is included, the right-hand side of Eq. (2.20) is multiplied by

$$
\left(1-\frac{4 \alpha_{\vartheta}}{3 \pi} a\left(m_{H}^{2} / m_{\theta}^{2}\right)\right)
$$

Here, $a(x) \sim 10$ for $x \leq 0.8$, so that the correction reduces the naive rate. The branching ratio is reasonably large, but it is important to recall that the production rate for toponium in $e^{+} e^{-}$annihilation is not large. A $80 \mathrm{GeV}$ toponium state has a production cross section of order $0.1 \mathrm{nb}$.

The product of a Higgs in hadron-hadron collisions occurs via several mechanisms. Since the Higgs coupling to light quarks is very small, the production of Higgs bosons from the annihilation of light quark-antiquark pairs strongly suppressed. There are too few heavy quarks inside the proton for their annihilation to generate a reasonable rate. There are two important mechanisms. Firstly, the Higgs can be produced via gluon-gluon fusion according to the Feynman diagram shown in Fig. 22. ${ }^{43}$ This graph contains a vertex coupling the Higgs to a quark-antiquark pair, which is proportional to the quark mass. Consequently, the rate from this process depends sensitively 


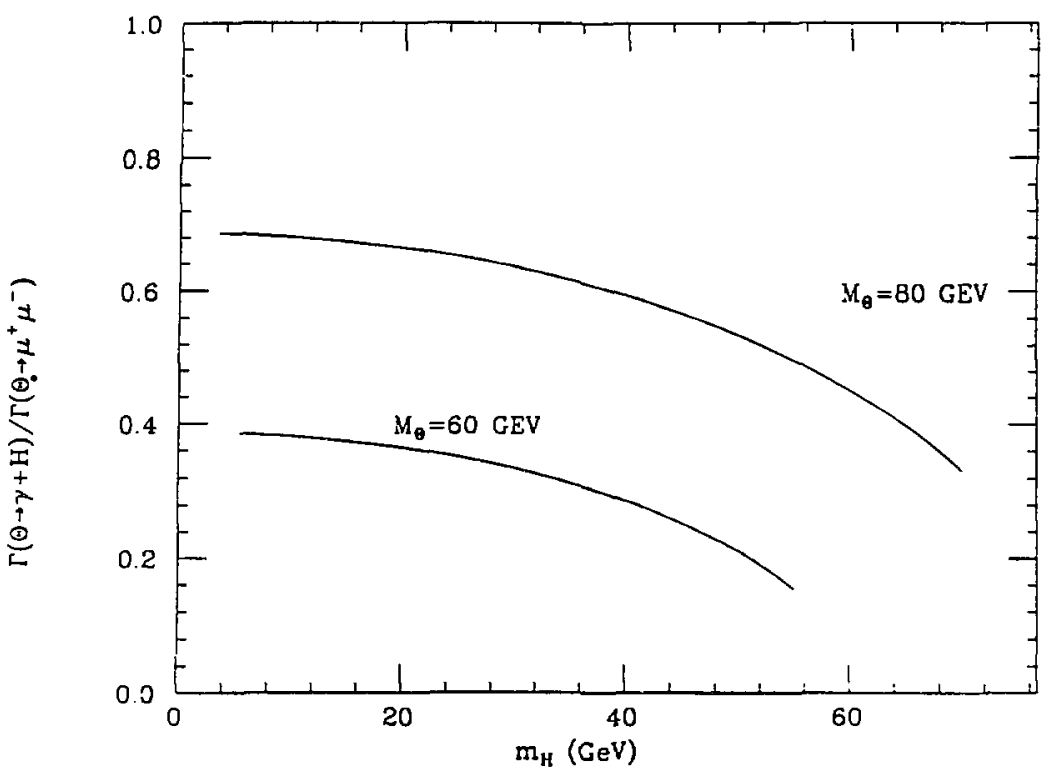

Figure 21: The ratio of decay widths $\Gamma(\theta \rightarrow H \gamma) / \Gamma\left(\theta \rightarrow \mu^{+} \mu^{-}\right)$for the decay of the $1^{--}$bound state $(\theta)$ of $t \bar{E}$. It has been assumed that $\theta$ has a mass of $80 \mathrm{GeV}$.

upon the mass of the top quark. The production rate at center-of-mass energy $\sqrt{s}$ is a proton-proton collision is given by

$$
\sigma(p p \rightarrow H+X)=\frac{G_{F} \pi}{32 \sqrt{2}}\left(\frac{\alpha_{s}}{\pi}\right)^{2} \eta^{2} \int_{M_{H}^{2} / \bullet}^{1} \frac{M_{H}^{2}}{s} g(x) g\left(m_{H}^{2} / s x\right) d x .
$$

$g(x)$ is the gluon distribution of a proton (see Sec. 3). Defining $\epsilon_{i}=4 m_{i}^{2} / m_{H}^{2}$ for a quark of mass $m_{i}, \eta$ is given by

$$
\eta=\sum_{i} \frac{\epsilon_{i}}{2}\left(1+\left(\epsilon_{i}-1\right) \phi\left(\epsilon_{i}\right)\right)
$$

with

$$
\phi(\epsilon)= \begin{cases}-\left[\sin ^{-1}(1 / \sqrt{\epsilon})\right]^{2} & \epsilon>1 \\ \frac{1}{4}\left(\log \left(-+/ \eta_{-}\right)+i \pi\right)^{2} & \epsilon<1\end{cases}
$$

where $\eta_{ \pm}=1 \pm \sqrt{1-\epsilon}$.

An alternative mechanism is shown in Fig. $23 .{ }^{44}$ At large values of $m_{H}$, the rate from this mechanism becomes large due to the large width for $H \rightarrow W W$. The exact formula for this rate is complicated; it simplifies drastically in the so-called effective $W$ approximation. This approximation assumes that the $W$ 's are emitted parallel to the incoming quarks and they are treated as if they are on mass-shell. It is similar to the effective photon approximation used to describe two-photon reactions in $e^{+} e^{-}$ annihilation where the electron beams are treated as sources of on-shell photons. In this approximation the cross-section for $q+q \rightarrow H+q q$ via intermediate $W$ 's is given $b y^{45}$ 


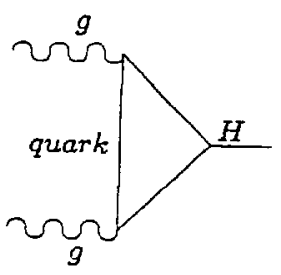

Figure 22: Feynman diagram showing the produsction of a Higgs boson via gluon-gluon fusion.

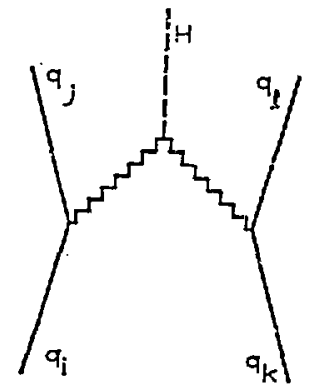

Figure 23: Feymman diagrain showing the process $q q \rightarrow H+q q$.

$$
\begin{aligned}
& \sigma\left(q_{i}+q_{j} \rightarrow H+q_{j}+q_{i}\right)=\frac{1}{16 M_{W}^{2}}\left(\frac{\alpha}{\sin ^{2} \theta_{W}}\right)^{3} \\
& \quad \times\left[\left(1+m_{H}^{2} / \hat{s}\right) \log \left(s / m_{H}^{2}\right)-2+2 m_{H}^{2} / \hat{s}\right] \theta\left(-e_{i} e_{j}\right)
\end{aligned}
$$

where $\sqrt{\hat{s}}$ is the center-of-mass energy of tha $q q$ system and $e_{i}$ is the charge of quark of type $i$. This may be converted into a hadronic cross-section via the parton model (see Sec. 3). In the case of intermediate $Z$ bosons the factor $\theta\left(-e_{i} e_{j}\right)$ is replaced by $\frac{1}{\cos \theta_{w}^{3}}\left(v_{i}^{2}+a_{i}^{2}\right)\left(v_{j}^{2}+a_{j}^{2}\right)$ where $v_{i}$ and $a_{i}$ were defined in Section 1 .

This mechanism will only be irnportant at the SSC; cross-sections evaluated at Tevatron and Spp $\bar{S}$ energies are dominated by the gluon fusion process. The rates for Higgs production are shown in Fig. 24. There are other mechanisms leading to final states with $H+Z, W+Z$ and $H+t \bar{t}{ }^{46}$ The rates for Higgs production via these mechanisms are smaller than those discussed above and will be useful only if the additional particles can be used as a tag in order to improve the signal-to-noise ratio. ${ }^{47}$

The signals for Higgs bosons at the SSC are discussed extensively elsewhere. 48,49 At the Tevatron the rates are reasonable only for Higgs nuasses less than $150 \mathrm{GeV}$ or so. In this mass region the Higgs will decay dominantly to $t \bar{t}$ if the $t$ quark is light enough. There is a large background from the $Q C D$ production of $t \bar{t}$ pairs (this will be discussed in the next section) which will make detection difficult even if the $t$ quark can be identified efficiently.

If the $t \bar{t}$ channel is not open, the Higgs will decay to $\tau^{+} \tau^{-}$with a branching ratio of $m_{r}^{2} / 3 \mathrm{~m}_{b}^{2} \sim 4.5 \%$. The only background source of $\tau$ pairs is Drell-Yan production $p \vec{p} \rightarrow \tau^{+} \tau^{-}+X$, via a virtual $Z$ or photon. Figure 25 shows the signal and background in the $\tau$ pair channel. I have assumed a resolution of $10 \mathrm{GeV}$ in the $\tau^{+} \tau^{-}$invariant mass. It can be seen that the signal to background ratio is rather poor. This figure assumes a top quark mass of $150 \mathrm{GeV}$. The tau final state can be identified from the one-prong tau decays $(\tau \rightarrow e \nu \nu, \mu \nu \nu, \pi \nu$, etc.). Energy is !ost into neutrinos so that the resolution in the $\tau^{+} \tau^{-}$invariant mass will be poor. The experiment is elearly very difficult. 


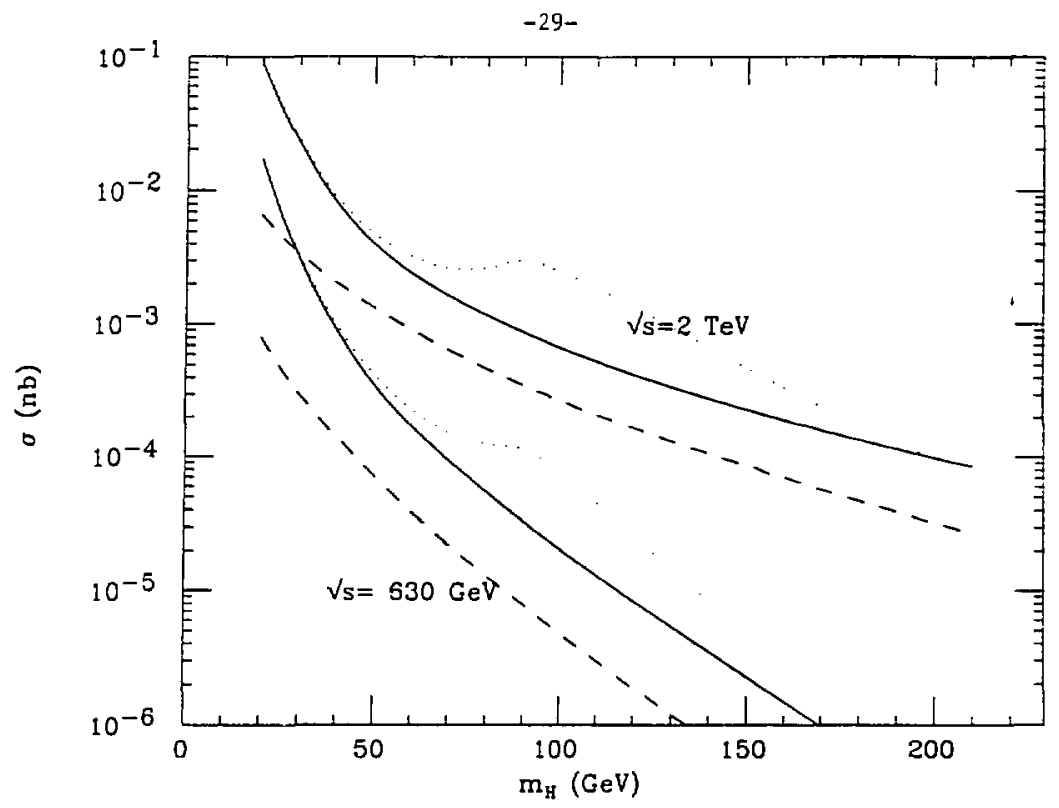

Figure 24: The cross-section $p \bar{p} \rightarrow H+X$ as a function of Higgs mass. The solid (dotted) lines correspond to the gluon fusion process of figure 22 with a top quark mass of 150 (40) GeV, and the dashed to the WW fusion process of figure 23.

There is one other possibility. The Higgs can decay to two photons (see Fig. 26) with the branching ratio ${ }^{50}$

$$
B R(H \rightarrow \gamma \gamma) \sim \frac{m_{\hat{H}}^{2}|A| \alpha^{2}}{6 \pi^{2} m_{b}^{2}} .
$$

I have assumed that $m_{\ell}>m_{H} / 2$. Here $A$ is a number arising from the $W$, quark and lepton loop diagram. Its value depends upon the masses of the particles involved but it is of order four for Higgs masses around $100 \mathrm{GeV}$. The background arises from the production of photon pairs via quark-antiquark collisions and is not too large. Unfortunately the branching ratio is so small that there are insufficiert events for this decay mode to be useful. It has been suggested ${ }^{\text {st }}$ as a possible mode at the SSC where the event rates are much larger.

What can we conclude about the prospects for finding the Higgs in the near future? If its mass is less than $40 \mathrm{GeV}$ or so, it should be found in the decay of the $Z$ either at the SLC or at LEP. Masses larger than this can be probed in the decay of toponium, if toponium exists in an accessible mass range. Notice that if $m_{t}>m_{b}+M_{W}$, the top quark will decay too quickly for narrow toponium bound states to exist. Higgs masses up to $100 \mathrm{GeV}$ can be probed in the early 1990's at LEP when the energy is increased to $100 \mathrm{GeV}$ per beam. Higgs bosons of mass greater than this will have to wait for the SSC. 


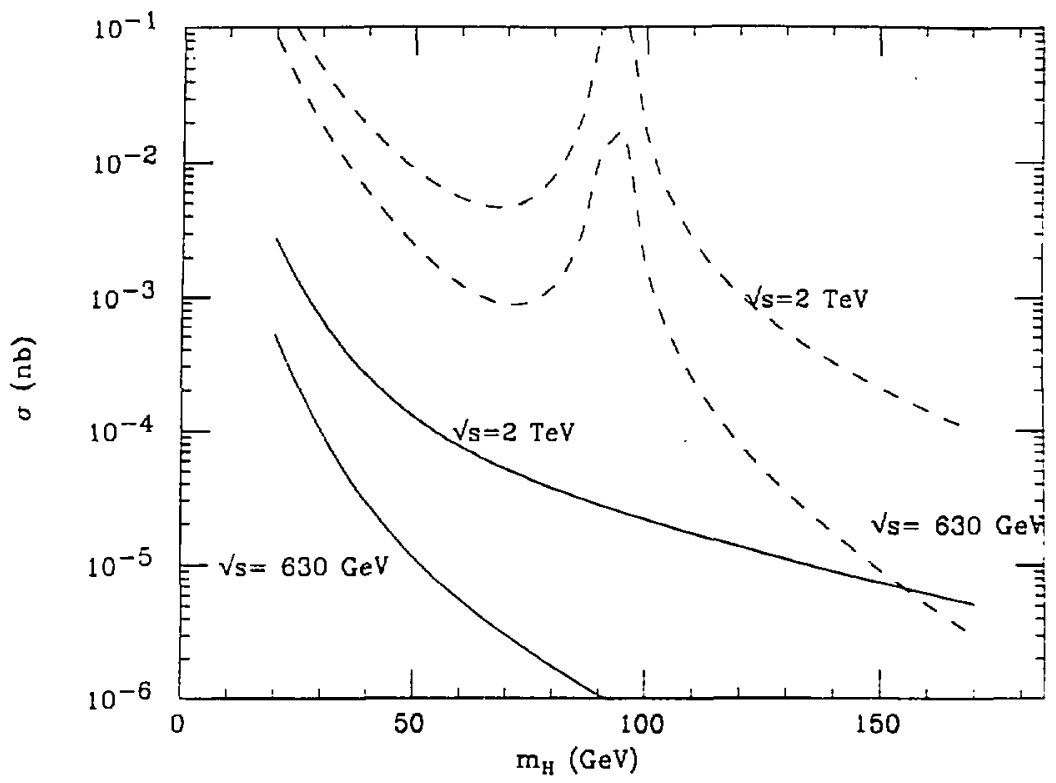

Figure 25: A comparison of the signal and background for the process $p \bar{p} \rightarrow H+X \rightarrow \tau^{+} \tau^{-}+X$. It is assumed that $m_{H}<2 m_{\mathrm{t}}$. The background is calculated from the Drell-Yan process (see Sect. 3) being $d \sigma / d M \Delta M$. The resolution in the invariant mass of the tau pair $(\Delta M)$ is taken to be $10 \mathrm{GeV}$.

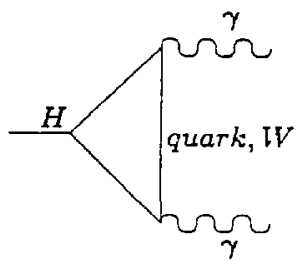

Figure 26: Feynman diagram for the process $H \rightarrow \gamma \gamma$. 


\section{QCD}

In this chapter, I shall provide an introduction to perturbative $Q C D$. I shall emphasize the uses of $Q C D$ in calculating rates at hadron-hadron colliders. Since $Q C D$ processes account for most of the background for new physics at such colliders, it is important to understand the uncertainties in these predicted rates. Given the limited time available I have had to be selective in the topics discussed.* I will begin with a discussion of the one parameter of $Q C D$, namely, its coupling constant. I shall then discuss the parton model in some detail. I will conclude with a discussion of the production of new quarks at hadron-hadron colliders. This discussion will serve as a framework for an analysis of some of the uncertainties in such calculations.

The $Q C D$ Lagrangian may be written as follows:

$$
-\frac{1}{4} F_{\mu \nu}^{i} F_{\mu}^{i}+\sum_{j} \vec{\psi}_{j}\left(i, \partial-m_{j}\right) \psi_{j}
$$

The sum on $j$ runs over quark flavors and,

$$
F_{\mu \nu}^{i}=\partial_{\mu} G_{\mu}^{i}-\partial_{\nu} G_{\mu}^{i}-i g f_{i j h} G_{\mu}^{i} G_{\mu}^{i}
$$

and

$$
D_{\mu}=\partial_{\mu}-i g t^{i} G_{\mu}^{i}
$$

Here $t^{i}$ are the $3 \times 3$ representation matrices and the structure constant $f_{i j k}$ are given by $\left[t_{i}, t_{j}\right]=i f_{i j k} t_{k}$.

Apart from the quark masses, which have their origin in the Weinberg-Salam model, the theory has only one fundamental parameter, the coupling constant $g$. As in the case of the electroweal theory, beyond tree level it is necessary to define a renormalized coupling constant $g(\mu)$. In the case of $Q E D$ this could be done in terms of the static potential between two electrons. The analogous definition in $Q C D$ would be in terms of the inter-quark potential. In the case of light quarks such a definition is :mpossible in the context of a perturbative theory since $Q C D$ is strongly coupled at such low momentum scales. A definition in terms of the potential between two heavy quarks is possible but not particularly convenient. I shall therefore use the modified minimal subtraction scheme discussed in Sect. I (see Eq. (1.11)).

Let us calculate a physical process $P\left(Q^{2}\right)$, which depends on some energy scale $Q$; $P$ could, for example, represent a cross-section. If we neglect quark masses, calculate in $n$ dimensions then

$$
P\left(Q^{2}\right) \sim\left[\frac{2 A}{4-n}-A \gamma_{E}+A \log 4 \pi-F\left(\mu, Q^{2}, g\right)\right]
$$

Recall that the scale $\mu$ is introduced so that the coupling constant $g$ remains dimensionless in $n$ dimensions, viz.,

$$
g \rightarrow g \mu^{(4-n) / 2}
$$

It is convenient to choose the quantity $P$ to be dimensionless; this can always be done by multiplying it by an appropriate power of $Q$. Then $P$ must have the form, after subtraction of the $1 /(n-4), \gamma_{E}$ and $\log 4 \pi$ terms

$$
P\left(Q^{2}\right)=F\left(Q^{2} / \mu^{2}, \alpha\right)
$$

I have replaced $g$ by $\alpha: \alpha \equiv g^{2} / 4 \pi$. Now, the scale $\mu$ is arbitrary so that a physical quantity cannot depend upon its value 


$$
\frac{d P}{d \mu}=0
$$

which implies

$$
\left(\mu^{2} \frac{\partial F}{\partial \mu^{2}}+\beta(\alpha) \frac{\partial F}{\partial \alpha}\right)=0
$$

Here $\beta(\alpha)$ is defined by

$$
\beta(\alpha) \equiv \mu^{2} \frac{\partial \alpha}{\partial \mu^{2}}
$$

(Recall that the bare coupling $\alpha$ depends on $\mu$ (see Eq. (1.11).) We can introduce a momentum-aependent coupling $\alpha(t)$ via

$$
t \equiv \int_{\alpha}^{\alpha(t)} \frac{d p}{\beta(p)}
$$

where $t=\log \left(Q^{2} / \mu^{2}\right)$ Then Eq. (3.6) has the solution

$$
F(t, \alpha)=F(1, \alpha(t))
$$

Hence the only dependence on the scale $Q$ or $t$ is carried by $\alpha(t)$. We can expand $\beta$ as a power series in $\alpha$.

$$
\beta=-b \frac{\alpha}{4 \pi}-b^{\prime}\left(\frac{\alpha}{4 \pi}\right)^{2}+\cdots
$$

Hence $\alpha\left(\mu^{2}\right)$ has the following form:

$$
\alpha\left(\mu^{2}\right)=\frac{4 \pi}{b \log \left(\mu^{2} / \Lambda^{2}\right)}+\cdots
$$

Here $b=11-2 n_{f} / 3$ where $n_{f}$ is the number of quark flavors with mass less than $\mu$. We can regard the fundamental parameter of $Q C D$ either as $\alpha\left(Q_{0}^{2}\right)$ or as the scale $\Lambda$. Notice that as $\mu$ becomes small, $\alpha$ becomes large. Therefore, perturbation theory cannot be used to discuss processes which involve momentum flows as small as a few times $\Lambda$.

The value of $\Lambda$ or $\alpha\left(Q_{0}^{2}\right)$ which has been obtained is dependent upon the renormalization scheme used. For example, I could have used minimal subtraction, in which case the $\log (4 \pi)$ and $\gamma_{E}$ would not have been removed. The expression for $P$ written in terms of the new coupling constant $\bar{\alpha}$ can be used to express $\bar{\alpha}$ in terms of $\alpha$, since the value of a physical quantity cannot depend on the scheme

$$
\begin{aligned}
P(\bar{\alpha})=P(\alpha) \Rightarrow \bar{\alpha} & =f(\alpha) \\
& =\alpha+c \alpha^{2}+\ldots
\end{aligned}
$$

which corresponds to a new value of $\Lambda$

$$
\bar{\Lambda}=\Lambda e^{c / 2 b}
$$

A physical quantity is, of course, independent of the renorrnalization scheme. However, if the series is terminated at some finite order in the coupling constant, the values of $P\left(P_{N}\right)$ calculated to this order wiil differ

$$
P_{N}(\ddot{\alpha}) \neq P_{N}(\alpha)=P_{N}(\bar{\alpha})+0\left(\alpha^{N+1}\right)
$$




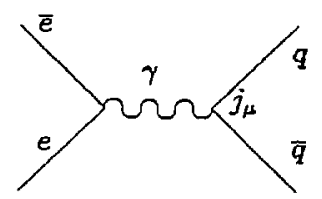

Figure 27: Feynman graph for $e^{+} e^{-} \rightarrow$ hadrons.

Since the coupling constant of $Q C D$ is not very small and most processes are not known to a very high order, these differences can be significant.

As a specific example of $Q C D$ process, consider the total cross-section for $e^{+} e^{-} \rightarrow$ hadrons at center-of-mass energy $\sqrt{s}$. In the one photon approximation (see Fig. 27) this is given by

$$
\sigma_{\text {had }}=\frac{8 \pi \alpha_{e m}^{2}}{3 s^{2}} \sum_{n}(2 \pi)^{4} \delta\left(q-q_{n}\right)\left\langle 0\left|j_{\mu}\right| n\right\rangle\left\langle n\left|j_{\mu}\right| 0\right\rangle
$$

where $j_{\mu}$ is the electromagnetic current of the quarks

$$
j_{\mu}=\sum_{i} e_{i} \bar{\psi}_{i} \gamma_{\mu} \psi_{i}
$$

If we introduce the photon self-energy function $\Pi^{\mu \nu}$

$$
\Pi_{\mu \nu}(q)=i \int d^{4} x e^{i q x}\left\langle\left|T\left(j_{\mu}(x) j_{\mu}(0)\right)\right| 0\right\rangle
$$

Defining $\Pi_{\mu, \nu}(q)=\left(g_{\mu, \nu} q^{2}-q_{\mu} q_{\nu}\right)=\Pi\left(Q^{2}\right)$ then

$$
\sigma_{\text {had }}=\frac{16 \pi^{2} \alpha_{\mathrm{em}}^{2}}{s} \operatorname{Im} \Pi(s)
$$

A dimensionless quantity is $R(s)$ defined by

$$
R(s)=\frac{\sigma_{\text {had }}}{\sigma\left(e^{-} e^{+} \rightarrow \mu^{+} \mu^{-}\right)} .
$$

Apart from the scale $\sqrt{s}, R$ could a'so depend upon $m_{i}^{2} / s$, where $m_{i}$ is 'he mass of a quark of type $i$. The dependence of $R$ upon $m_{i}$ can be seen by analyzing the Feynman diagram of Fig. 27. The graph is not singular as $m_{i}$ tends to zero,

$$
R_{m_{i} \rightarrow 0}=\text { const }+\frac{m_{i}^{2}}{s} \log m_{i}^{2}
$$

We can therefore neglect all light quark masses, $R$ is then a function only of $s / \mu^{2}$, and the previous argument implies that $R=R(\alpha(s))$. If we calculate $R$ using perturbation theory we get

$$
R=\sum e_{i}^{2}\left(1+\frac{\alpha}{\pi}+B\left(\frac{\alpha}{\pi}\right)^{2} \ldots\right)
$$

where $B$ is a scheme-dependent constant which is small ${ }^{54}$ in the $\overline{M S}$ scheme. 
Why are the non-perturbative effects irrelevant? After all, the final state consists of hadrons rather than free quarks which are used in the perturbative calculation. This can be understood by considering the time evolution of the final state. At very early times (equivalently large momenta) $Q C D$ is a weally coupled theory so perturbation theory should be reliable. At large $q$ the exponential in Eq. (3.17) is rapidly oscillating so only small values of $x_{\mu}$ contribute to $\Pi(q)$. Hence we are dominated by short distances (times). The hadronization of the final state takes place at later times (or order $1 / \Lambda$ ) and although it can affect detailed properties of the final state, it is incapable of modifying the total cross-section.

In order to discuss processes which involve hadrons in the initial state, we must discuss the parton model. ${ }^{52}$ Consider the case of electron-proton scattering, where the cross-section can be written as

$$
\frac{d \sigma}{d x d y}=\frac{4 \pi \alpha_{e m}^{2} s}{Q^{4}}\left[\frac{1+(1-y)^{2}}{2} 2 x F_{1}\left(x, Q^{2}\right)+(1-y)\left(F_{2}\left(x, Q^{2}\right)-2 x F_{1}\left(x, Q^{2}\right)\right)\right]
$$

The variables are defined as follows (see Fig. 28): $q$ is the moment um of the exchanged photon and $P$ is not momentum of the target proton and $k$ is that of the incoming electron

$$
\begin{aligned}
Q^{2} & =-q^{2} \\
\nu & =\frac{q \cdot P}{M_{p}} \\
x & =\frac{Q^{2}}{2 m_{p} \nu} \\
y & =\frac{q \cdot p}{k \cdot p} \\
s & =2 p \cdot k+m_{p}^{2}
\end{aligned}
$$

where $m_{\mathrm{p}}$ is the proton mass. I have neglected parity violating effects which arise from the exchange of a $Z$ boson instead of a photon.

In the naive parton model the proton is viewed as being made up of a set of noninteracting partons. The structure functions $F_{1}$ and $F_{2}$ are related to the probability distribution $q_{i}(x)$ which represents the probability of finding a parton of type $i$ (quark or gluon) inside the proton with fraction $x$ of the proton's momentum, and the scattering cross-section for such a virtual photon from a parton.

$$
F_{1}=\frac{F_{2}}{2 x}=\sum_{i} \int_{x}^{1} \frac{d y}{y} q_{i}(y)\left[e_{i}^{2} \delta(x / y-1)\right]
$$

where $e_{i}$ is the charge of parton of type $i$. The $\delta$-function appears from the crosssection for $q+\gamma \rightarrow q$. Let us consider $Q C D$ corrections to this scattering. At next order in $\alpha_{,}$, there are contributions from gluon emission which lead to the final state $q+g$ and also from virtual gluons (see Fig. 29). To order $\alpha_{*}(3.24)$ is replaced by

$$
F_{1}=\sum_{i} \frac{d y}{y} q_{i}(y)\left[e_{i}^{2} \delta\left(\frac{x}{y}-1\right)+\sigma_{i}\left(\frac{x}{y}, Q^{2}\right)\right]
$$

with 

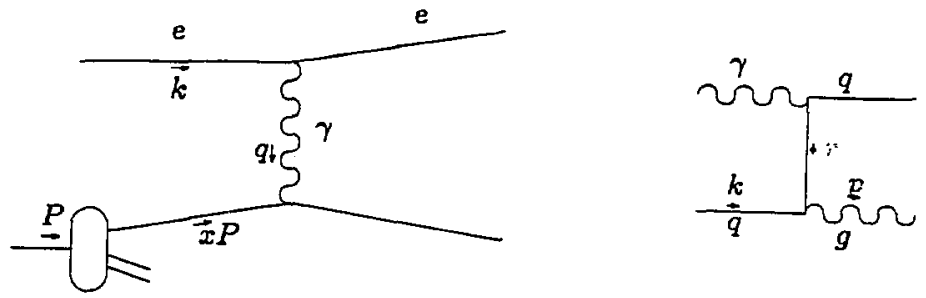

Figure 28: Diagram illustrating the vari- Figure 29: Diagram contributing to the ables in deep inelastic scattering (see Eq. (3.22)): elecprocess $q+\gamma \rightarrow X$ at order tron + proton $\rightarrow$ electron $\alpha_{\text {. }}$ + anything.

$$
\sigma_{i}\left(z, Q^{2}\right)=\frac{\alpha_{s}}{2 \pi} e_{i}^{2}\left[t P_{q Q}(z)+f(z)+0\left(\frac{1}{Q^{2}}\right)\right]
$$

with

$$
P_{9 q}(z)=\frac{4}{3} \frac{\left(1+z^{2}\right)}{1-z}
$$

for $z \neq 1$. Here $t=\log \left(Q^{2} / \mu^{2}\right)$ and the scale $\mu$ has appeared from dimensional regularization (I have dropped terms $1 /(n-4)$ ). The $\mu$ dependence arises because $\sigma_{i}$ is not finite in four dimensions. In the cases discussed previously, the divergences arise from large momentum flows inside loop diagrams (ultra-violet divergences). In this case these divergences cancel. Individual Feynman diagrams can also have divergences when momentum flows become very small or partieles are collinear. The former (soft) divergences cancel between the real and the virtual diagrams but the collinear ones do not. In order to see the origin of the problem consider the graph of Fig. 29 and work in a frame where $k_{\mu}=(k, k, 0,0)$.

If the transverse momentum of the gluon $(p)$ relative to $k$ is small then we can take $p=\left(\eta k+k_{\perp}^{2} / 2 \eta k, \eta k, k_{\perp} 0\right)$. (Terms of order $k_{\perp}^{2}$ are neglected.) The internal quark line now has invariant mass squared $r^{2}=(k-p)^{2}=k_{\perp}^{2} / \eta$, so that the squared amplitude from the graph will contain $1 / k_{1}^{4}$. Now at very small $k_{\perp}$ helicity conservation forbids the emission of a real gluon from a quark line, so that one factor of $k_{\perp}^{2}$ appears in the numerator. We now have for the total cross-section $q+\gamma \rightarrow q+$ anything, a contribution

$$
\sigma \sim \frac{\alpha,}{2 \pi} \int \frac{d k_{1}^{2}}{k_{\perp}^{2}}
$$

which gives rise to a logarithmic singularity. Notice that for a massive quark the singularity becomes $\log \left(Q^{2} / m_{q}^{2}\right)$.

We have obtained a result which depends on $\mu$ (or contains the large log $\left(Q^{2} / \mathrm{m}_{i}^{2}\right.$ ) if quark masses are retained). This is not physically meaningful. But Eq. (3.25) contains the unknown quantity $q_{i}(y)$. We can define $e^{55}$

$$
q_{i}(x, t)=q_{i}(x)+\frac{\alpha, t}{2 \pi} \int_{x}^{1} \frac{d y}{y} q(y) P_{q q}\left(\frac{x}{y}\right)
$$

Hence

$$
F_{i}=\sum_{i} \int_{x}^{l} e_{i}^{2} \frac{d y}{y} q_{i}(y)\left[\delta\left(\frac{x}{y}-1\right)+\frac{\alpha_{0}}{2 \pi} f\left(\frac{x}{y}\right)\right]+0\left(\alpha^{2}\right)
$$


The $t$ dependence can be eliminated at the cost of introducing a $t$-dependent structure function

$$
\frac{d}{d t} q(x, t)=\frac{\alpha_{s}}{2 \pi} \int_{x}^{1} \frac{d y}{y} q(y, t) P_{q q}\left(\frac{x}{y}\right)+0\left(\alpha_{s}^{2}\right)
$$

I have so far considered an oversimplification of the true problem. To order $\alpha_{\text {, }}$ there is an additional partonic process, namely gluon $+\gamma \rightarrow q+\bar{q}$ (see Fig. 30). This process also contains a $\log \left(Q^{2} / \mu\right)$ arising from the propagation of the internal quark close to its mass shell. This singularity results in the replacement of Eq. (3.25) and (3.26) by

$$
\begin{aligned}
F_{1}(x, t) & =\int_{x}^{1} \frac{d y}{y}\left[\sum _ { i } e _ { i } ^ { 2 } q _ { i } ( y ) \left[\delta\left(\frac{x}{y}\right)+\frac{\alpha_{a}}{2 \pi}\left[t P_{q q}\left(\frac{x}{y}\right)+f_{q}\left(\frac{x}{y}\right)\right]\right.\right. \\
& \left.+\left(\sum_{i} e_{i}^{2}\right) g(y) \frac{\alpha_{d}}{2 \pi}\left[t P_{q g}\left(\frac{x}{y}\right)+f_{\nabla}\left(\frac{x}{y}\right)\right]\right]
\end{aligned}
$$

with $P_{q q}(x)=1 / 2\left(x^{2}+(1-x)^{2}\right)$. The $t$ dependence can be absorbed by defining

$$
q_{i}(x, t)=q_{i}(x)+\frac{\alpha_{4}}{2 \pi} t \int_{x}^{1}\left(q_{i}(y) P_{q q}\left(\frac{x}{y}\right)+g(y) P_{Q 8}\left(\frac{x}{y}\right)\right) \frac{d y}{y}
$$

so that the quark and gluon distributions $\left(q_{i}(x)\right.$ and $\left.g(x)\right)$ are now coupled.

Given data from which $q_{i}\left(x, t_{0}\right)$ and $g\left(x, t_{0}\right)$ can be obtained as functions of $x$ for a fixed $t_{0}$, the equations for the evolution of $g(x, t)$ and $g(x, t)$ with $t$ (cf. 3.30) can be solved to obtain them for all $t$.

A vital property of $Q C D$ is that the distribution functions defined by (3.32) are universal. In order to illustrate this, consider the Drell-Yan process in proton-proton collisions. In the naive parton model, the cross-section for the production of a $\mu^{+} \mu^{-}$ pair of invariant mass. $I /$ in a proton-proton collision ‥: :ch total center-of-mass energy $\sqrt{s}$ is given by

$$
\frac{d \sigma}{d M^{2}}=\frac{4 \pi \alpha_{e m}^{2}}{9 M^{2} s} \int d x_{1} d x_{2 i}\left(\sum_{i} q_{1}\left(x_{1}\right) \bar{y}_{1}\left(x_{3}\right) e_{1}^{2} \varepsilon\left(x_{1} x_{2}-. M^{2} / s\right)+(1 \Leftrightarrow 2)\right)
$$

Here $\bar{q}$ is an antiquark distribution. Th. ?undamental process is quark-antiquark annihilation into $\mu^{+} \mu^{-}$. Consider the corrections to this at order $\alpha_{s}$. As in the case
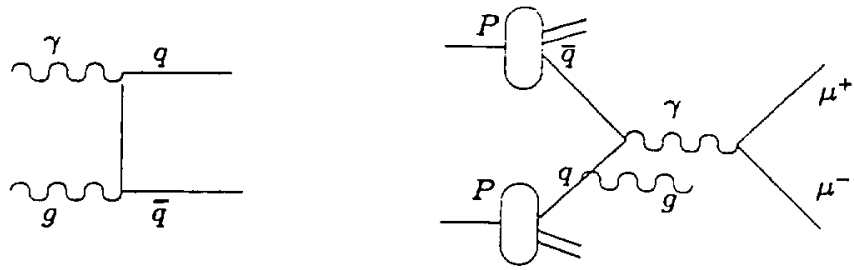

Figure 30: Diagram showing $g+\gamma \rightarrow$ $q+\bar{q}$.
Figure 31: Feynman graph illustrating an order $\alpha_{\theta}$ contribution to the Dress-Yan process (see Eq. (3.34)). 
of ep scattering these can involve either virtual or real gluons (see Fig. 31). These corrections modify Eq. (3.33), viz.,

$$
\begin{aligned}
\frac{d \sigma}{d M^{2}}= & \frac{4 \pi \alpha_{m}^{2}}{9 M^{2} s} \int \frac{d x_{1}}{x_{1}} \frac{d x_{2}}{x_{2}}\left[\left[e_{i}^{2} q_{i}\left(x_{1}\right) \vec{q}_{i}\left(x_{2}\right)+(1 \Leftrightarrow 2)\right]\right. \\
& {\left[\delta(1-z)+\theta(1-z) \frac{\alpha_{i}}{2 \pi}\left[2 P_{q q}(z) t+f^{\prime}(z)\right]\right] } \\
+ & {\left[\sum_{i} e_{i}^{2}\left(q_{i}\left(x_{1}\right)+\bar{q}_{i}\left(x_{1}\right)\right) G\left(x_{2}\right)+(1 \Leftrightarrow 2)\right] } \\
& {\left[\theta(1-z) \frac{\alpha_{s}}{2 \pi}\left[P_{q q}(z)+f^{\prime \prime}(z)\right]\right.}
\end{aligned}
$$

where $z=M^{2} /\left(s x_{1} x_{3}\right){ }^{58}$ The last part of the expression arises from the process $g+q \rightarrow \mu^{+} \mu^{-}+q$.

If we replace $q(x)$ by $q(x, t)$ defined by Eq. (3.32) then the resulting expression will have no $t$ 's appearing explicitly, viz.,

$$
\frac{d \sigma}{d M^{2}}=\frac{4 \pi \alpha_{e m}^{2}}{9 M^{2} s} \int d x_{3} d x_{2}\left[e_{i}^{2} g_{i}\left(x_{1}, t\right) \bar{q}_{i}\left(x_{2}, t\right) \delta\left(x_{1} x_{2}-M^{2} / s\right)+(1 \Leftrightarrow 2)+\mathcal{O}\left(\alpha_{s}\left(Q^{2}\right)\right)\right]
$$

where the order $\alpha_{\text {, }}\left(Q^{2}\right)$ terms contain no powers of $t$. This absorption of the singular terms into $q(x, t)$ is known as factorization; it is a universal property which guarantees that hard processes can be relilably calculated in perturbative $Q C D$.

I would now like to discuss some of the errors and uncertainties present in predictions of the rate for $Q C D$ processes in hadron-hadron collisions. I will discuss the production of new heavy quarks. A similar discussion applies to most other processes. The relevant $Q C D$ processes for the production of a $Q \bar{Q}$ pair are $g g \rightarrow Q \bar{Q}$ or $\bar{q} q \rightarrow Q \bar{Q}$. The cross-sections are given by ${ }^{57}$

$$
\begin{aligned}
\frac{d \sigma}{d t}(g g \rightarrow Q \bar{Q})=\frac{\pi \alpha_{s}^{2}\left(Q^{2}\right)}{8 s^{2}} & \left\{\frac{6}{s^{2}}\left(t-M_{Q}^{2}\right)\left(u-M_{Q}^{2}\right)+\left[\left(\frac{4}{3} \frac{\left(t-M_{Q}^{2}\right)\left(u-M_{Q}^{2}\right)-2 M_{Q}^{2}\left(t+M_{Q}^{2}\right)}{\left(t-M_{Q}^{2}\right)^{2}}\right.\right.\right. \\
& \left.\left.+\frac{3\left(t-M_{Q}^{2}\right)\left(u-M_{Q}^{2}\right)+M_{Q}^{2}(u-t)}{s\left(t-M_{Q}^{2}\right)}\right)+[t-u]\right] \\
& \left.-\frac{M_{Q}^{2}\left(s-4 M_{Q}^{2}\right)}{3\left(t-M_{Q}^{2}\right)\left(u-M_{Q}^{2}\right)}\right\}
\end{aligned}
$$

and

$$
\frac{d \sigma}{d t}(q \bar{q} \rightarrow q \bar{q})=\frac{\pi \alpha_{s}^{2}\left(Q^{2}\right)}{9 s^{2}}\left[\frac{\left(t-M_{Q}^{2}\right)^{2}+\left(u-M_{Q}^{2}\right)^{2}+2 M_{Q}^{2}}{s^{2}}\right]
$$

Here $s, t$ and $u$ are the usual Mandelstam variables. The rate for $p p \rightarrow Q \bar{Q}+$ anything when the quark emerges with transverse momentum $p_{\perp}$ at angle $\theta$ to the beam in the center-of-mass frame of the $p p$ system is

$$
E \frac{d \sigma}{d^{3} p}=\frac{1}{\pi} \sum_{i j} \int_{x_{\min }}^{1} \frac{d x_{a}}{x_{a}-x_{\perp}\left[\frac{x+\cos \theta}{2 \sin \theta}\right]} x_{a} x_{b} f_{i}\left(x_{a}, Q^{2}\right) f_{j}\left(x_{b}, Q^{2}\right) \frac{d \sigma}{d \hat{t}}(\hat{s}, \hat{t}, \hat{u})
$$

where 


$$
\begin{aligned}
& \hat{s}=x_{a} x_{b} s \\
& \hat{t}=M_{Q}^{2}-x_{a} x_{\perp} s\left[\frac{\chi-\cos \theta}{2 \sin \theta}\right] \\
& \hat{u}=M_{Q}^{2}-x_{b} x_{\perp} s\left[\frac{\chi+\cos \theta}{2 \sin \theta}\right]
\end{aligned}
$$

and

$$
\begin{aligned}
x_{b} & =\frac{x_{2} x_{\perp} s(\chi-\cos \theta)}{s \sin \theta\left(2 x_{a}-x_{\perp}\left[\frac{x+\cos \theta}{2 \sin \theta}\right]\right)} \\
x_{\min } & =\frac{x_{\perp}(\chi+\cos \theta)}{s \sin \theta\left(2-x_{\perp}\left[\frac{x-\cos \theta}{2 \sin \theta}\right]\right)} \\
x & =\left(1+\frac{4 M_{Q}^{2} \sin ^{2} \theta}{x_{\perp}^{2} s}\right)^{1 / 2} \\
x_{\perp} & =2 p_{\perp} / \sqrt{s}
\end{aligned}
$$

In order to use this formula we must; fix the structure functions; determine the scale $Q^{2}$ appearing in the structure functions; fix the scale appearing in $\alpha_{s} ;$ and define the quark mass. Let us discuss these problems in turn.

The structure functions are extracted at low $Q^{2}$ from the scattering of electrons or neutrinos off hadronic targets. These processes can only measure the quark structure functions directily since the gluons have no electroweal interactions. The gluon distributions must be inferred fron the $Q^{2}$ dependence of the quark distribution (see Eq. (3.32)). This implies that there is a correlation between $g(x)$ and the value of $\alpha$, which controls the $Q^{2}$ dependence.

For processes at hadron colliders, the required values of $Q^{2}$ are larger than those at which the distribution functions are measured; most electron and neutrino scattering experiments have most of their statistics for $Q^{2}<10 \mathrm{GeV}^{2}$. The distribution functions are then evolved up to larger $Q^{2}$ using $Q C D$. In this evolution some of the uncertainties tend to wash out. This is illustrated in Fig. 32 which compares two sets of structure functions at different $Q^{2} .58 .59$

In the case of $Q \vec{Q}$ production, the rate from gluon-gluon collisions dominates. There are two reasons for this: the gluon distribution is larger than that for quarks at small $x$ (see Fig. 32) and the process $g g \rightarrow Q \bar{Q}$ has a larger rate than $q \bar{q} \rightarrow Q \bar{Q}$ due to the higher color charge of the gluon. If, however, in $p \bar{p}$ collisions, we produce quarks of very large mass, the appropriate values of $x_{a}$ and $x_{b}$ (see Eq. (3.38)) can become large and we are forced into a region where $g(x)<q(x)$ so that the quart: antiquark annihilations can dominate. Notice that at these large values of $x$ the distribution functions and hence the cross-sections are small.

Other data from hadron-hadron collisions can be used to check that the gluon distributions are reasonable. For example, jet production occurs via the processes $q q \rightarrow q q, g g \rightarrow g g, q g \rightarrow q g$, etc. If the measured jet cross-sections are in good agreement with the predicted values, we can have confidence that the distribution functions are reasonable. Such a comparison is shown in Fig. 33. Data from the Spp̄S collider $^{60,61}$ are compared with a prediction using distribution functions extracted 


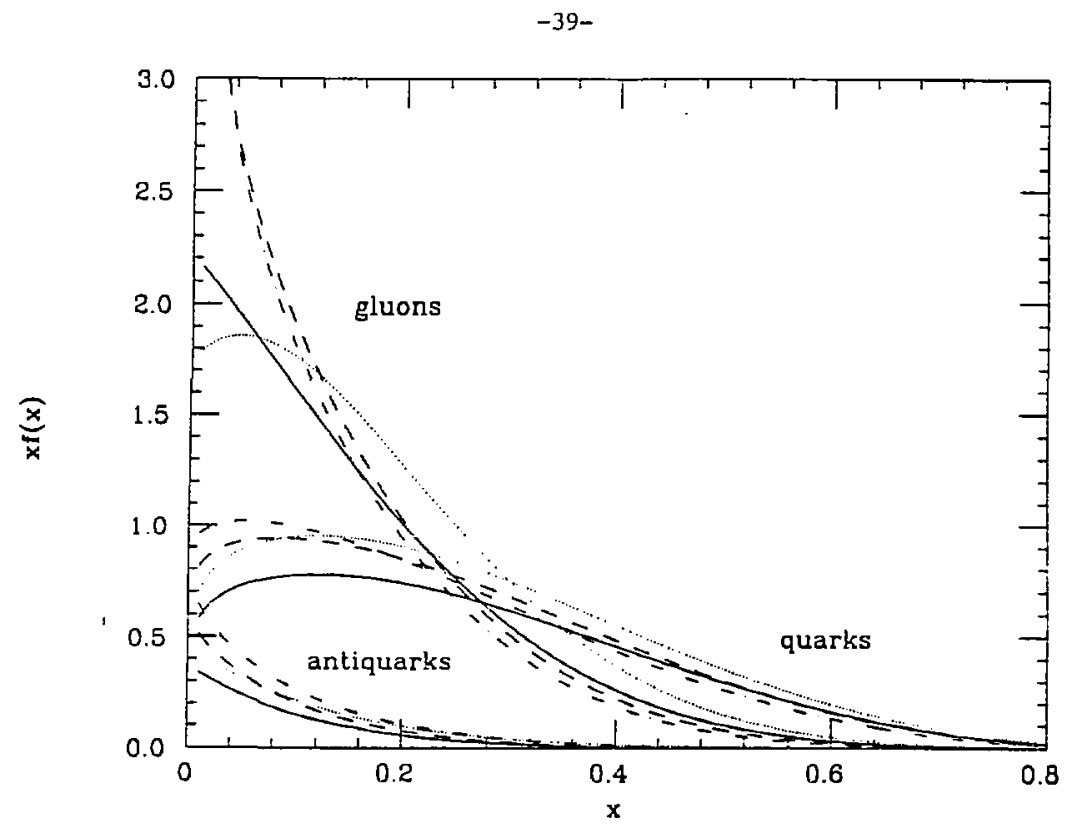

Figure 32: Diagram showing the behavior of the quark and gluon distributions as functions of $x$ for various $Q^{2}$. Plotted is $x f(x)$ for gluons, quarks and antiquarks (summed over quark flavors). The solid (dotted) lines correspond to the structure functions of ref. $58(59)$ at $Q^{2}=5 \mathrm{GeV}^{2}$. The dashed (dot-dashed) lines correspond to the structure functions of ref. 58 (59) at $Q^{2}=25 \mathrm{GeV}^{2}$. The evolution with $Q^{2}$ is given by perturbative $Q C D^{45}$.

from the CDHS neutrino scattering experiment, ${ }^{59}$ which were then extrapolated using $Q C D .{ }^{57}$ Such good agreement leads us to believe that the distribution functions are reliable at the $30 \%$ level.

I will now turn to the question of the scale $Q^{2}$ appearing in $\alpha_{s}\left(Q^{2}\right)$ (Eq. 3.36 and 3.37) and in the distribution functions. Suppose we shift the scale in $\alpha_{0}\left(M^{2}\right)$

$$
\alpha_{s}\left(x M^{2}\right)=\alpha_{s}\left(M^{2}\right)\left[1+\frac{\left(33-2 n_{f}\right)}{12 \pi} \alpha_{s}\left(M^{2}\right) \log x+\mathcal{O}\left(\alpha^{2}\right)\right]
$$

It is therefore clear that we cannot decide the question of scale without computing the order $\alpha_{1}^{3}$ in Eqs. (3.36) and (3.37). A bad choice of scale is likely to result in large $\alpha_{a}^{3}$ corrections. In the absence of such corrections we can only guess what the scale should be. Common sense dictates that it should be of order the quark mass $M_{Q}$. However, if the quark is being produced at large transverse momenta $\left(p_{\perp}\right)$, then something like $\sqrt{M_{Q}^{2}+p_{\perp}^{2}}$ is probably appropriate. 


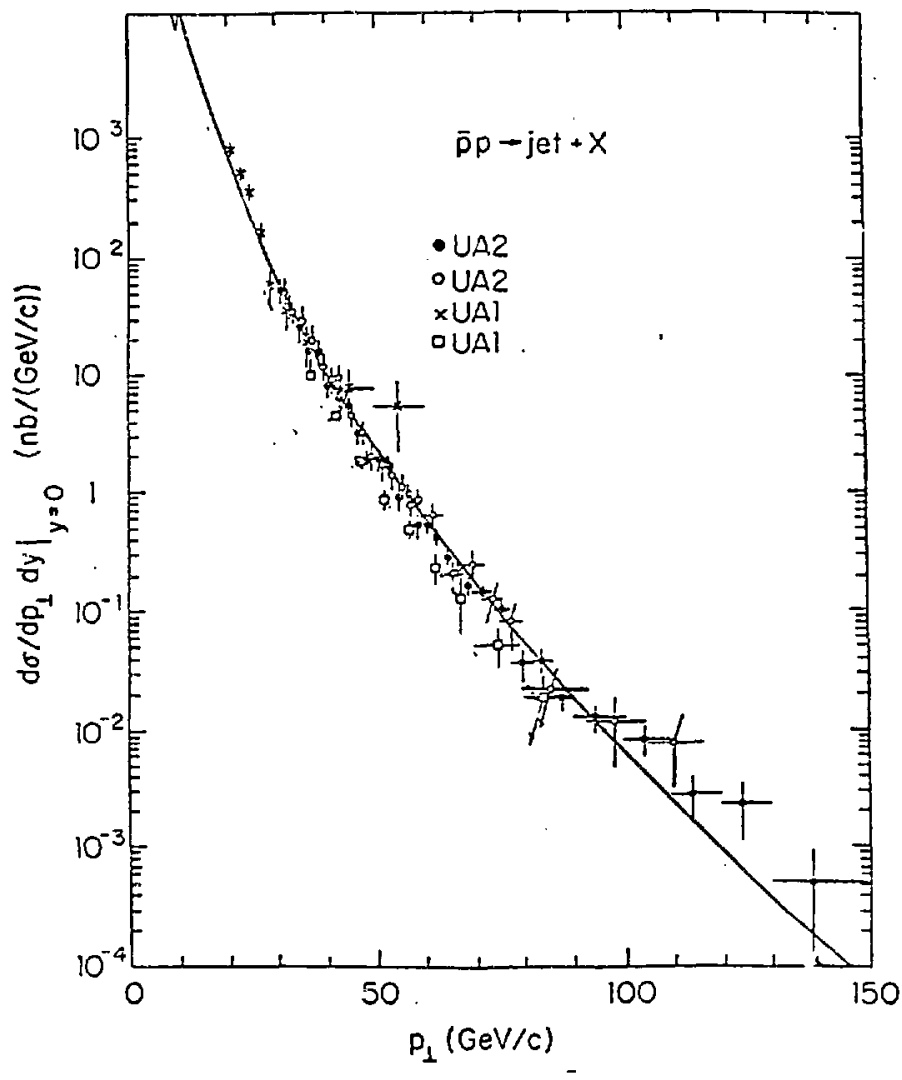

Figure 33: A comparison of the cross-section $p \bar{p} \rightarrow$ jet $+X$ with a calculation using perturbative $Q C D$ for the subprocess $q q \rightarrow q q, g g \rightarrow$ $g g$, etc. The structure functions are those of Ref. 59 evolved in $Q^{2}$ up to the relevant scale $\left(Q=p_{\perp} / 2\right)$. The data are from the UA1 ${ }^{60}$ and $\mathrm{UA2}^{02}$ collaborations.

To claim that the value of $M_{Q}$ introduces an ambiguity may seem absurd. But suppose we are calculating the production rate for charm or bottom quarks; we must decide what value to use. The total production cross-section is a very strong function of $M_{Q}$, it varies roughly as $M_{Q}^{-4}$. What value of the charm quark mass should be used? This question is not easy to answer. The threshold for $c \bar{c}$ production opens when there is sufficient energy in the partonic collisions to produce a $D \bar{D}$ meson pair. This could suggest that one should use $M_{Q}=M_{D}$. However, the quark mass which appears in other calculations, such as that for the energy levels of the $\psi$ system, is usually less than this. The uncertainty induced by $M_{Q}^{f}$ becomes irrelevant for quarks heavier than the $b$. 


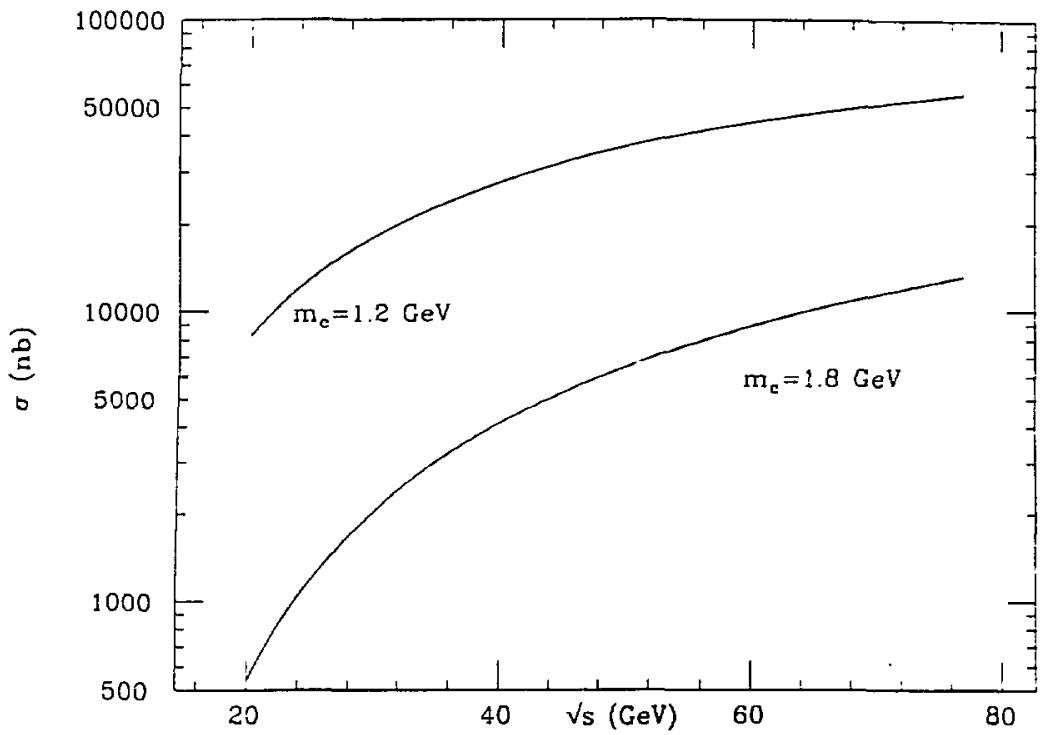

Figure 34: The cross section for the production of a charmed quark pair in proton-proton collisions as a function of $\sqrt{s}$.

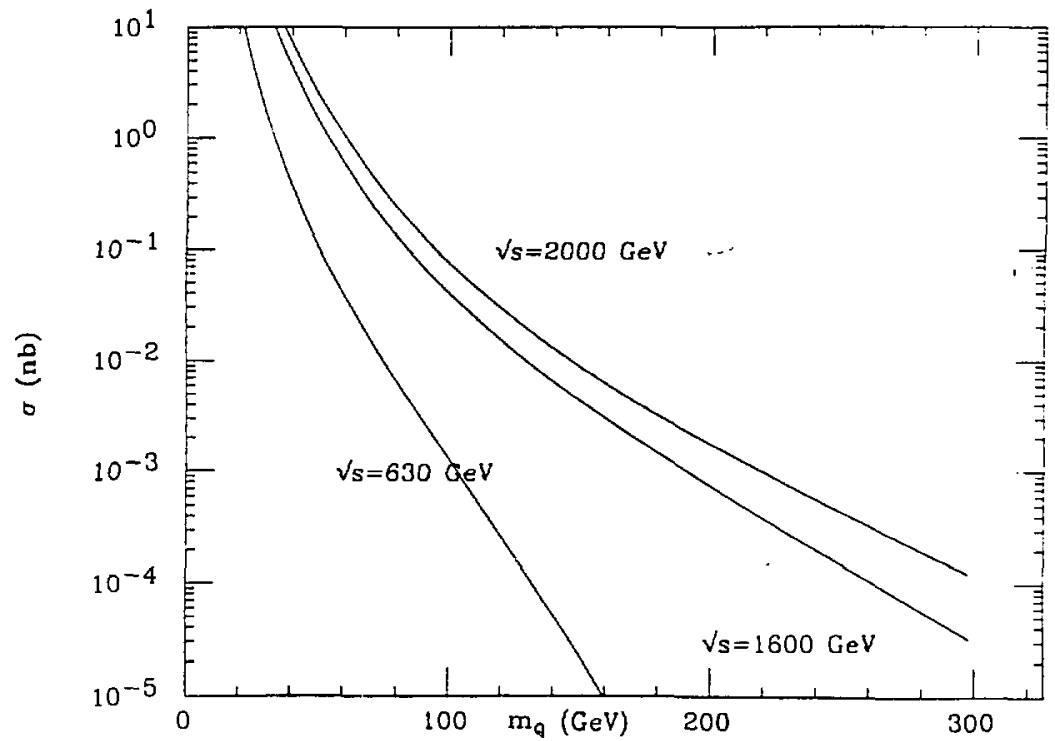

Figure 35: The cross section for the production of a heavy quark pair in proton antiproton collisions as a function of the heavy quark mass. 
Figure 34 shows the total cross-section for the prsduction of charmed quark pairs at small values of $\sqrt{s}$. This figure illustrates the longstanding problem of charm production rates. Cross-sections measured at the $\operatorname{ISR}^{62}(\sqrt{s} \approx 50 \mathrm{GeV})$ usually gave rates which are approximately a factor of 10 larger than $Q C D$ expectations. Mecsirements of $\sqrt{s} \approx 25 \mathrm{GeV}^{63}$ using protons on a stationary target gave results which were closer to $Q C D$ expectations. New results at $\sqrt{s}=43 \mathrm{GeV}^{64}$ are also close to the $Q C D$ values. The ISR experiments had poor acceptance and it is now difficult to reconcile their large values with those obtained at $\sqrt{3}=43 \mathrm{GeV}$. It seems fair to conclude that the $Q C D$ model works reasonably well ${ }^{65}$, and while there may be some need to invoke new mechanisms to explain the rapidity distribution ${ }^{66}$ of the produced charmed particles, no drastic modifications are required.

The cross section for heavy quark production at the $S p \bar{p} S$ colliders is shown in figure 35. If we consider the detetion of heavy quarlis we can be forced into a kinematical regime where the production cross section is not described by the pair production processes. The mean value of the transverse momenta of the quarks produced by the mechanisms discussed above is of order the quark mass. If detection considerations force us to look only at new quarks which are produced at tranvserse momenta which are much larger than this, then other mechanisms can become more important. ${ }^{67}$ If quarks are produced at high transverse momenta via the processes of equations 3.37 and 3.38 then the transverse momenta of the quark and antiquark will balance each other. The process $g+g \rightarrow g+Q+\bar{Q}$ can yield a final state where the quark and antiquark are moving in the same direction, their transverse momenta being balanced by the final state gluon. Although this process is of order $\alpha^{3}$, It can dominate if the transverse momentum is large enough.

\section{The inadequacy of the standard model.}

In the previous sections I have discussed some aspects of the standard model. Despite the lack of experimental data which fails to agree with this model, most theorists find it unsatisfactory. One of the troubling features of this model is the origin of the electroweak scale. The origin of the scale of strong interactions, either $\Lambda$ or the proton mass, can be understood qualitatively as follows. In the context of any unified theory, either a conventional grand unified theory or a more exotic one based on superstrings, all the gauge coupling constants of the standard model are related at some large scale $M$ where the theory is unified. Qualitatively,

$$
\alpha_{\mathrm{em}}\left(M^{2}\right) \approx \alpha_{0}\left(M^{2}\right)
$$

$M$ is of order the Planck mass $\left(M_{P}=10^{1 \theta} \mathrm{GeV}\right)$, or possibly less $\left(\sim 10^{15} \mathrm{GeV}\right)$ in some models. At some scale $Q_{0}, \alpha_{0}\left(Q_{0}\right)$ will become large and $Q C D$ perturbation theory will no longer be valid. At this scale non-perturbative effects will become important and hadronic bound states will form with mass of order $Q_{0}$. Requiring $\alpha\left(Q_{0}\right)=1$ implies that

$$
Q_{0}^{2} \simeq M^{2} \exp \left[\frac{\left(33-2 n_{f}\right)}{12 \pi}\left[1-\frac{1}{\alpha\left(M^{2}\right)}\right]\right]
$$

It is easy to see that for $\alpha\left(M^{2}\right) \approx 1 / 40, Q_{0} / M \approx 10^{-16}$ and the large hierarchy of scales between hadron masses and $M$ can be explained. The presence of the exponential in Eq. (4.2) guarantees that the large ratio $M / Q_{0}$ will be generated. 


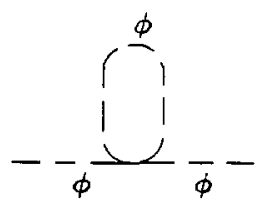

Figure 36: Feynman diagram showing a contribution to the Higgs mass renormalization in the Weinberg-Salam model.

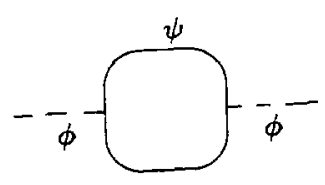

Figure 37: Feynman diagram showing contributions to the mass renormalization of the scalar field $\phi$ (dashed lines) from its self interactions and those with the fermion $\psi$ (solid lines) in the toy model of equation 4.5 .

A similar argument cannot be used to explain the small ratio $M_{W} / M_{P}$. There is a dimensionful parameter $\mu$ in the $S U(2) \times U(1)$ Lagrangian, which is chosen to have a value of order $M_{W}$. Consider a radiative correction to this mass term due to the $\lambda\left(\Phi^{+} \Phi\right)$ interaction (see Fig. 36)

$$
\delta m^{2}=i \lambda \int \frac{d^{4} k}{(2 \pi)^{4}} \frac{1}{\left(k^{2}-\mu^{2}\right)}
$$

This integral is quadratically divergent: cut it of at scale $\Lambda$. Then the value of $\mu^{2}$ computed at one loop is given by

$$
\mu_{1000}^{2}=\mu^{2}+\lambda \Lambda^{2}
$$

In theories such as those described by David Gross ${ }^{3}$ in his lectures, new physics enters at the Planck mass and cuts off the divergence. In this case $\Lambda$ is of order $10^{19} \mathrm{GeV} . \mu_{\text {lloop }}^{2}$ must be of order $M_{W}^{2}$, so that Eq. (4.4) implies that the bare mass $\mu$ must be adjusted to some 18 significant figures. This fine tuning must take place at all orders in perturbation theory. This unnatural fine tuning is usually referred to as the hierarch; problem and is present in theories with quadratic divergences.

This hierarchy problem can be solved in theories where the quadratic divergences are ameliorated on scales of order $M_{W}$. Since these divergences are associated with scalar fields, the scalar sector of the theory must be modified.

One strategy is to introduce some new fields which cancel the divergence. Consider the following toy model consisting of a scalar field $\phi$ and a (two-component) fermion $\psi$.

$$
\mathcal{L}=\partial_{\mu} \phi^{+} \partial_{\mu} \phi-m_{0}^{2} \Phi^{+} \Phi-\bar{\psi}\left(i, \theta-m_{f}\right) \psi+\lambda_{3} \phi^{4}+\lambda_{2} \bar{\psi} \psi \phi
$$

In this theory, there are two one-loop diagrams contributing to the one-loop calculation of the scalar mass (see Figs. 36 and 37). The scalar loop gives

$$
\delta m_{a_{1}}^{2}=i \lambda_{1} \int \frac{d^{4} k}{(2 \pi)^{4}} \frac{1}{\left(k^{2}-m_{e}\right)^{2}}
$$

and the fermion loop, 
If there are no Higgs fields then the $W$ is massless and its propagator (calculated to lowest order in $g_{2}$ ) is

$$
\frac{g^{\mu \nu}-k^{\mu} k^{\nu} / k^{2}}{k^{2}}
$$

The effect of $\Pi^{\mu \nu}$ is to modify the propagator thus:

$$
\frac{g^{\mu \nu}-k^{\mu} k^{v} / k^{2}}{k^{2}\left[1+\frac{9_{2}^{2}}{4} \Pi\left(k^{2}\right)\right]}
$$

Since the lowest lying $U \vec{D}$ bound state is massless $\Pi\left(k^{2}\right)=f_{x} / k^{2}$ as $k^{2} \rightarrow 0$. Hence (4.10) has no pole at $k^{2}=0$ but instead acquires a pole at $M_{W}^{2}=\frac{g_{7}^{2}}{4} f_{\pi}^{2}$. The "pion" is eaten and becomes the extra polarization state of the massive $W$ boson. If this mechanism is to yield the correct value for $M_{W}$, then we need $f_{\pi} \approx 250 \mathrm{GeV}$. The neutral pions cause mixing between the " $W_{3}$ " (i.e., the $I_{3}=0$ member of the $S U(2)_{L}$ gauge boson multiplet) and $B$, the gauge boson of $U(1)_{V}$. The couplings of " $\pi^{0 n}$ to $B$ and $W_{3}$ are determined by the weak charges of the $U$ and $D$ quarks. The resulting mass matrix has the following form:

$$
\frac{f_{\pi}^{2}}{4}\left(\begin{array}{lr}
g_{2}^{2} & g_{2} g_{1} \\
g_{2} g_{1} & g_{1}^{2}
\end{array}\right)
$$

This has one zero eigenvalue corresponding to the photon and one non-zero with mass

$$
M_{Z}^{2}=\left(\frac{g_{1}^{2}+g_{2}^{2}}{4}\right) f_{\pi}^{2}=\frac{M_{W}^{2}\left(g_{1}^{2}+g_{2}^{2}\right)}{g_{2}^{2}}
$$

Notice that this corresponds to $\rho=1$ (see Eq.(1.27)). This theory possesses a custodial $S U(2)$ symmetry since there is a chiral symmetry $S U(2)_{L} \times S U(2)_{R} \times U(1)$ which breaks to $S U(2) \times U(1)$ in the binding.

Such a theory can be expected to have more states than are present in the Weinberg-Salam model. For example, there will be spin-1 bound states $U \bar{D}, D \bar{U}, U \bar{U}+$ $D \bar{D}, U \bar{U}-D \bar{D}$ analogous to the $\rho$ and $\omega$ mesons in $Q C D$. These will have mass of order a few times $f_{\pi}$ and will decay to $W W, W Z$ and $Z Z$ (recall that $\rho \rightarrow 2 \pi$ in $Q C D$ and that the ${ }^{4} \pi^{n}$ in this technicolor theory has been absorbed into the $W$ and $Z$ ). One should also get technibaryons made from $U U U$, etc.

This simple technicolor theory which İ have described cannot explain quark and lepton masses. The electron mass arises in the Weinberg-Salam model from the coupling

$$
\lambda \bar{\Phi} \psi_{L} \psi_{R}
$$

which generates $m_{e}=\lambda(\Phi)$. The analogous term in a technicolor theory would be

$$
\bar{U}_{L} U_{R} \bar{\psi}_{L} \psi_{R}
$$

Such a term is non-renormalizable and is not allowed. The problem is solved by introducing yet another set of interactions which have massive gauge bosons. These bosons mediate interactions between the quarks and techniquarks. The interaction (4.14) then arises as the low energy limit of this theory, in the same way that the Fermi-interaction arises as a low energy limit of the Wainberg-Salam model. Such extended technicolor theories have a rich phenomenology which is discussed in detail in review articles to which the reader is referred. ${ }^{70,72}$ 
e.g.,

$$
J_{\mu}=\bar{\psi} \gamma_{\mu} \gamma_{s} \psi
$$

These currents are connected with the global symmetries and must be conserved at the classical level:

$$
\partial_{\mu} J^{\mu}=0
$$

The explicit calculation of the three-point function of three of these currents ( $\Gamma^{\text {pwa }}$ ) may reveal the presence of an anomaly.

$$
q_{3 \mu} \Gamma^{\mu w a}=A e^{\nu \lambda a \beta} q_{2 \lambda} q_{1 \beta}
$$

with $A$ non-zero, so that the current is no longer conserved. If the three-point function is evaluated at $q_{1}^{2}=q_{2}^{2}=q_{3}^{2}=q^{2}$ it must have the form ${ }^{75}$

$$
\Gamma^{\mu \nu \lambda}=\frac{1}{q^{2}} A\left[e^{\mu \alpha \beta} q_{1}^{\alpha} q_{2}^{\beta} q_{3}^{\lambda}+\text { permutations }\right]+\text { terms finite as } q^{2} \rightarrow 0
$$

If the chiral symmetries are described by some group $G$ and the preons are in a representation $R$ with representation matrices $\left(\lambda^{a}\right)_{i j}$, then the currents $\left(J_{\mu}^{a}\right)$ contained in the $R \times \bar{R}$ representation. In particular, they can be in the adjoint representation. The coefficient $A$ is proportional to $\operatorname{Tr}\left[\lambda^{a} \lambda^{b} \lambda^{c}\right]$.

If the momentum scale $\sqrt{q^{2}}$ of the probing currents is much less than the scale $\Lambda$ of the preonic binding, then the relevant spectrum of states is not that of the preons but rather that of the bound states. It follows that if the asomaly is calculated using the bound states, the same result must be obtained. In particular, the bound states must reproduce the singularity at $q^{2}=0$ in Eq. (4.18). This can be done in one of two ways.

The chiral symmetry could be spontaneously broken by the binding. In this case there must be a massless Goldstone boson which is coupled to the currents as indicated by Fig. 40. The boson propagator yields a factor of $1 /\left(q^{2}-m_{\text {bosom }}^{2}\right)$, which generates the correct dependence on $q^{2}$ at small $q^{2}$ since $m_{\text {boson }}$ is zero.

If the chiral symmetry is maintained by the binding, there will be massless bound state fermions. These fermions, when inserted in the loop of Fig. 39, can generate a contribution which has the correct $q^{2}$ dependence. Notice that the massive states bound states (of mass $M$ ) are not relevant since they will generate terms of order

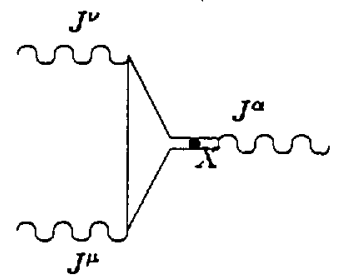

Figure 40: Diagram showing the contribution of a massless fermionic bound state $(X)$ to the three point function of Eqn 4.18. 
$1 / M^{2}$ at small $q^{2}$. The value of $A$ will depend upon the number of such massless bound states and their coupling, which is determined by the symmetry. Hence it may not be possible to get the same value of $A$ from the massless bound states as from the preons. If the anomalies do not match then the chiral symmetry must be broken in the binding and there will be no massless fermions.

This matching condition is very difficult to satisfy. Let us take the familiar case of $Q C D$ as an example. Assume that there are only two massless quarks (the up and down). The quark theory now has a global symmetry of the type

$$
S U(2)_{L} \times S U(2)_{R} \times U(1)
$$

The subscripts $L$ and $R$ refer to the fermion helicity states. A linear combination of these $S U(2)$ 's is the familiar strong isospin. The quarks are in the 2 representation of each of the $S U(2)$ 's. The bound states must be singlets of $S U(3)_{\text {ode }}$ which contain three quarks. These bound states must transform according to the $2 \times \underline{2} \times \mathbf{3}$ representation. It is therefore clear that the anomaly calculated from the quarks (a doublet) can match that calculated from the bound states consisting of a single doublet. It is therefore possible for the ground state of the theory to have two massless fermions.

Let us contrast this with what happens if there are three massless flavors. In this case, the global symmetry is

$$
S U(3)_{L} \times S U(3)_{R} \times U(1)
$$

The fermionic bound states must again contain three quarks and must therefore be contained in the $3 \times 3 \times 3$ representation of the global $S U(3)$. The possibilities are 10,8 and 1 . None of these generates the same anomaly as the $\underline{3}$, so that there cannot be any massless composites and the chiral symmetry must be spontaneously broken in the binding. In the real case of $Q C D$ the strange quark is light; it may be this fact which forces chiral symmetry to be broken.

Notice that the consistency conditions cannot predict whether or not the chiral symmetry is unbroieis if they are satisfied. Dynamical considerations may still favor the phase where it is broken even if the conditions are satisfied.

The second mechanism for providing massless bound states is the quasi-Goldstone fermion method. ${ }^{76}$ The spontaneous breaking of a continuous symmetry will give rise to massless Goldstone bosons. If the theory has an unbroken supersymmetry these bosons must have degenerate fermionic partners. If a supersymmetric theory can be arranged so that during the binding process such a global symmetry is broken, then there will be massless fermionic bound states. Some toy models that have been constructed have both protection mechanisms for the fermion masses. 7

Even after a model of massless fermions has been constructed there remains a potentially more serious problem. The quarks and leptons have small masses. A way must be found to generate this mass without destroying the protection and so promoting the masses up to the scale of binding. This can be done in the chiral models by giving the preons a small mass (much less than $\Lambda$ ) so that although the chiral symmetry is not exact, it is a very good approximation. The ground state will now consist of almost massless bosons or almost massless composite fermions. The former case arises in $Q C D$ where the small quark masses give rise to a non-zero pion mass. 
In the pseudo-Goldstone fermion models the problem is more difficult. There are no light elementary scalars, so that as well as generating a small quark and lepton mass, the scalars must be given masses in excess of $20 \mathrm{GeV}$ or so.

There is only one model of composite quarks and leptons which is realistic. This is a variant of the Weinberg-Salam model in which the weak interactions are assumed to be confining. ${ }^{78}$ The preons consist of the usual matter representations, fermions which I will call pre-leptons and pre-quarks and scalar Higgs bosons. The ground state is assumed to be that where the Higgs vacuum expectation values is zero and the $S U(2)_{L}$ interactions are taken to be confining on a scale $\Lambda$ of order $100 \mathrm{GeV}$. The candidates for massless fermionic bound states consist of a scalar and a prequark or pre-lepton. These states are singlets with respect to $S U(2)_{L}$ and can be shown to satisfy the consistency conditions. These bound states are the usual left-handed quarks and leptons. The right-handed quarks are elementary, being identical to those in the standard model. (They are singlets of $S U(2)_{L}$ and are therefore not bound.)

If this binding occurs one should expect the existence of spin- 1 and spin-0 bound states made of elementary scalars. Such states should have mass of order $\Lambda$. These states play the role of the physical Higgs and the $W$ and $Z$ bosons of the standard model. The form of the coupling of these states to the quarks and leptons is the same as that in the standard model. This model is not inconsistent with any data, nevertheless it has some features which make me uncomfortable."

The coupling between a $W$ and a quark is known and is small. It seems odd that two bound states should couple so weakly. One would also expect a large number of massive states with masses of the same order as the $W$ boson. In order to reconcile the model with the data the mass of these states must exceed $120 \mathrm{GeV}$ or so. The degeneracy in this model between the $W$ and $Z$ mass is lifted by electromagnetic corrections which mix the $Z$ and the photon. It is rather difficult to imagine this mixing being large enough to generate the observed mass splitting between the $W$ and $Z$ bosons.

Fortunately, it is possible to analyze the phenomenological consequences of compositeness without reference to a specific model. This is done by placing phenomenological constraints on the possible effective operators ${ }^{80}$ which involve quarks and leptons and which an arise as a result of the interactions which are responsible for the binding. In the strongly coupled Weinberg-Salam model, it is one of these operators which plays the role of the Fermi coupling.

One of the most accurately known quantities in high energy physics is the anomalous magnetic moment of the muon. A theory in which the muon is composite is likely to have more massive states with the same quantum numbers (called $\mu^{\circ}$ ). An interaction is possible involving the photon, the muon and the excited muon, ${ }^{82}$ viz.,

$$
\frac{e}{2 \Lambda} \bar{\mu} \sigma^{\mu \nu}\left(a-b_{\tau}\right) \mu \cdot F_{\mu \nu}
$$

I have included a factor of e since the coupling is electromagnetic. The appropriate power of $\Lambda$. is determined by dimensional analysis.

The parameter $\Lambda$, is of order the scale of the binding of the muon constituents. Its exact value is model dependent. Such an interaction will give a contribution to 
$(g-2)$ at one loop shown in Fig. 41.

$$
\delta(g-2)=m_{\mu} \frac{\left(a^{2}-b^{2}\right)}{\Lambda}+\frac{\left(a^{2}+b^{2}\right) m_{\mu}^{2}}{\Lambda^{2}}
$$

In making this estimate, it has been assumed that the mass of $\mu^{*}$ is of order $\Lambda$. If the underlying preonic theory is chiral, as it must be if chiral symmetry is used to guarantee the appearance of light bound states, $a=b$ and the contribution to $(g-2)$ is suppressed by $m_{\mu}^{2} / \Lambda^{2}$. The constraint on $\Lambda$ is rather poor

$$
\Lambda \gtrsim 100 \mathrm{GeV}
$$

Instead of writing an interaction involving $\mu^{*}$, I could have written an interaction involving only the muon, viz.,

$$
\frac{m_{\mu}}{\Lambda^{2}} \bar{\mu} \sigma^{\mu \nu} \mu F_{a \rho}
$$

Again, $\Lambda$. is of the order of the composite scale; it has been assumed that the binding forces are chiral, hence the factor of $m_{\mu}$.

I shall analyze the remaining consequences of compositeness in this manner so that I shall not have to make reference to the more massive states, such as the $\mu^{*}$.

It is possible that the muon and electron could share constituents, in which case processes like $\mu \rightarrow$ er will be expected to occur. Such transitions will be suppressed by powers of $\Lambda .{ }^{\dagger}$ The lowest dimension operator which can contribute to the decay process will be the one which dominates unless it has a very small coefficient. In this case the operator is a four-fermion operator

$$
\frac{g^{* 2}}{\Lambda^{* 2}} \bar{\mu} \gamma^{a} \frac{\left(1-\gamma_{5}\right)}{2} e \bar{e} \gamma^{a} \frac{\left(1-\gamma_{5}\right)}{2} e+(\mu \mapsto e)
$$

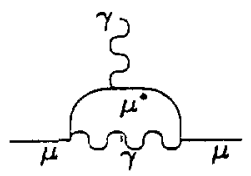

Figure 41: A contribution to $(g-2)$ of the muon from the interaction of muons, excited muons and photons of Eqn. 4.19.

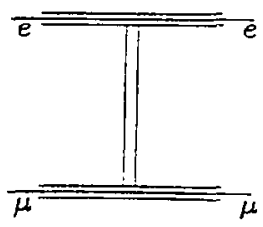

Figure 42: Diagram showing the origin of the four-fermion operator of Eqn. 4.23, due to the exchange of some exotic state with mass of or$\operatorname{der} \Lambda^{*}$.

\footnotetext{
'Reeall that in a grand unified theory proton decay is mediated by interactions which operate on the scale of grand unification and hence the proton decay rate is suppressed by powers of this large scale
} 
In this expression I have inserted a factor of $g^{* 2}$, where $g^{*}$ is the coupling constant of the composite theory. The effective operator (4.23) arises from a diagram of the type indicated in Fig. 42. The diagram involves the exchange of some particle of mass order $\Lambda$. with coupling of order $g^{*}$. Again, the exact value will depend on the particular model. Since the binding is expected to occur at strong coupling, it is usual to take $g^{* 2}=4 \pi$ so that $\alpha_{*}=g^{* 2} / 4 \pi=1$. Notice that this is not the case in the composite model discussed above where the $W$ and $Z$ are composite. In that model the coupling is $g_{W} \approx 0.68$.

The operator of Eq. (4.23) can also be bounded from the failure to observe the process $\mu \rightarrow 3 e$. There is one other lepton number violating operator which can occur

\begin{tabular}{|c|c|c|}
\hline Process & Contact Interaction & $\begin{array}{c}\text { Limit on } \Lambda^{*} \\
(\mathrm{TeV})\end{array}$ \\
\hline$(g-2)$ & $\frac{m_{r}}{\Lambda_{l}^{?}} \bar{e} \sigma^{\alpha \beta} e F_{\alpha \beta}$ & .03 \\
\hline$(g-2)_{\mu}$ & $\frac{m_{1}}{A_{1}^{\frac{1}{2}}} \bar{\mu} \sigma^{\alpha \beta} \mu F_{\alpha \beta}$ & .86 \\
\hline$\mu \rightarrow e \gamma$ & $\frac{4 \pi}{\Lambda_{\bar{l}}^{\mu}} \bar{\alpha} \gamma^{\alpha} \frac{1}{2}\left(1-\gamma_{s}\right) e \bar{e} \gamma_{\alpha} \frac{1}{2}\left(1-\gamma_{s}\right) e+(\mu \leftrightarrow e)$ & 60 \\
\hline$\mu \rightarrow 3 e$ & $\frac{4 \pi}{\Lambda^{2}} \bar{\mu} \gamma^{\alpha} \frac{1}{2}\left(1-\gamma_{s}\right) e \bar{e} \gamma_{\alpha} \frac{1}{2}\left(1-\gamma_{s}\right) e$ & 400 \\
\hline$\mu \mathrm{N} \rightarrow \mathrm{eN}$ & $\frac{4 \pi}{\Lambda^{2}} \bar{\mu} \gamma^{\alpha} \frac{1}{2}\left(1-\gamma_{s}\right) e \bar{d} \gamma_{\alpha} \frac{1}{2}\left(1-\gamma_{3}\right) d$ & $\$ 60$ \\
\hline$K_{L} \rightarrow e^{ \pm} \mu^{\mp}$ & $\frac{4 \pi}{\hat{n} !} \bar{s} \gamma^{a} \frac{1}{2}\left(1-\gamma_{5}\right) u \bar{e} \gamma_{\alpha} \frac{1}{2}\left(1-\gamma_{5}\right) \mu$ & 140 \\
\hline$K^{+} \rightarrow \pi^{+} e^{-} \mu^{+}$ & $\frac{4 \pi}{\Lambda^{3}} \overline{3} \gamma^{\alpha} \frac{1}{2}\left(1-\gamma_{5}\right) u \bar{e} \gamma_{\alpha} \frac{1}{2}\left(1-\gamma_{5}\right) \mu$ & 210 \\
\hline$\Delta M\left(K_{L}-K_{s}\right)$ & $\frac{4 \pi}{\Lambda} \bar{s} \bar{s} \gamma^{a} \frac{1}{2}\left(1-\gamma_{5}\right) d \bar{s} \gamma_{a} \frac{1}{2}\left(1-\gamma_{5}\right) d$ & 6100 \\
\hline
\end{tabular}

Table 5: Limits of contact terms from rare processes. The interaction type assumed for each rare process is shown along with the resulting limit on the compositeness scale $\Lambda^{*}$. 
where $q$ is a quark. Such an operator could contribute to the deep inelastic scattering process $\mu+$ nucleon $\rightarrow e+$ hadrons. Such processes have not been observed. The bound on $\Lambda$ arising from such lepton number violating processes is shown in Table 4 .

The interactions involving quarks may give rise to flavor-changing neutral currents. The experimental constraints on the absence of such currents are very strong. A $\Delta S=2$ transition mediated by the operator

$$
\frac{g^{-2}}{\lambda^{2}} \bar{s} \gamma^{\alpha} \frac{\left(1-\gamma_{5}\right)}{2} d \bar{s} \gamma_{a} \frac{\left(1-\gamma_{5}\right)}{2} d
$$

will give a contribution to the $K_{L}-K_{S}$ mass difference. The mass difference arises in the standard model from the second order weak process involving the exchange of two $W$ bosons. If such a new contribution is present, $A$. must be larger than $6 \mathrm{TeV}$ ! Table 5 extracted from the lectures of Eichten ${ }^{82}$ gives a summary of the compositeness limit arising from the absence of rare processes. These limits are given assuming that $g^{\bullet 2}=4 \pi$. They are less stringent in models where the composite states are weally coupled.

If the composite interactions do not yield flavor changing interactions between quarks and leptons, then the limits from the indirect effects of compositeness are rather weak. In this case one must turn to more direct searches. In the case of composite electrons, one expects that there will be a four electron operator of the form $^{80}$

$$
\begin{aligned}
g^{.2} / 2 \Lambda_{\cdot}^{2} \quad & {\left[\eta_{L L} \bar{e} \gamma^{u} \frac{\left(1-\gamma_{5}\right)}{2} e \bar{e} \gamma^{u} \frac{\left(1-\gamma_{5}\right)}{2} e\right.} \\
& \eta_{R R} \bar{e} \gamma^{u} \frac{\left(1+\gamma_{5}\right)}{2} e \bar{e} \gamma^{u} \frac{\left(1+\gamma_{5}\right)}{2} e \\
& \left.2 \eta_{L R} \bar{e} \gamma^{u} \frac{\left(1-\gamma_{5}\right)}{2} e \bar{e} \gamma^{u} \frac{\left(1-\gamma_{5}\right)}{2} e\right]
\end{aligned}
$$

This term will interfere with the usual one-photon exchange process and cause a modification of the Bhabhascattering cross-section $e^{+} e^{-} \rightarrow e^{+} e^{-}, v i z$,

$$
\begin{aligned}
& \frac{d \sigma}{d \Omega}=\frac{\pi \alpha^{2}}{4 s}\left[4 A_{0}+A_{+}(1+\cos \theta)^{2}+A_{-}(1-\cos \theta)^{2}\right] \\
& A_{0}=\left(\frac{s}{t}\right)^{2}\left|1+\frac{g_{R} g_{L}}{e^{2}} \frac{t}{t_{z}}+\frac{\eta_{R L} t}{\alpha \Lambda_{0}^{2}}\right|^{2}, \quad A_{-}=\left|1+\frac{g_{R} g_{L}}{e^{2}} \frac{s}{s_{z}}+\frac{\eta_{R L} s}{\alpha \Lambda_{0}^{2}}\right|^{2} . \\
& A_{+}=\frac{1}{2}\left|1+\frac{s}{t}+\frac{g_{R}^{2}}{e^{2}}\left(\frac{s}{s_{x}}+\frac{s}{t_{z}}\right)+\frac{2 \eta_{R R} g}{\alpha \Lambda_{t}^{2}}\right|^{2}+\frac{1}{2}\left|1+\frac{s}{t}+\frac{g_{L}^{2}}{e^{2}}\left(\frac{s}{s_{x}}+\frac{s}{t_{z}}\right)+\frac{\left.2 \eta_{L L}\right|^{2}}{\alpha \Lambda^{2}}\right|^{2}
\end{aligned}
$$

Here $t=-s(1-\cos \theta) / 2, s_{z}=s-M_{x}^{2}+i \Gamma_{z} M_{z}, t_{x}-t-M_{x}^{2}-i M_{x} M_{z}, g_{R}=e \tan \theta_{w}$ and $g_{L}=-e \cot 2 \theta_{w}$. In this formula I have set $g^{\cdot 2}=4 \pi$, a reasonable value for a strongly coupled theory. Current data can be used to set a limit of $\Lambda .52 .5 \mathrm{TeV} .{ }^{83}$

In the case of quark compositeness an effect will be seen in the production of jets in a hadron collider since one of the relevant partonic processes is quark-quark scattering. The presence of a four-quark operator of the form (4.26) with a quark replacing an electron will yield a modification of the predicted jet cross-section in hadronic collisions. If we take $\eta_{L R}=\eta_{R R}=0$ then this interaction leads to a modification of the cross-section for quark-quark scattering 


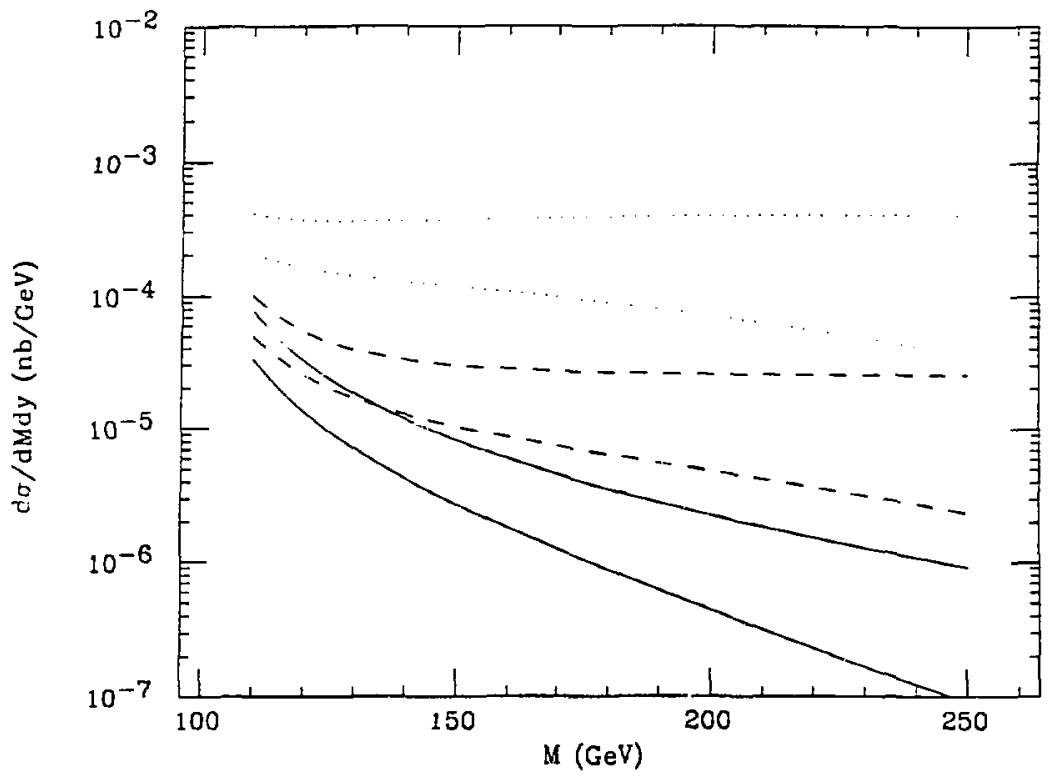

Figure 43: The cross section $d \sigma / d p_{\mathrm{t}} d y$ for the production of jets of hadrons transverse meomentum $p_{\mathrm{t}}$ and rapidity $y=0$. The curves include the effects of compositeness given by Eqn. 4.28 with $\eta L L=1$ and $\Lambda^{\circ}=300 \mathrm{GeV}$ (dotted lines), $1000 \mathrm{GeV}$ (dashed lines) and $5000 \mathrm{GeV}$ (solid lines). The curves are for protonantiproton interactions as $\sqrt{s}=630 \mathrm{GeV}$ (lower lines) and $2000 \mathrm{TeV}$.

$$
\begin{aligned}
\frac{d \sigma}{d t}\left(q_{i} q_{i} \rightarrow q_{i} q_{i}\right) & =\frac{4 \alpha_{s}^{2}}{9 s^{2}}\left[\frac{u^{2}+s^{2}}{t^{2}}+\frac{s^{2}+t^{2}}{u^{2}}-\frac{2}{3} \frac{u^{2}}{s t}\right] \\
& +\frac{8 \eta_{L L} \alpha_{s}}{9 \Lambda_{i}^{2}}\left(\frac{s^{2}}{t}+\frac{s^{2}}{u}\right) \\
& +\frac{\eta_{L L}^{2}}{\Lambda_{i}^{4}}\left(u^{2}+t^{2}+\frac{2 s^{2}}{3}\right) \\
\frac{d \sigma}{d t}\left(q_{i} q_{j} \rightarrow q_{i} q_{j}\right) & =\frac{4}{9} \frac{\pi \alpha_{j}^{2}}{s^{2}}\left[\frac{u^{2}+s^{2}}{t^{2}}+\frac{9}{4 \alpha_{i}^{2}} \frac{\eta_{L L}^{2} u^{2}}{\Lambda^{4}}\right]
\end{aligned}
$$

Again I have taken $g^{\cdot 2}=4 \pi$. It is to be remarked that these effects are not visible in quark-quark scattering until a higher value of $s$ than was the case for Bhabha scattering. This is simply because the dominant contribution to the jet cross-sections arises from gluon-gluon and gluon-quark scattering which are not modified since the gluon is not composite. The effects of compositeness are not visible until $s / \Lambda_{\text {a }}^{2}$ is sufficiently large for the composite effects to raise the quark-quark scattering rates above those for gluon-gluon and gluon-quark. The effect is shown in Fig. 43. Current data from the $S_{p} \bar{p} S$ collider place a limit of order $450 \mathrm{GeV}^{84}$ 
If quarks and leptons share constituents then operators of the type

$$
\eta_{0} \frac{g_{*}^{2}}{4 \pi} \gamma^{u} \frac{\left(1-\gamma_{5}\right)}{2} q \bar{e} \gamma^{u} \frac{\left(1-\gamma_{5}\right)}{2} e
$$

The effect of such a term can be seen in two different ways. First, it will affect the production rate for lepton pairs in a hadron-hadron collision. This is controlled by the process $q \bar{q} \rightarrow e^{+} e^{-}$for which the cross-section has the form

$$
\frac{d \sigma}{d t}\left(q_{i} \bar{q}_{i} \rightarrow l^{+} l^{-}\right)=\frac{\pi \alpha^{2}}{s^{2}}\left[A_{i}(s)\left[\frac{u}{s}\right]^{2}+B_{i}(s)\left[\frac{t}{s}\right]^{2}\right]
$$

where the quark flavors are $i=$ up, down. The coefficients $A_{i}$ and $B_{i}$ may be written as

$$
\begin{aligned}
A_{i}(s) & =\left|Q_{i}-\frac{L_{i} L_{i}}{4 x_{W}\left(1-x_{W}\right)} \frac{s}{s-M_{Z}^{2}+i M_{Z} \Gamma_{Z}}-\frac{\eta_{0} s}{\alpha \Lambda^{-2}}\right|^{2} \\
& +\left|Q_{i}-\frac{R_{i} R_{i}}{4 x_{W}\left(1-x_{W}\right)} \frac{s}{s-M_{Z}^{2}+i M_{Z} \Gamma_{Z}}\right|^{2} \\
B_{i}(s) & =\left|Q_{i}-\frac{R_{i} L_{i}}{4 x_{W}\left(1-x_{W}\right)} \frac{s}{s-M_{Z}^{2}+i M_{Z} \Gamma_{Z}}\right|^{2} \\
& +\left|Q_{i}-\frac{L_{i} R_{i}}{4 x_{W}\left(1-x_{W}\right)} \frac{s}{s-M_{Z}^{2}+i M_{Z} \Gamma_{Z}}\right|^{2}
\end{aligned}
$$

where the chiral couplings of the neutral weak current, are, as usual, $L_{i}=\tau_{3}-2 Q_{i} x_{W}$ and $R_{i}=-2 Q_{i} x W$. Here the weak mixing parameter is $x_{W}=\sin ^{2} \theta_{W}$ and $\tau_{3}$ is twice the weak-isospin projection of fermion $i$.

The cross-sections $d \sigma /\left.d M d y\right|_{y=0}$ for the reaction

$$
p \bar{p} \rightarrow l^{+} l^{-}+\text {anything }
$$

are shown in Fig 44. A contribution of the type (4.29) will also shown up in deep inelastic scattering where it will cause a modification of the oroton structure function $F_{2}\left(x, Q^{2}\right)$. This is discussed in the lectures by Gunter Wolf. ${ }^{3 i}$ The values of $\Lambda$ which can be probed at HERA are similar to those reached using the Dreli-Yan mechanism at the Tevatron collider.

\section{Acknowledgments}

The work was supported by the Director, Office of Energy Research, Office of High Energy Physics, Division of High Energy Physics of the U.S. Department of Energy under Contract DE-AC03-76SF00098. 


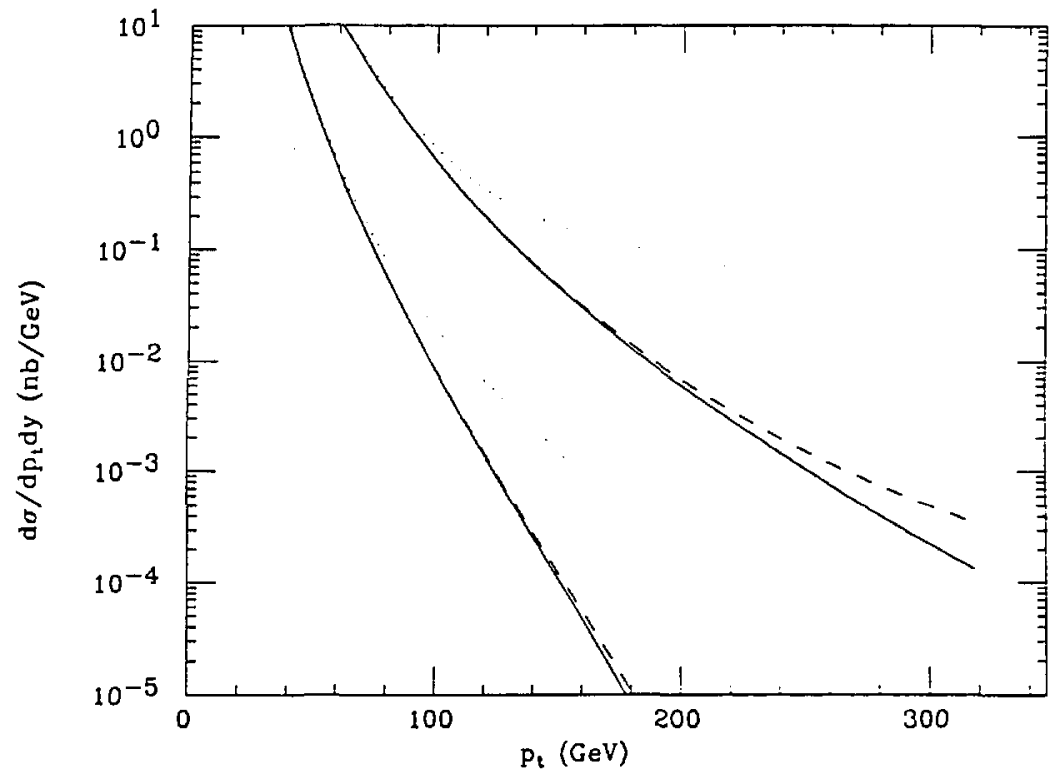

Figure 44: The cross section $d \sigma / d M d y$ for the production of a Drell-Yan pair of mass $M$ and rapidity $y=0$. The curves include the effects of compositeness given by Eqn. 4.31 with $\eta=1$ and $\Lambda^{*}=500 \mathrm{GeV}$ (dotted lines), $1000 \mathrm{GeV}$ (dathed lines) and $5000 \mathrm{GeV}$ (solid lines). The curves are for proton-antiproton interactions as $\sqrt{s}=630 \mathrm{GeV}$ (lower lines) and $2000 \mathrm{TeV}$.

\section{REFERENCES}

1 S. Glashow, Nucl. Phys. 22:579 (1961);

S. Weinberg, Phys. Rev. Lett. 19:1264 (1967);

A. Salam, in: "Elementary Particle Theory," W. Svartholm, ed., Almquist and Wiksell, Stockholm (1968).

2 L. Di Lella, lectures at this school.

3 D. Gioss, lectures at this school;

A. Savoy-Navarro, lectures at this school.

4 Physics at LEP, J. Ellis and R. Peccei, eds., CERN 86-02 (1986).

5 Proceedings of the SSC Workshop, SLAC-PUB-267 (1982).

6 G. 't Hooft and M. Veltman, Nucl. Phys. B 44:189 (1972);

C.G. Bollini, J.J. Giambiagi and A. Gonzáles Dominguez, Nuovo Cim. 31:551 (1964);

J. Ashmore, Nuovo Cim. Lett. 4:289 (1972);

G.N. Cicuta and E. Montaldi, Nuovo Cim. Lett. 4:329 (1972). 
$7 \quad$ W.A. Bardeen et al., Phys. Rev. D 18:3998 (1978).

8 See, for example, J.D. Bjorken and S.D. Drell, "Relativistic Quantum Mechanics," McGraw-Hill (1964).

9 E.R. Williams and P.T. Olsen, Phys. Rev. Lett. 62:1575 (1979).

10 W. Marciano, Phys. Rev. $D$ 20:276 (197S).

11 A. Sirlin, Phys. Rev. $D$ 22:971 (1980).

12 W. Marciano and A. Sirlin, Phys. Rev. $D$ 29:945 (1984).

13 Ch. Llewellyn Smith and J. Wheaten, Phys. Lett. B 105:486 (1981).

14 G. Arnison et al., Phys. Lett. B 166:484 (1986);

J. Appel et al., Z. Physik C 30:1 (1986).

15 L. S. Durkin and P. Langecker, Phys. Lett. B 166:436 (1986).

16 L. DiLella, in "Proceedings of the 1985 International Symposium on Lepton and Photon Interactions at High Energies," Kyoto, Japan (1985).

17 L. A. Abrens et al., Univ. of Penn. preprint E-734-85-1 (1985).

18 C. Prescott et al., P.hys. Lett. B 84:524 (1979);

J.E. et al., Rev. Mad. Phys. 53:211 (1981).

19 F. Bergsma et al., Phys. Lett. B 147:481 (1984);

L.A. Ahrens et al., Phys. Rev. Lett. 54:18 (1985).

M.A. Bouchiat et al., Phys. Lett. B 134:465 (1984);

E.W. Fortson and L.L. Lewis, Phys. Rep. 113:289 (1984).

21 H. Abramowicz et. al, Phys. Rev. Lett. 57:298 (1986);

J.V. Allaby et al., CERN preprint CERN-EP-86/94 (1986).

22 W. Marciano and A. Sirlin, Nucl. Phys. B 189:442 (1981).

23 M.S. Chanouritz, M. Furman and I. Hinchliffe, Nucl. Phys. B 153:402 (1979).

24 M. Veltman, Phys. Lett. B 70:253 (1977).

25 T. Appelquist and J. Carrazone, Phys. Rev. D 11:2856 (1975).

26 S. Weinberg, Phys. Rev. D 19:1277 (1979);

L. Susskind, Phys. Rev. D 20:2619 (1979).

27 G. Barbiellini et al., in Ref. 4.

28 B. Pifer et al., D0 Design Report.

29 B.W. Lynn, M. Peskin and R.G. Stuart, in Ref. 4.

30 D. Blockus et al., "Proposal for Polarization at the SSC."

31 B.W. Lynn and M. Cretic, SLAC-PUB-3900 (1986);

F. Gilman and P. Franzini, SLAC-PUB-3932 (1986).

32 S. Coleman and E. Weinberg, Phys. Rev. $D$ 7:1888 (1973).

33 S. Weinberg Phys. Rev. Lett. 36:294 (1976);

A. Linde, JETP Lett. 23:64 (1976).

A. Guth and E. Weinberg, Phys. Rev. Lett. 65:1131 (1980).

35 R. Flores and M. Sher, Ann. Phys. 168:95 (1983).

36 C. Quigg, D.W. Lee and H. Thacker, Phys. Rev. D 16:1519 (1977).

37 M. Veltman, Acta Phys. Polon. B 8:475 (1977). 
J.D. Bjorken, in: "Proceedings of the 1976 SLAC Summer Institute," M. Zipf, ed.

R.N. Cahn, M.S. Chanowitz and N. Fleishon, Phys. Lett. B 82:113 (1979).
B.L. Ioffe and V.A. Khoze, Leningrad report LINP-274 (1976).

F. Wilczek, Phys. Rev. Lett. 39:1304 (197i).

M. Vysotsky, Phys. Lett. B 97:159 (1980).

H.M. Georgi et al., Phys. Rev. Lett. 40:692 (1978).

R.N. Cahn and S. Dawson, Phys. Lett. B 136:196 (1986);

S. Petcov and D.R.T. Jones, Phys. Lett. $B$ 84:660 (1979).

M. Chanowitz and M.K. Gaillard, Phys. Lett. $B$ 142:85 (1986).

Z. Kunszt, Nucl. Phys. B 247:339 (1984).

J.F. Guaion et al., Phys. Rev. Lett. 54:1226 (1985).

E. Eichten et al., Rev. Mod. Phys. 56:579 (1986).

J.F. Gunion and M. Soldate, Phys. Rev. D 34:826 (1986).

J. Ellis, M.K. Gaillard and D.V. Nanopoulos, Nucl. Phys. B 106:292 (1976).

G. Kane University of Michigan preprint UM TH 86-16 (1986), F. Gilman, J. Gunion and P. Wiesman, In preparation.

G. Altarelli Phys. Rep. 81:1 (1982).

A. H. Mueller Lectures at the 1985 TASI (yale University), published by Wordl Scientific Publishing (Singapore).

M. Dize and J. Saperstein Phys. Rev. Lett. 43:688 (1979), W Celmaster and R J. Gonsalves Phys. Rev. Lett. 44:560 (1979).

G. Altarelli and G. Parisi, Nucl. Phys. B126:298 (1977).

G. Altazelli, R. K. Ellis and G. Martinelli, Nucl. Phys. B143 521 (1978).

B. L. Combridge, Nucl. Phys. B151:429 (1977).

F. Bergsma et al., Phys. Lett. 123B:269 (1983).

H. Abramowicz et al., Z. Physik C13:199 (1982), C17:283 (1983).

P. Bagnaia et al., Z. Physik C20:117 (1983),

G. Arnison et al., Phys. Lett. 123B:115 (1983),

D. Drijard et al., Phys. Lett. 85B:425 (1979).

R. Ammer et al., Phys. Lett. 135B:237 (1984).

R. Ammer et al., CERN EP/86-122 (1986).

J.R. Cuddell and F. Halzen, Phys.Lett. 175B:227 (1986).

G. VanDalen and A. Kernan, Phys. Rep. 106:297 (1984).

F. Herzog and Z. Kunszt, Phys. Lett. 157B:430 (1985).

K.G Wilson, Phys. Rev. D3:1818 (1973).

See for example J. Ellis, CERN-TH-4255/85 Lectures presented at the 28th Scottish Universities Summer School in Physics, Edinburgh. Scotland. or H. Haber and G. L. Kane Phys. Rep. 117:75 1985. 
71 E. Eichten and K. Lane, Phys. Lett. 90:125 (1980).

72

E. Farhi and L. Susskind, Phys. Rev. D20:3404 (1979).

73 R.D. Peccei, DESY 86-010 (1986).

74 G 'tHooft in Recent Developments in Field Theory, Ed G 'tHooft, Plenum (1980).

75 S. Coleman and B. Grossman, Nucl. Phys. B203:205 (1982).

76 W. Buchmuller, R. D. Peccei and T. Yanigida, Phys. Lett. 124B:67 (1983).

77 W. Buchmuller, R. D. Peccei and T. Yanigida, Nucl. Phys. B244:186 (1984).

78 L. Abbott and E. Farhi, Nucl. Phys. B189:547 (1981).

79 M. Claudson, E. Farhi and R. L. Jaffe, Phys. Rev. D34:877 (1986).

80 E. Eichten, K. D. Lane and M. Peshin, Phys. Rev. Lett. 50:811 (1982).

81 F. M. Renard, Phys. Lett. 116B:264 (1982).

82 E. Eichten, Lectures at the Yale University TASI summer school,Ed by F. Gursey, World Scientific Publishing Company (1986).

83 W. Bartel et al., Z. Physik C19:197 (1983)

84 W. Scott in Proc. of the 1986 International Conferance on High Energy Physics. Ed. S. Loken, World Scientific Publishing Company (1986).

85 G. Wolf, Lectures at this school. 
This report was done wilb suppon from the Department of Enerty: Any consiusions or opinions expresed in this report represent solkly those of the author(s) and not mocesurily those of The Regents of the Univerity of Californiv, the Lewrence Berteley Lnboratory or the Depertment of Energy.

Reference to a company or product name does not imply approval or recommendation of the product by the University of Californic or the U.S. Depanmens of Energy to the exclution of others that may be suilable. 